\title{
Using Machine Learning to Support Software Debugging
}

\author{
By \\ Xuetao Liu

\begin{abstract}
A thesis submitted to
The Faculty of Graduate Studies and Research

In partial fulfillment of the requirements for the degree of

Master of Applied Science
\end{abstract}

Ottawa-Carleton Institute for

Electrical and Computer Engineering

Department of Systems and Computer Engineering

Carleton University

Ottawa, Ontario, Canada

September 2007

Copyright $\odot 2007$ by Xuetao Liu 


$\begin{array}{ll}\begin{array}{l}\text { Library and } \\ \text { Archives Canada }\end{array} & \begin{array}{l}\text { Bibliothèque et } \\ \text { Archives Canada }\end{array} \\ \begin{array}{l}\text { Published Heritage } \\ \text { Branch }\end{array} & \begin{array}{l}\text { Direction du } \\ \text { Patrimoine de l'édition }\end{array} \\ \begin{array}{l}\text { 395 Wellington Street } \\ \text { Ottawa ON K1A ON4 }\end{array} & \begin{array}{l}\text { 395, rue Wellington } \\ \text { Ottawa ON K1A ON4 } \\ \text { Canada }\end{array}\end{array}$

Your file Votre référence ISBN: 978-0-494-33659-5 Our file Notre référence ISBN: 978-0-494-33659-5

NOTICE:

The author has granted a nonexclusive license allowing Library and Archives Canada to reproduce, publish, archive, preserve, conserve, communicate to the public by telecommunication or on the Internet, loan, distribute and sell theses worldwide, for commercial or noncommercial purposes, in microform, paper, electronic and/or any other formats.

The author retains copyright ownership and moral rights in this thesis. Neither the thesis nor substantial extracts from it may be printed or otherwise reproduced without the author's permission.
AVIS:

L'auteur a accordé une licence non exclusive permettant à la Bibliothèque et Archives Canada de reproduire, publier, archiver, sauvegarder, conserver, transmettre au public par télécommunication ou par l'Internet, prêter, distribuer et vendre des thèses partout dans le monde, à des fins commerciales ou autres, sur support microforme, papier, électronique et/ou autres formats.

L'auteur conserve la propriété du droit d'auteur et des droits moraux qui protège cette thèse. $\mathrm{Ni}$ la thèse ni des extraits substantiels de celle-ci ne doivent être imprimés ou autrement reproduits sans son autorisation.
In compliance with the Canadian

Privacy Act some supporting forms may have been removed from this thesis.

While these forms may be included in the document page count, their removal does not represent any loss of content from the thesis.
Conformément à la loi canadienne sur la protection de la vie privée, quelques formulaires secondaires ont été enlevés de cette thèse.

Bien que ces formulaires aient inclus dans la pagination, il n'y aura aucun contenu manquant.

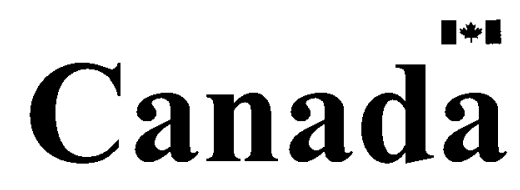




\begin{abstract}
Using a specific machine learning technique, this thesis proposes a method to identify suspicious statements during debugging which applies principles similar to Tarantula, an existing suspicious statement identification technique, but addresses its main flaw: its difficulty of dealing with the presence of multiple faults since it assumes that failing test cases execute the same fault(s). The improvement we present in this thesis is through the use of C4.5 decision trees to identify various failure conditions based on information regarding the test cases' inputs and outputs. Failing test cases executing under similar conditions are then assumed to fail due to the same fault. Statements are then considered suspicious if they are covered by a large proportion of failing test cases that execute under similar conditions. We report on two case studies that demonstrate improvement over the original Tarantula technique in terms of statement ranking. Another contribution of the thesis is to show that failure conditions as modeled by a C4.5 decision tree accurately predict failures and can therefore be used to help debugging.
\end{abstract}




\section{Acknowledgements}

I would like to thank my thesis supervisors Dr. Lionel Briand and Dr. Yvan Labiche for their guidance and financial support. They opened a door to me into the areas of software quality assurance and software quality management and gave me professional support, guidance and advice in the process of this research.

I would like to thank my wife Haizhen Mou, who gave me and continues to give me, such a great, active and meaningful life in Canada. Without her, I could not accomplish this thesis.

I would like to thank my parents, my brother and sister-in-law for their continuous and unconditional support through my life. I would also like to thank my parents-in-law and brother-in-law, who help me to deal with our errands.

I would like to thank everyone at the SQUALL lab for a great, friendly and supportive work environment.

I would like to express my appreciation to Dr. Zhiqiu Huang and his wife Professor Qing Li, Professor Zhangquan Lv, and my friends from EMSoftware lab of Nanjing University of Aeronautics and Astronautics, for their friendship and support in the past eight years.

I would also like to thank all the researchers who worked on and improved the Space program and artifacts over the years.

Xuetao Liu

August 30, 2007 


\section{Table of Contents}

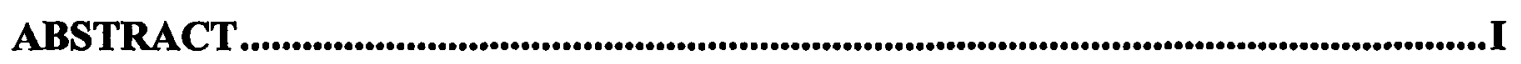

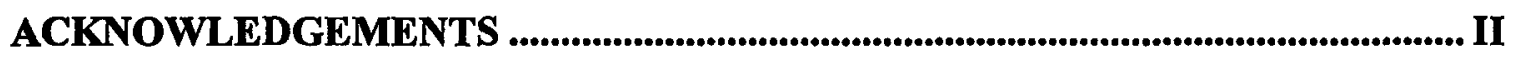

TABLE OF CONTENTS.......................................................................................III

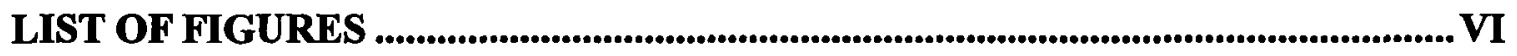

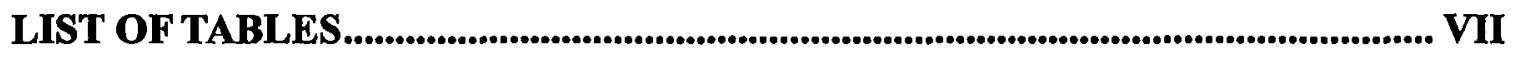

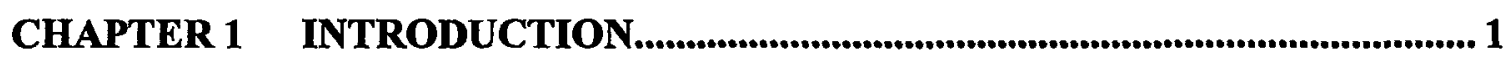

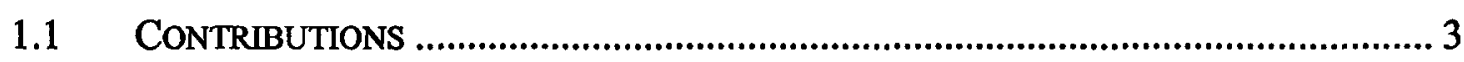

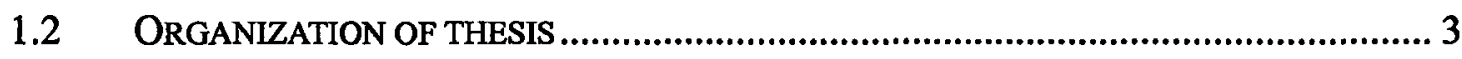

CHAPTER 2 RELATED WORK .................................................................. 4

2.1 AUTOMATED SOFTWARE DEBUGGING TECHNIQUES........................................... 4

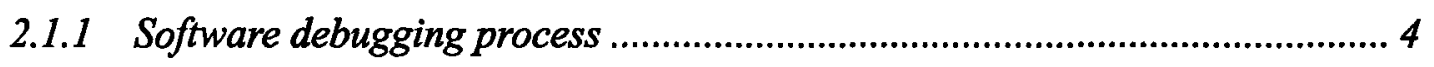

2.1.2 Manual software debugging strategies .......................................................6

2.1.3 Automated debugging techniques ............................................................ 7

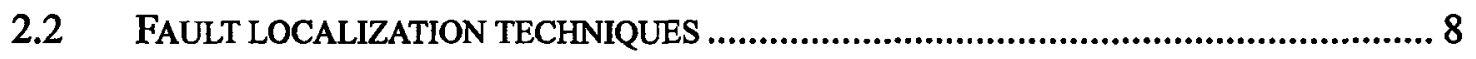

2.2.1 Fault localization based on anomaly...................................................... 8

2.2.2 Fault localization based on clusters ........................................................ 13

2.2.3 Diagnosis algorithm evaluation............................................................. 15

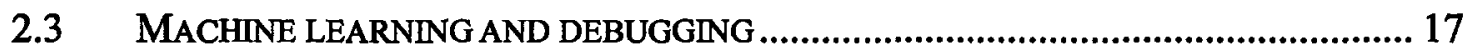

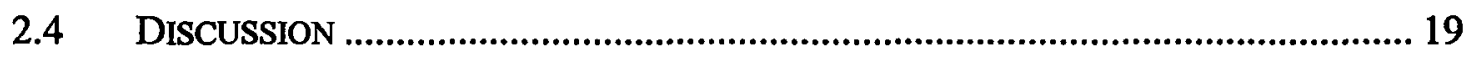

CHAPTER 3 BACKGROUND INFORMATION............................................ 20

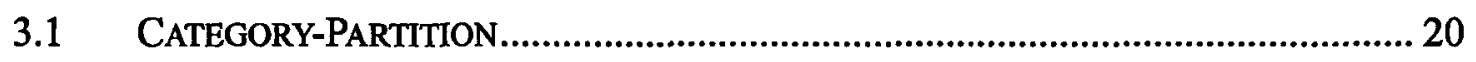

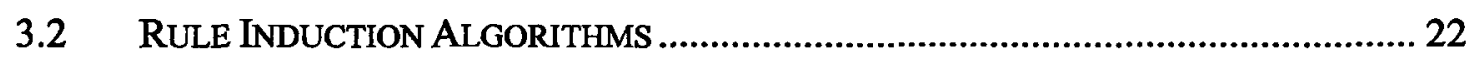

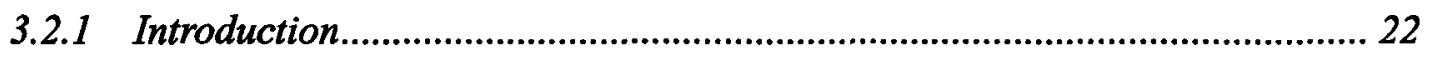

3.2.2 Model performance: Ten-fold cross-validation.......................................... 26

3.2.3 Model performance: cost of error prediction ........................................... 27

3.2.3.1 True positive rate and false positive rate.........................................................................................27

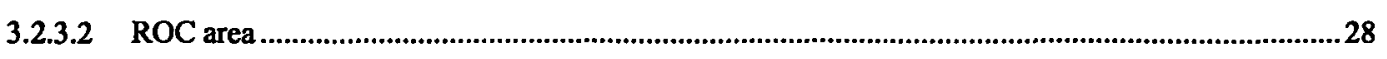

iii 


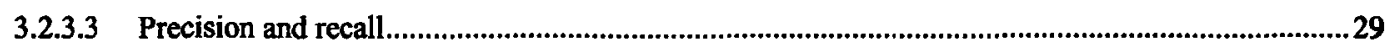

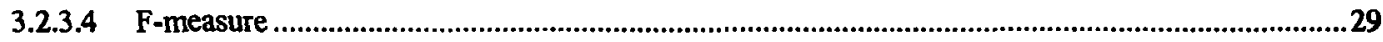

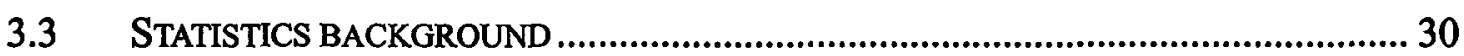

CHAPTER 4 STATEMENT RANKING STRATEGIES...................................... 31

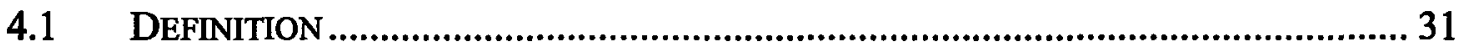

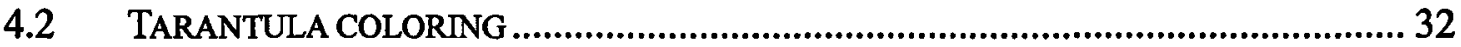

4.3 RANKING STATEMENTS BASED ON C4.5 RULES................................................ 34

4.3.1 Generating a C4.5 classification tree ..................................................... 34

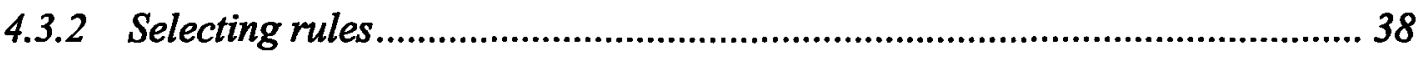

4.3.3 Computing a statement ranking ........................................................... 38

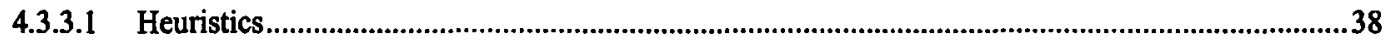

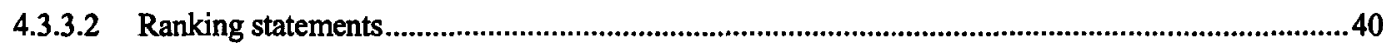

4.3.4 Iterative debugging process .................................................................... 42

4.4 MANUAL EFFORT AND AUTOMATION .............................................................. 44

CHAPTER 5 CASE STUDIES............................................................................. 46

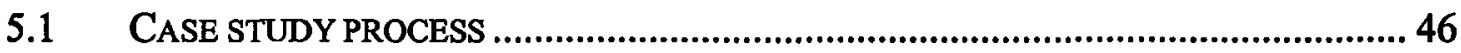

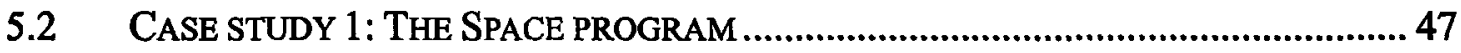

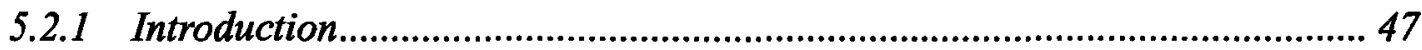

5.2.2 A multi-fault version of Space .................................................................. 49

5.2.3 Rule induction based on category partition.............................................. 50

5.2.4 Cost-Effectiveness analysis.................................................................. 52

5.2.4.1 Strategy one: fix all faults in the ranked set of statements ..........................................................53

5.2.4.2 Strategy two: fix faults in the first $20 \%$ of ranked statements........................................................58

5.2.5 Diagnosis accuracy evaluation.............................................................6 61

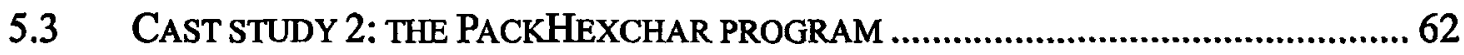

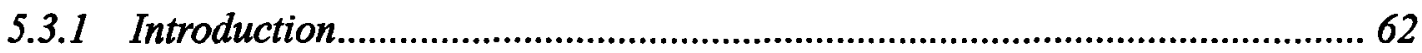

5.3.2 A multi-fault version of PackHexChar.......................................................6 63

5.3.3 Rule Induction Based on Category Partition............................................. 64

5.3.4 The statistical cost-effectiveness analysis ...................................................65 65

CHAPTER 6 CONCLUSIONS AND FUTURE WORK ...................................... 67

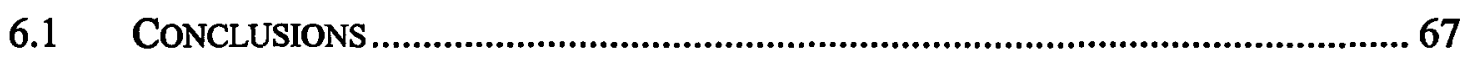

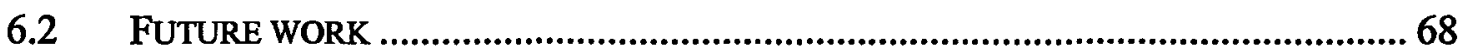

iv 


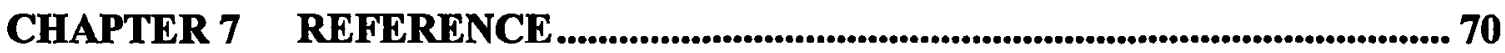

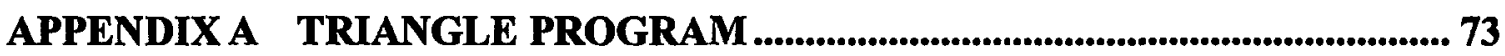

APPENDIX B CLASS DIAGRAM OF RUBAR ALGORITHM ......................... 75

APPENDIX C SPACE PROGRAM..................................................................... 76

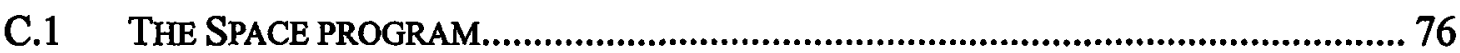

C.2 CATEGORY PARTITION DEFINITION............................................................... 76

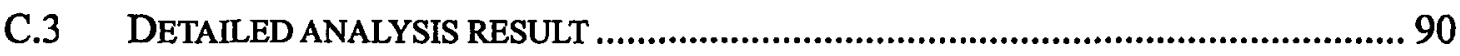

C.3.1 Strategy one: diagnose all statements executed..................................... 90

C.3.2 Strategy two: diagnose 20\% statements executed ................................. 91

APPENDIX D THE PACKHEXCHAR PROGRAM .......................................... 92

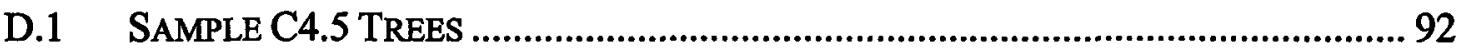

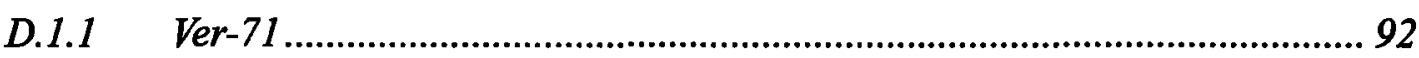

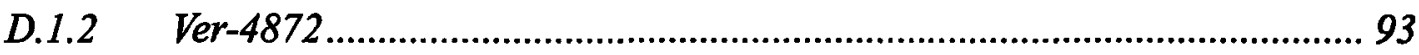

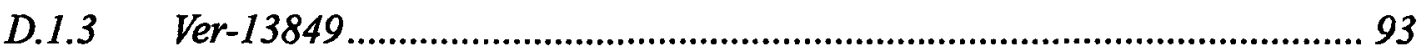




\section{List of Figures}

Figure 2-1 TRAFFIC: the seven steps in debugging ..........................................5

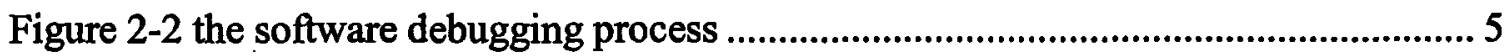

Figure 2-3 Set Union and Set Intersection......................................................... 10

Figure 2-4 Relationship between the size of DBBs and the diagnosis accuracy [3] ....... 17

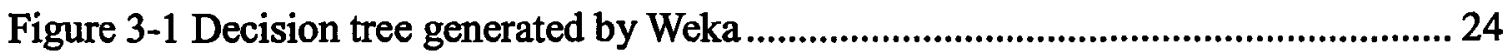

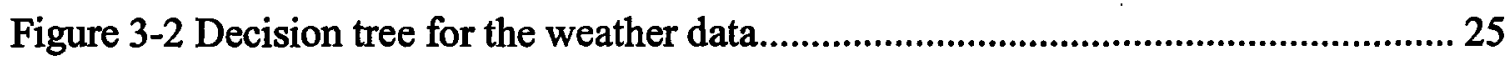

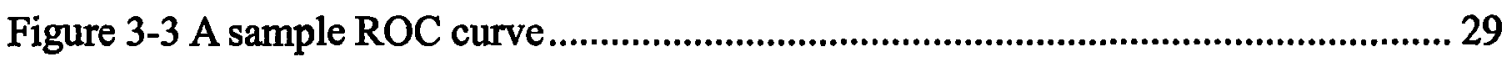

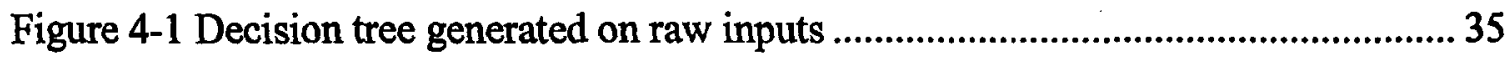

Figure 4-2 Decision tree of Triangle program generated by Weka ................................ 36

Figure 4-3 Decision tree generated on complete CP definition .................................... 36

Figure 4-4 Decision tree generated on non-complete CP definition............................... 37

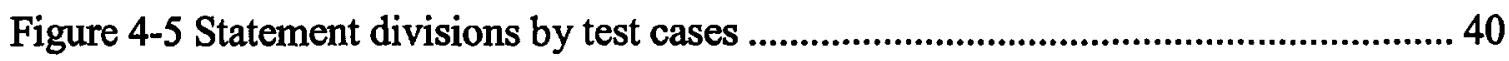

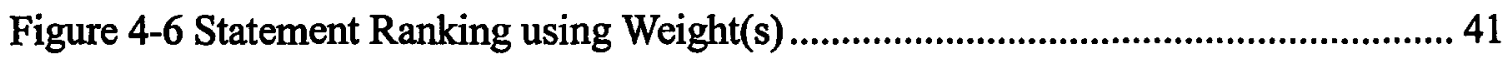

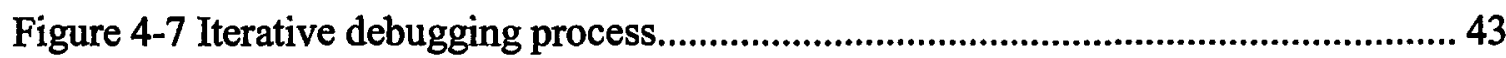

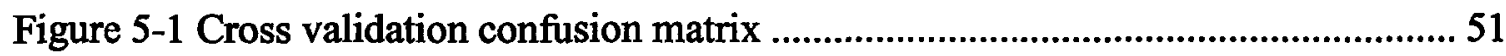

Figure 5-2 Fixing all the faults in the statement(s) executed..................................... 54

Figure 5-3 Execution sequence of faults: Bug16, Bug22 and Bug23 .......................... 56

Figure 5-4 Fixing faults in the first $20 \%$ of ranked statements.......................................59

Figure 5-5 Statistical statement ranking of 135 faulty versions .................................66

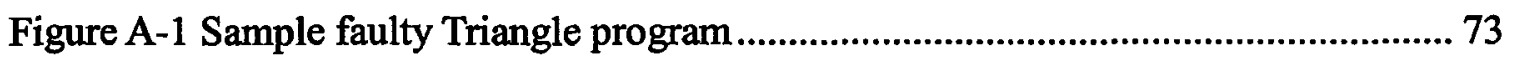

vi 


\section{List of Tables}

Table 3-1 A sample CP definition of the triangle program ........................................... 21

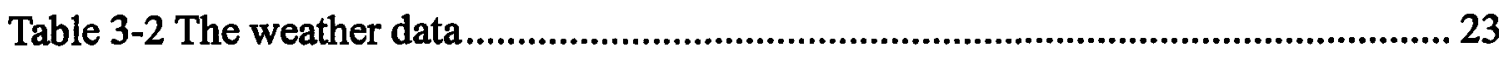

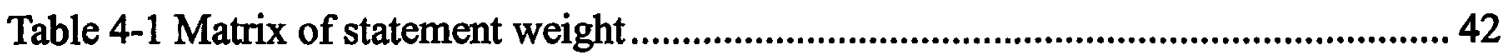

Table 5-1 Example Fail rules with accuracy and test case coverage .............................51

Table 5-2 Execution result of multi-fault version ................................................. 54

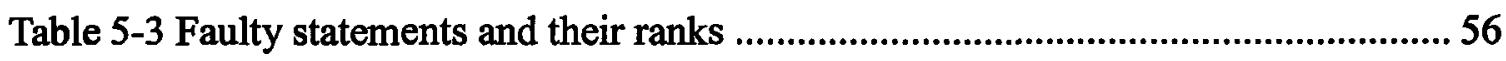

Table 5-4 Fixing faults in the first $20 \%$ of ranked statements ..................................... 58

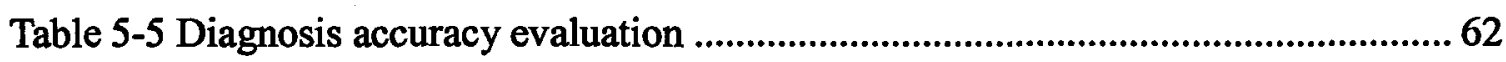

Table 5-6 Statistical accuracy of stratified 10-fold cross-validation by class.................. 65

Table A-1 A test suite and the test result of the triangle program ................................... 74

Table A-2 The test suite described by the CP definition .......................................... 74 


\section{CHAPTER 1 INTRODUCTION}

In the software life cycle, software testing is a critical element of software quality assurance [25]. In most cases, a software development group spends around 50 percent of total project effort on testing $[10,25]$. Software debugging is one of the most timeconsuming activities during software testing. When a test case uncovers an error, software debugging is the process that locates and removes the defect. Locating faults that cause failures is a very complex endeavor [37]. Though many techniques exist to support fault localization, this thesis is re-visiting the technique (Tarantula) that has shown to perform best on existing empirical studies [14]. Tarantula ranks statements by the degree of suspicion, which is determined by the proportion of the number of failing test cases that execute that statement over the number of passing test cases that execute that statement. Based on a careful analysis of Tarantula's cost-effectiveness, we identify a significant problem affecting its applicability and propose a solution to improve it based on the application of a machine learning technique (C4.5). At a high level, Tarantula uses the proportion of test cases that fail when executing a specific statement to determine the ranking of statements in terms of their likelihood to contain a fault. One important issue is that this method assumes that test cases fail due to the same fault, a situation that nearly never occurs in the presence of multiple faults. Another issue is that duplicated test cases would dramatically affect the ranking result generated by Tarantula.

We use the C4.5 decision tree algorithm [26] to analyze test executions and identify distinct conditions of failures in terms of properties on inputs and outputs. Note that, as further discussed below, a test specification is required (e.g., category partition [21]) as 
raw test case inputs are not helpful for machine learning algorithms to identify meaningful conditions of failures. Following a strategy similar to Tarantula, within each selected rule identified by $\mathrm{C} 4.5$, we analyze the statements executed by the test cases matching that rule and obtain a ranking of executed statements indicating the degree of suspicion of those statements. The rankings of all conditions are then combined into a single statement ranking. The most important difference with Tarantula is that because the statement coverage of test cases is analyzed within each distinct failure condition, these test cases are then more likely to fail due to the same faults, an assumption that does not hold when analyzing all test cases at once.

Note that, the failure conditions identified are of interest in their own right, independently of how they help statement ranking. We expect that programmers/testers would be helped a great deal if they are able to retrieve, from test case definitions and executions, precise conditions on inputs that are almost certain to trigger failures.

Through two case studies, we show that our solution has a practically significant, positive impact when compared to Tarantula. We also demonstrate that C4.5, following our procedure, can retrieve accurate rules predicting failures, thus implying that conditions triggering failures can be automatically and precisely characterized in terms of input and output properties.

The above approach can be applied in a number of contexts. For example, this might suit very well the test-driven development processes where large test suites are developed upfront and are available during development. It however requires that equivalence classes or categories/choices be defined to specify the test cases or at least - but this is less efficient-to re-express test cases at a high level of abstraction (test specification) in 
order to feed the machine learning algorithm. Our approach is probably more costeffective when test suites are specified by using a systematic, black-box technique.

\subsection{Contributions}

This thesis introduces a new strategy (RUBAR) to support software debugging by ranking the statements according to their degree of suspicion and by identifying the input conditions leading to failure. The contributions of this thesis include:

(1) Providing a fault-localization technique in a multi-fault environment;

(2) Combining the Category-Partition technique and machine learning technique to aid software debugging;

(3) Applying the C4.5 machine learning technique to find the conditions of failure, which can benefit software debugging directly or indirectly;

(4) Improving the Tarantula statement ranking technique by grouping test cases according to (similar) conditions of execution, and therefore according to the possible causes of failure;

(5) Empirical evaluation of our approach on two case studies.

\subsection{Organization of thesis}

The structure of this thesis is as follows. Chapter 2 discusses the related work. Chapter 3 presents a brief introduction on machine learning and the testing techniques used in this thesis. Chapter 4 revisits the Tarantula technique and presents the principles and rationale of our approach. Chapter 5 reports on our case studies and Chapter 6 presents conclusions and points out directions for future work. 


\section{CHAPTER 2 RELATED WORK}

A number of techniques exist to support the systematic debugging of software. The family of techniques directly relevant to our approach is used to detect anomalies, using Zeller's terminology [37], i.e., differences (e.g., in terms of coverage) between passing and failing executions of the program. These anomalies are good candidates for fault sites, hopefully helping to narrow down the amount of source code to investigate, and therefore reducing the time (and cost) required to locate faults.

\subsection{Automated software debugging techniques}

The objective of software debugging $[25,37]$ is realized by a combination of systematic evaluation, intuition and luck. As opposed to other activities in software testing that follows a set of precise rules, e.g., making test plans or designing test cases, the software debugging process relies on a programmer's experience, knowledge and intuition, which is hard to control and predict.

\subsubsection{Software debugging process}

Software debugging refers to the combined process of software diagnosis (fault localization), defect detection, and defect removal $[35,36]$. Software diagnosis is to find possible causes of failure; defect detection is to locate the exact statement(s), the root of failure; and defect removal is to fix the faulty statement(s). Zeller [37] decomposed software debugging process into seven steps named TRAFFIC (Figure 2-1). 


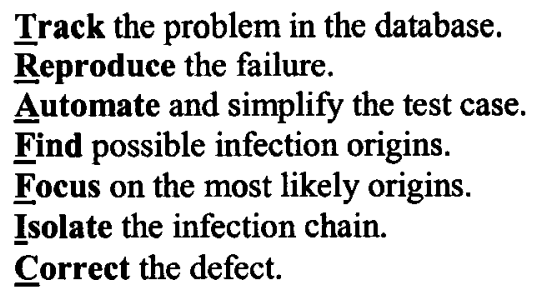

\section{Figure 2-1 TRAFFIC: the seven steps in debugging}

In general, software debugging begins with a failure of the program, which is detected by the execution of a test case(s) (Figure 2-2). After a set of time-consuming and tedious debugging activities, the debugging process will always end with one of the two outcomes [25]: (1) the cause of fault(s) is found and corrected, or (2) the cause is not found. In the latter case, the test suite is not abundant enough to help solve the problem. Therefore, the programmer (or tester) may suspect a cause, design new test case(s) to validate the suspicion, and work toward defect correction in an iterative process. In this thesis, we assume that the test suite has enough test cases to help detect and correct any fault the program has. Therefore, the problem of test suite improvement (e.g. $[3,5])$ is not in the scope of this thesis.

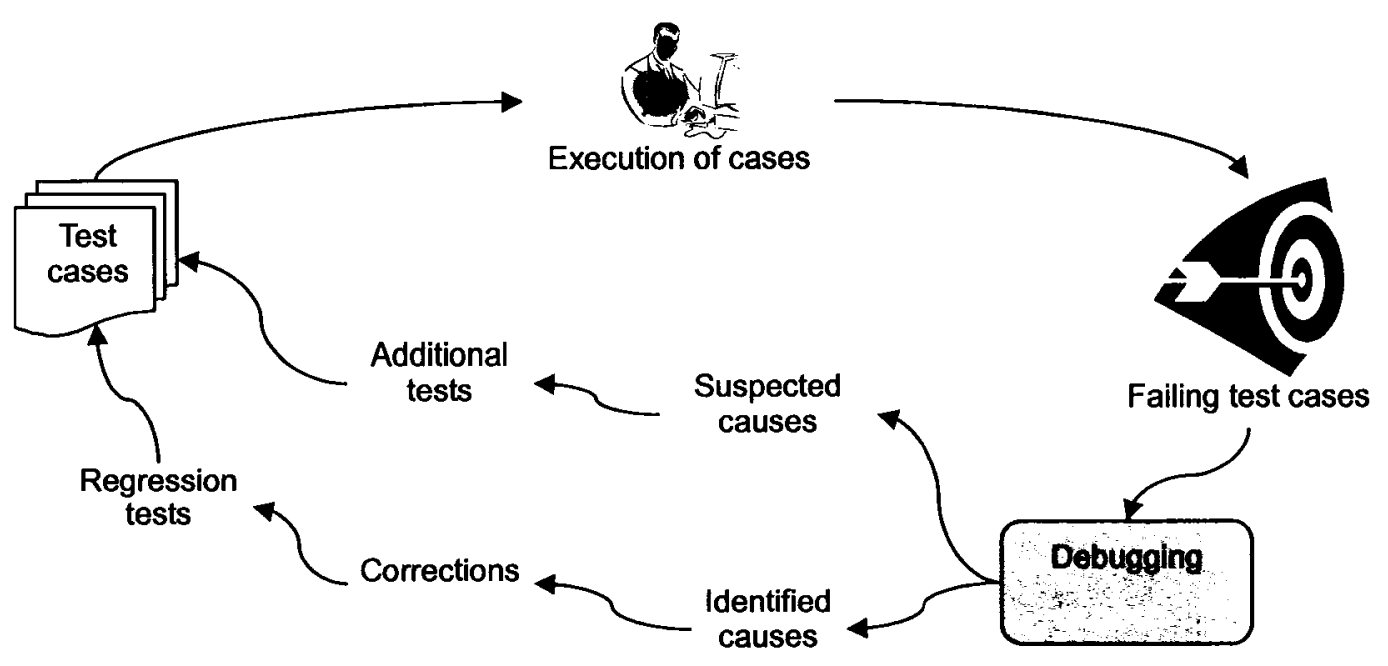

Figure 2-2 the software debugging process

Of the activities of software debugging, diagnosis (fault localization) represents $95 \%$ of 
the problem solving effort [20] and is the most important and difficult activity. This also makes the debugging process hard to predict and control. In principle, all faults can be solved manually [37]; but in practice, it is unwise to rely on manual debugging alone. There are some techniques (or strategies) [37] and tools (e.g. GDB, xSlice [1]) to aid software diagnosis and facilitate fault localization.

\subsubsection{Manual software debugging strategies}

Manual software debugging means that debugging tools provide no or little help during the process of software diagnosis. Manual software debugging is efficient only when the programmers are very familiar with the system under test or the program is very small. That is, sometimes programmers are able to guess the cause of a failure from the inputs and/or outputs of failed test cases. In this case, the programmers may like to diagnose the system manually. In general, there are three types of manual debugging strategies [20]:

- Brute force: probably the most common and least efficient method of isolating the cause of a software failure $[20,37]$. Brute force techniques are mainly used by debugging tools. The typical brute force techniques include memory dumps, run-time traces, break-points, studying execution states, and manually inserted assertions.

- Backtracking: a fairly common and efficient approach that can be used in small programs. Beginning at the point where a failure has been uncovered, the source code is traced backward (manually) until the site of the cause is found. It is obvious that, as the number of source lines increases, the number of potential backward paths may become unmanageably large.

- Cause elimination: This technique is based on induction and deduction and introduces 
the concepts of binary partitions. By cause elimination, a skilled programmer may find the defects without looking into the code. The data (input(s) and output(s) of the test cases) related to the failure occurrence are organized to isolate potential causes.

\subsubsection{Automated debugging techniques}

Since manual debugging is inefficient and time-consuming, there have been many studies on automated debugging techniques, to simplify debugging activities (especially the software diagnosis activity) or to guide the debugging process. In [37], Zeller classified the existing automated debugging techniques as:

- Simplified input: a debugging technique to identify duplicate problem reports and facilitate debugging. The basic idea of this technique is that the circumstances (inputs) not relevant for the problem can be omitted. After the simplification, a simple test case that contains only the relevant inputs can be generated. One of the input simplification techniques is the "divide-and-conquer" method introduced in [37], which uses the "binary tree" strategy to simplify the inputs. Once a test case fails, the inputs of the test case will be divided into two parts and two new test cases will be generated according to the input divisions. The program will be executed on both the new test cases. The inputs of the failed test case will be selected and divided again, until the smallest set of inputs causing the program to fail is obtained.

- Program slice: A program slice [31] is a portion of the program comprising of a set of statements which contribute, either directly or indirectly, to the value of certain variables at a certain point in the program. Program slices can be helpful in 
uncovering code smells ${ }^{1}$, especially in finding the common errors such as use of uninitialized variables, unused variables, or misused variables.

- Anomalies: In general, we can assume that a program works well most of the time. If a program fails nonetheless, we can use our knowledge about the passing runs and focus on the differences between the passing runs and the failing run. Such difference points out anomalies. Most of the automated software debugging techniques rely on anomalies to locate the cause of failures.

- Cause-effect chain: In [7], Clever and Zeller presented a state-based debugging technique named Cause Transition Technique. Instead of comparing the program state of a failing and a passing run, the technique makes hypotheses about how state changes will affect output. A cause transition is where a cause originates-that is, a cause transition points to program code that causes the transition and hence the failure.

In a realistic environment, these techniques can be combined to locate the $\operatorname{defect(s)}$ systematically. In this thesis, we introduce a new automated fault localization technique, which belongs to the anomalies category, which we further discuss next.

\subsection{Fault localization techniques}

\subsubsection{Fault localization based on anomaly}

Set Union and Set Intersection [27] are the simplest techniques to detect anomalies, which use the difference(s) among the failing executions and the passing executions to find suspicious statements. In 1982, Weiser [32] originally presented the idea of using

\footnotetext{
${ }^{1}$ A code smell is a hint that something may be wrong in the code. It usually corresponds to common design errors or implementation problems in the program.
} 
static program slices to locate bugs. The basic idea of this theory is that the cause of a failure is more likely to be in the static program slice(s) of the site or variable(s) where the failure is recovered than other program. Agrawal etc [1] presented a technique to help fault localization by computing the difference of the statements executed by two test cases-one passing test case and one failing test case. A faulty statement is more likely to present in the set of statements that are executed by the failing test case and are not executed by the passing test case. In [22], Pan and Spafford presented a set of heuristics that use set algebra of test cases' dynamic slice to reduce the search scope of fault localization. Each heuristic will suggest a set of suspicious statements whose size is usually smaller than the size of the executed statements.

The basic idea of the set union and set intersection technique is that failing and passing executions (likely) involve different statements. It assumes that the faults reside in the statements executed by a failing test case and do not exist in the statements executed by a passing test case. One can therefore look at the statements executed in a failing execution but not in passing executions to locate fault (Set Union-set of statements executed in a failing execution minus the union of the sets of statements executed in passing executions, represented by the shadow part in Figure 2-3(a)). Alternatively, one can ignore the statements common to passing executions but absent from a failing execution (Set Intersection-intersection of the sets of statements executed in passing executions minus the set of statements executed in a failing execution, represented by the shadow part in Figure 2-3(b)). 


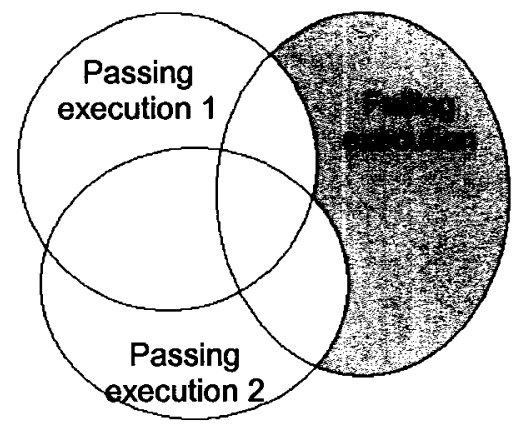

(a) Set Union, features unique to the faulty run

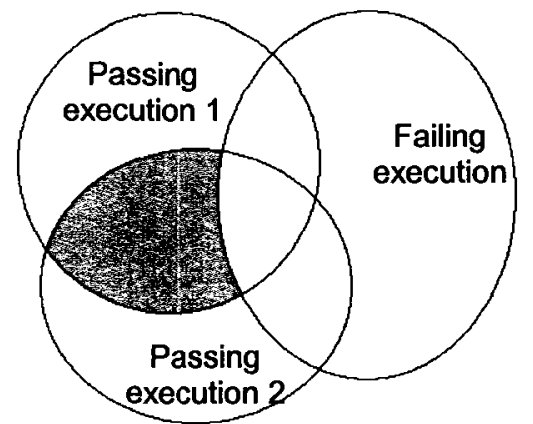

(b) Set Intersection, features common to the success runs

Figure 2-3 Set Union and Set Intersection

Instead of using all the passing runs to perform the statements separation, Renieris and Reiss [27] selectively choose a single best passing run for the set difference operation. The passing run is selected by a Nearest Neighbor algorithm, which consists in finding a single passing execution that is as similar as possible (e.g., in terms of code covered) to a failing execution (i.e., the nearest neighbor). The difference between the two executions is then worth investigating when looking for faulty statements.

In [9], the authors recognize that some failures are due to specific sequences of method calls, rather than simply the coverage of some statements. Their approach is specific to Object-Oriented systems (they work at the class level), and, in particular, Java. The approach is to collect, for each class of the system, the sequences of calls that the instances of the class execute at runtime. The idea is then to compare, for each class, the sequences triggered by a failing execution and the sequences triggered by the passing executions, and then rank the classes in such a way that the classes whose sequence sets differ the most get the higher priority. (See [9] for the precise ranking mechanism.)

These researchers tend to compare one failing execution (instead of several failing ones) against a number of passing ones, assuming different failing executions may be caused by 
different faults, which is something unknown beforehand. On the contrary, Jones et al. [15] consider all executions together, recognizing that the more a statement is executed by failing executions, the more likely the statement is a faulty statement. The Tarantula technique associates a "color" (ranking) to each statement, accounting for all executions: the redder the statement, the higher the percentage of failing test cases that execute this statement. The statement color can be used to rank the statements, as suggested in [14], in order to support the search for faults during debugging.

A similar technique is discussed in [17]. It is limited to the observation of certain predicates during program execution (e.g., the value of a predicate in an if-statement) as the authors collect execution data from deployed programs, and instrumentation is therefore limited to ensure a reduced impact on the user. The result is a rank of monitored predicates indicating the (statistical) likelihood of the predicate being a failing condition as well as being the fault location. This is expanded upon in [19] where the authors define a similarity function between predicate rankings to group executions that (likely) fail due to the same fault.

As opposed to Tarantula and other ranking techniques, the Set Union, Set Intersection, and Nearest Neighbor techniques simply identify an initial set of suspicious statements to start the search from, not a ranking of statements. As suggested in [14], they can however be augmented to produce a ranking of statements, as originally suggested in [27] for the Nearest Neighbor technique. The ranking is based on a breadth-first search (backward and forward directions) in the system dependence graph from the initial statements. Nodes at the same distance from initial statements are given the same rank.

A number of experimental results discuss how these techniques compare to one another. 
First, since the technique in [9], based on method sequences, ranks classes instead of statements, it cannot be directly compared with the other three techniques. The Nearest Neighbor technique has been shown to outperform the Set Union and Set Intersection techniques [27]. Tarantula has been compared to Set Union, Set Intersection and Nearest Neighbor [14]. For comparison purposes, the authors used the so-called Siemens suite of seven different programs [12]: 122 faulty versions (one fault per version) were used. The ranking of each technique was used to identify the rank of the (known) faulty statement of each faulty version. This rank was used to compute a score for each faulty version, corresponding to the percentage of the program (code) that does not need to be examined to find the faulty statement. They then compare the cumulative number of faulty versions, on the Y-axis, as the score goes from $\mathbf{9 9 \%}$ (the technique pinpoints the faulty statement) to $0 \%$ (all the code has to be verified to find the faulty statement), on the $\mathrm{X}$-axis. Results show that Tarantula outperforms the other three techniques. In particular, in $55.7 \%$ of the faulty versions, the fault was found by examining less than $10 \%$ of the code. These results were the main motivation for our research to use Tarantula as a basis of comparison to improve upon.

The effectiveness of Tarantula was originally reported in [15] on 20 faulty versions (one fault per version) of the Space program [30]. No ranking was used though, and the authors only studied the coloring of faulty and non-faulty statements, showing that the former were always colored in red (i.e., suspicious) whereas the latter were most of the time colored in green. (A large number of the non-faulty statements received a reddish or yellowish color though.) There were two notable exceptions: for two faulty versions, the faulty statements received an average color (yellow) - they would therefore not 
necessarily appear suspicious to the tester-because they were executed by all or most of the test cases. These statements initialize the global variables used by all test case executions. The authors also studied Tarantula on multiple-faults versions. They observed that the effectiveness of Tarantula declines (fewer faulty statements are colored red) as the number of faults increases, though no precise trend can be established.

From the discussion above, we can see that SET operations are the basis of the anomaly debugging techniques. Except for the Nearest-Neighbor algorithm, none of other methods account for the fact that to compare executions and identify suspicious statements, the selected passing executions must be similar to the failing execution. That is, the selected test cases (failing and passing) need to test the same or similar functions. It is only when the failing run and passing runs are similar that the differences between the statements executed by the failing test case and the passing test cases are meaningful.

There are other debugging techniques that are not discussed here, as they are less relevant to our context. These include deduction techniques (e.g., program slicing, delta debugging), and observation techniques (e.g., states, invariants). The interested reader is referenced to [37] for further details.

\subsubsection{Fault localization based on clusters}

Cluster-based fault localization methods $[13,38]$ are related to our method directly. This type of method is usually applied in a multi-fault environment, which is more realistic. In a multi-fault environment, the failure of test cases could be caused by different defects. This makes the fault localization more complex than a single fault version does. The fault-localization methods introduced in section 2.1 can not be simply applied in the 
multi-fault environment. Otherwise, the effectiveness of fault localization will degrade, as James et al. discovered [15].

Since test cases fail due to different faults in a multi-fault environment, it is reasonable to group the failed test cases by the causes of the failure before a diagnosis algorithm is applied for fault localization. That is, the set of failed test cases are partitioned into clusters that target different faults. Then a fault localization method $[1,7,15,27]$ is employed to locate the faulty statement(s) for each cluster. For example, James et al. [13] employ the agglomerative hierarchical clustering method [4] to group failed test cases and use the Tarantula coloring method to refine the clusters generated and perform the fault localization. The clustering algorithm uses behavior models, generated from execution data, to group the failed test cases. The second clustering method James et al. [13] presented is based on statement ranking generated by a fault-localization algorithm. The technique is to first compute the fault-localization suspiciousness rankings of statements using all the failed test cases (together with all the passed ones), and to use the Jaccard similarity metric to compute the pairwise similarities among these rankings. Then the technique clusters statements by taking a closure of the pairs that are marked as similar.

The effectiveness of cluster-based fault localization is evaluated by James et al. [13]. They created 100 8-fault versions of the Space program by choosing from the available faults at random. For the two clustering scenarios [13], the mean numbers of statements to be examined before a real fault is localized are observed to be 31.5 and 26.43 respectively. 


\subsubsection{Diagnosis algorithm evaluation}

Diagnosis algorithms aim at isolating a minimal subset of statements which are highly suspected to cause the failure. The smaller the subset of statements containing the real fault, the more accurate a diagnosis algorithm is. Baudry, et al. [3] define two criteria to evaluate the accuracy of a diagnosis algorithm:

Diagnosis accuracy: the average number of statements to be examined before finding the actual faulty statement;

Relative diagnosis accuracy: the corresponding percentage of the source code of the program to examine to find the actual faulty statement.

Baudry, et al. [3] also identified the main attribute of a program that reduces the diagnosis analysis effort. This attribute is called DBB: Dymamic Basic Block; and depends on both the test traces and the code structure. A dynamic basic block [3] is a group of statements that are executed by the same set of test cases. From the definition, we can draw out that the statements in a single DBB are indistinguishable [3] and will obtain the same rank in the ranking generated by a diagnosis algorithm. The authors then define an optimal diagnosis algorithm as one that always selects the dynamic basic block containing the actual faulty statement(s) as the most suspicious.

An ideal accuracy of diagnosis can be defined under the following assumptions. Assuming that faults are uniformly distributed; each statement has the same probability to be faulty. Assuming the diagnosis algorithm is optimal, and then the ideal accuracy of diagnosis can be defined as:

$$
\operatorname{IdealAccuray}(P)=\sum_{b \in D B B s} p(b) \times n(b)=\sum_{b \in D B B s} \frac{|b|^{2}}{2 \times|S|}
$$


where:

$D B B$ is the set of $D B B$ identified by a diagnosis algorithm;

$p(b)$ is the probability that the fault is located in $b \in D B B s$ and $p(b)=|b||S|$, suppose that faults are uniformly distributed and $S$ is the set of executable statements of the program.

$n(b)$ is the average amount of code to examine in $a B B$ b and $n(b)=|b| / 2$.

Through the ideal accuracy of diagnosis, Baudry et. al. [3] concluded that the size of dynamic basic block is the main attribute which impacts the diagnosis accuracy. They performed their case study on a BOOK system and validated the relationship between the diagnosis accuracy and dynamic basic blocks. The result [3] of the case study is presented as Figure 2-4. We can see that the smaller the size of dynamic basic blocks, the more accurate a diagnosis algorithm [3]. 

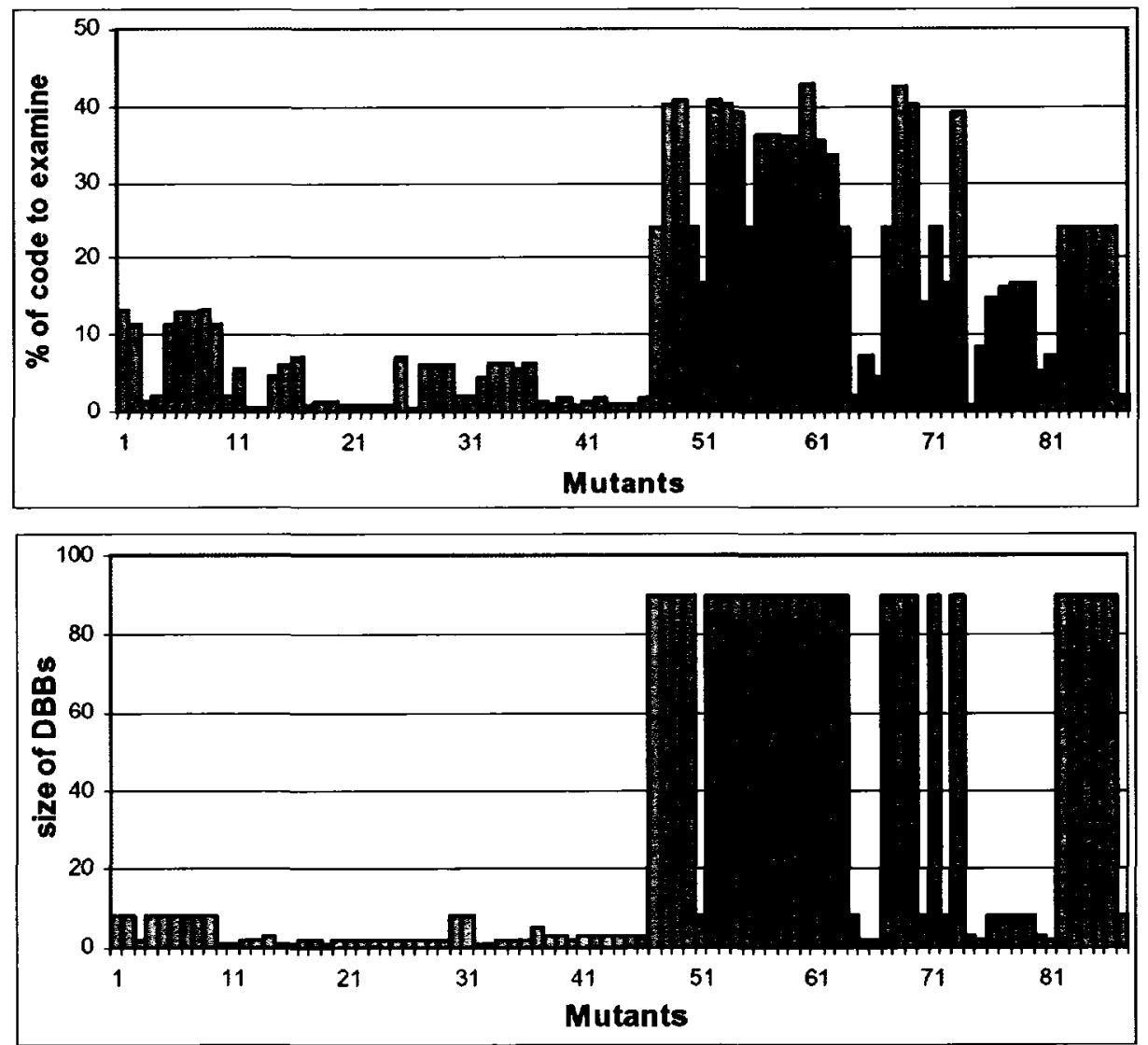

Figure 2-4 Relationship between the size of DBBs and the diagnosis accuracy [3]

There are, of course, other ways once can approach the comparison of these techniques. For example, in [14], James et al. evaluated the efficiency of the techniques by recording the amount of time required to use them.

\subsection{Machine learning and debugging}

Another, more recent, body of work related to our problem, attempts to use data mining techniques to help identify suspicious code. In [18], the authors record passing and failing program executions, monitoring true and false evaluations of predicates (e.g., if statements). They developed a data mining technique that relies on distributions of true and false evaluations in failing and passing executions to rank (suspicious) functions. They limit their analysis to the statements containing predicates, report good results 
(buggy functions are ranked first) on the Siemens program suite (each faulty version contains only one fault, though), but do not present any comparison with other techniques. Similarly, Podgurski et al. [24] use passing and failing execution profiles to help debugging. The execution profiles entail recorded information that is not limited to predicates but can contain any data that can be collected about program executions (e.g., a program variable value, a call stack). The program execution features in the profile that can best distinguish passing from failing executions are first identified using logistic regression. Because the level of abstraction of the collected data needs to be increased to help predict failure, failing profiles are then grouped using either a cluster analysis technique or a multivariate visualization technique. Those groups are then analyzed by the tester to understand failing execution conditions. The type of properties that can be uncovered using this approach is limited and remains to be investigated.

A machine learning technique is used in [6] to identify general program properties (e.g., variables not initialized) that likely indicate the presence of faults in programs. As opposed to the above-mentioned approaches, it does not require test case executions as it only relies on program analysis (though, for evaluation purposes, the authors use "likely invariants" [11] as program properties, whose discovery requires program executions). For a scalar variable $x$, examples of properties are: equality to a constant $(x=a)$, and lying in a range (e.g., $a<x \leq b)$. In the learning step, properties of correct and incorrect programs are used to build a learning model (e.g., Support Vector Machine [33]) to distinguish faulty and non-faulty programs). In the classification step, a new program's properties are used as inputs and those properties are ranked according to the strength of their association with faulty programs. It should be noted that the approach above requires that 
abstract properties be defined beforehand, which is similar to our use of categories and choices.

\subsection{Discussion}

What we propose in this research is different from the work presented above in the following ways: (1) We reuse black-box testing specifications (under the form of categories and choices) as inputs to identify failure conditions; (2) Failure conditions are formalized as logical rules, so as to provide interpretable inputs to support debugging; (3) These rules are generated by using a machine learning algorithm to automatically generate interpretable rules from transformed test cases; (4) Those rules are then applied to rank statements according to their likelihood of containing a fault; (5) The test cases are clustered according to the test case definitions (abstract test data), instead of the execution data.

One motivation of our research is to rely on inputs that should anyway be part of any test plan and is therefore of general usefulness to the tester beyond debugging. A second motivation is to provide feedback to testers in a form that can be used to better understand the reasons for failure (by the classification rules). Third, we want our technique to handle the common case where multiple faults are present in the program and different executions fail due to different faults. 


\section{CHAPTER 3 BACKGROUND INFORMATION}

This chapter presents a background of materials that are needed to fully understand this thesis. Section 3.1 introduces the Category-Partition (CP) black box testing technique. Section 3.2 discusses rule induction algorithms and explains the choice of $\mathrm{C} 4.5$ decision trees.

\subsection{Category-Partition}

The Category Partition technique [21] is one of the most well-known black-box techniques. Compared to equivalence class partitioning (in which only partitions input value domains) and other test methods, it is more general and encompassing. The CP method seeks to generate test cases that cover functions. To apply the CP method, one identifies the parameters of each function, the characteristics (categories) of each parameter and the choices of each category. Categories are properties of parameters that can have an influence on the behavior of the software under test (e.g., size of an array in a sorting algorithm). Choices (e.g., whether an array is empty) are the potential value of a category. Test frames (abstract test case) and test data are generated according to the categories and choices defined.

When applying the CP methodology, one is also supposed to define constraints and interdependencies between choices across categories. Because this is not required in our context, we will not refer to this aspect of the CP methodology.

Let's take a simple but hopefully illustrative example: for the Triangle program [16], the input values characterize the length of triangle sides and the output is the type of the 
triangle determined by the three sides (Equilateral, Isosceles, Scalene, or NotATriangle). When applying the CP method we define categories and choices from the features (not from the boundary of the input values for a parameter) of the triangle program. These categories and choices describe the character of the length of the triangle sides and the relationship among them.

Table 3-1 is the category-partition definition of the Triangle program. For instance, the category compare $\left(s_{1}, s_{2}\right)$ defines the relationship between the length of sides $s_{l}$ and $s_{2}$, which is further described by choices: $\mathbf{s}_{1}<\mathbf{s}_{2}, \mathbf{s}_{1}=\mathbf{s}_{2}$, and $\mathbf{s}_{1}>\mathbf{s}_{2}$. The categories CA04 to CA10 in Table 3-1 define the criteria for three values to determine a triangle. Note that we did not define constraints as we discussed above. For more information about the application of constraints, please refer to [21].

Table 3-1 A sample CP definition of the triangle program

\begin{tabular}{|c|c|c|c|c|c|}
\hline & Categories & Choices & & Categories & Choices \\
\hline CA01 & \multicolumn{2}{|c|}{$\operatorname{compare}\left(\mathrm{s}_{1}, \mathrm{~s}_{2}\right)$} & CA06 & \multicolumn{2}{|l|}{$\mathrm{s}_{2}<\mathrm{s}_{1}+\mathrm{s}_{3}$} \\
\hline & CH0101 & $\mathrm{s}_{1}<\mathrm{s}_{2}$ & & CH0601 & Yes \\
\hline & CH0102 & $\mathrm{s}_{1}-\mathrm{s}_{2}$ & & $\mathrm{CH} 0602$ & No \\
\hline & CH0103 & $s_{1}>s_{2}$ & CA07 & \multicolumn{2}{|l|}{$\mathrm{s}_{3}<\mathrm{s}_{1}+\mathrm{s}_{2}$} \\
\hline $\mathrm{CA02}$ & \multicolumn{2}{|c|}{$\operatorname{compare}\left(\mathrm{s}_{2}, \mathrm{~s}_{3}\right)$} & & CH0701 & Yes \\
\hline & CH0201 & $s_{2}<s_{3}$ & & CH0702 & No \\
\hline & $\mathrm{CH} 0202$ & $\mathrm{~s}_{2} \rightarrow \mathrm{s}_{3}$ & CA08 & \multicolumn{2}{|l|}{$s_{1}>\left|s_{2}-s_{3}\right|$} \\
\hline & $\mathrm{CH} 0203$ & $\mathrm{~s}_{2}>\mathrm{s}_{3}$ & & CH0801 & Yes \\
\hline CA03 & \multicolumn{2}{|c|}{ compare $\left(\mathrm{s}_{3}, \mathrm{~s}_{1}\right)$} & & $\mathrm{CH} 0802$ & No \\
\hline & CH0301 & $s_{3}<s_{1}$ & CA09 & \multicolumn{2}{|l|}{$\mathrm{s}_{2}>\left|\mathrm{s}_{1}-\mathrm{s}_{3}\right|$} \\
\hline & CH0302 & $s_{3}=s_{1}$ & & CH0901 & Yes \\
\hline & CH0303 & $s_{3}>s_{1}$ & & $\mathrm{CH} 0902$ & No \\
\hline CA04 & \multicolumn{2}{|c|}{ and $\left(s_{1}>0, s_{2}>0, s_{3}>0\right)$} & CA10 & \multicolumn{2}{|l|}{$s_{3}>\left|s_{1}-s_{2}\right|$} \\
\hline & $\mathrm{CH} 0401$ & Yes & & $\mathrm{CH} 1001$ & Yes \\
\hline & CH0402 & No & & \multirow[t]{4}{*}{ CH1002 } & No \\
\hline CA05 & \multicolumn{2}{|l|}{$\mathrm{s}_{1}<\mathrm{s}_{2}+\mathrm{s}_{3}$} & & & \\
\hline & CH0501 & Yes & & & \\
\hline & $\mathrm{CH} 0502$ & No & & & \\
\hline
\end{tabular}




\subsection{Rule Induction Algorithms}

\subsubsection{Introduction}

There is a large number of machine learning and data mining techniques [33]. They differ widely in terms of their basic principles, their working assumptions, and their weaknesses and strengths. None of the techniques is inherently better than the other and which one is most appropriate tends to be context dependent.

A specific category of machine learning techniques focuses on classification, which is the problem at hand in this project as we want to explain and predict when test cases fail. These techniques will generate classification rules [33]. Examples of such techniques include the C4.5 decision tree algorithm [26] or the Ripper rule induction algorithm [8]. In our context, the rules would look like conditions on the test inputs and the outputs being associated with probabilities of failures (Fail/Pass classification). The main advantage of these techniques is the interpretability of their models: certain conditions imply a certain probability of failure.

Some classification techniques, like C4.5, partition the data set (e.g., the set of test cases) in a stepwise manner using complex algorithms and heuristics to avoid over-fitting the data with the goal of generating models that are as simple as possible. Others, like Ripper, are so-called covering algorithms [8] and generate rules in a stepwise manner, removing observations that are "covered" by the rule at each step so that the next step works on a reduced set of observations. With coverage algorithms, rules are interdependent in the sense that they form a "decision list" where rules are supposed to be applicable in the order they were generated. Because this makes their interpretation more difficult, we will 
use a classification tree algorithm, namely C4.5, and use the WEKA tool [33] to build and assess the trees.

As an example, consider the weather problem [33], which is a simple dataset used by Witten et. al. to illustrate machine learning methods. It concerns the conditions (attributes) that are suitable for playing some unspecified game. There are totally four attributes: outlook, temperature, humidity, and windy. The outcome is whether to play or not. A sample with 14 instances is given out in Table 3-2. The weather problem is a typical twocall prediction problem, which has two possible results: yes or no.

\section{Table 3-2 The weather data}

\begin{tabular}{|l|l|l|l|l|}
\hline \multicolumn{1}{|c|}{ Outlook } & Temperature & \multicolumn{1}{c|}{ Humidity } & \multicolumn{1}{c|}{ Windy } & \multicolumn{1}{c|}{ Play } \\
\hline sunny & hot & high & false & no \\
\hline sunny & hot & high & true & no \\
\hline overcast & hot & high & false & yes \\
\hline rainy & mild & high & false & yes \\
\hline rainy & cool & normal & false & yes \\
\hline rainy & cool & normal & true & no \\
\hline overcast & Cool & normal & true & yes \\
\hline sunny & Mild & high & false & no \\
\hline sunny & cool & normal & false & yes \\
\hline rainy & mild & normal & false & yes \\
\hline sunny & mild & normal & true & yes \\
\hline Overcast & mild & high & true & yes \\
\hline Overcast & hot & normal & false & yes \\
\hline Rainy & mild & high & true & no \\
\hline
\end{tabular}

Figure $3-1$ is the C4.5 classification tree generated by Weka (the J48 algorithm). To generate this tree, we use the default values for all the parameters of the algorithm. The classification tree in Figure 3-1 has five leaves and eight nodes. Line four to line ten describe the structure of the tree. The content of each line is defined using the following grammar:

$$
<\text { rule }>\rightarrow<\text { rootLine }>\{<\text { nodeLine }>\}^{*}<\text { leafLine }>\mid<\text { leafLine }>
$$


$<$ rootLine $>\rightarrow<$ category $>=<$ choice $>$

$<$ nodeLine $>\rightarrow\{\mid\}^{?}<$ category $>=<$ choice $>$

$<$ leafLine $>\rightarrow\{\mid\}^{*}<$ category $>=<$ choice $>[:<$ predicatedResult $>$ (total-

instances [/misclassified-instances]])

where total-instances is the number of instances whose inputs satisfy the conditions of a rule;

misclassified-instances is the number of instances whose inputs satisfy the conditions of a rule, but the output does not fit the predicted output of the rule. It is obvious that the misclassified-instances shall be less than the number of correctlyclassified instances.

From Figure 3-1, we can see that the rules generated are accurate because there is no misclassified instance: as per the above grammar, misclassifications would appear in parentheses after the " $/$ ' character, which is not the case in Figure 3-1. We can also find that it is very fast to generate such a tree (the time taken to build this tree is zero second).

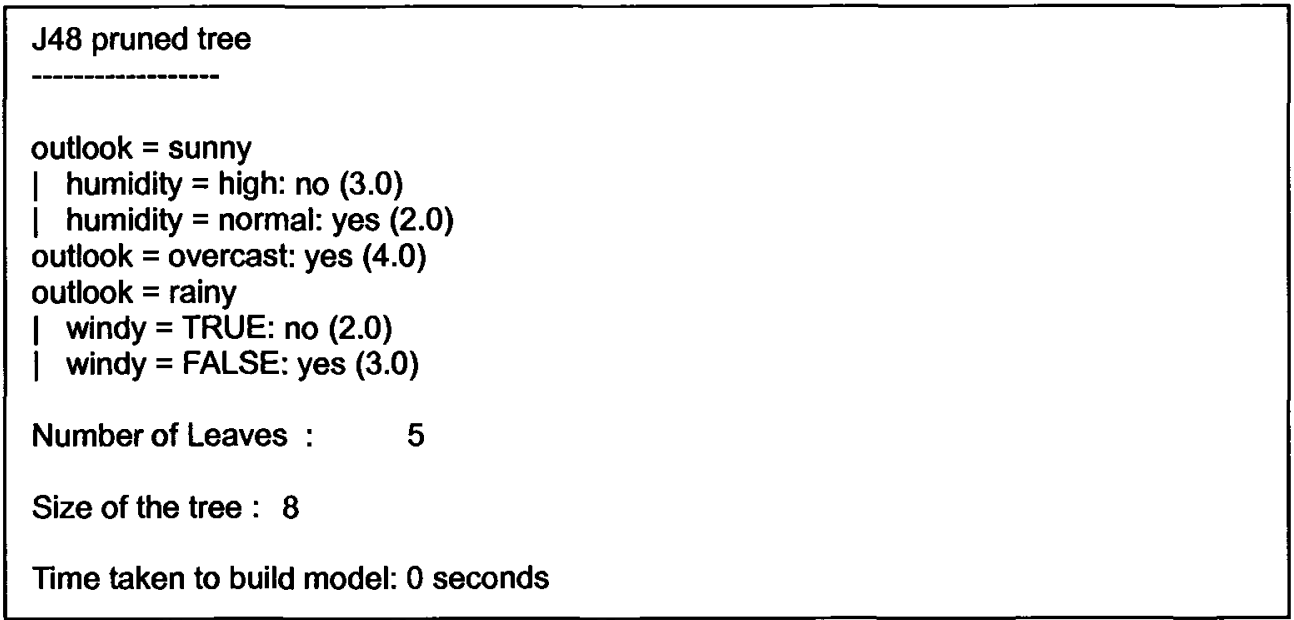

Figure 3-1 Decision tree generated by Weka

Figure 3-2 is a graphic view of the C4.5 tree of Figure 3-1. Each node represents a (sub)set of instances and is characterized by a number of conditions on input or output properties. Some nodes are terminal (e.g., node 5) whereas others are further decomposed 
(e.g., node 4). A terminal node has a class label instead of an attribute; this is the classification and is followed by the number of instances that reach that leaf. The class label of a terminal node is determined by the class of the majority instances classified by the corresponding path. If there were incorrectly classified instances reaching a leaf node, the number would be expressed as total-instances/misclassified-instances. Our goal when building the tree is to obtain leaves that are as consistent as possible, i.e., which contain instances that are either mostly yes or no. This is one of the objectives of the C4.5 algorithm. Edges represent values for an attribute, and a path from the root node of the tree to any leaf can be considered a rule characterizing play or not, i.e., a conjunction of attribute values.

In Figure 3-2, nodes are the attributes of the weather problem and leaves are labeled as "yes" or "no", which stand for "play" and "no play". Node (5), for example, captures all instances with "sunny" outlook and "high" humidity. It is a terminal node predicting "do not play" (labeled as "no"): in this leaf, the number of classified (resp. misclassified) instances is three (resp. zero). Figure 3-2 highlights a rule for one of the paths where outlook = sunny AND humidity = high predicts no (don't play). 


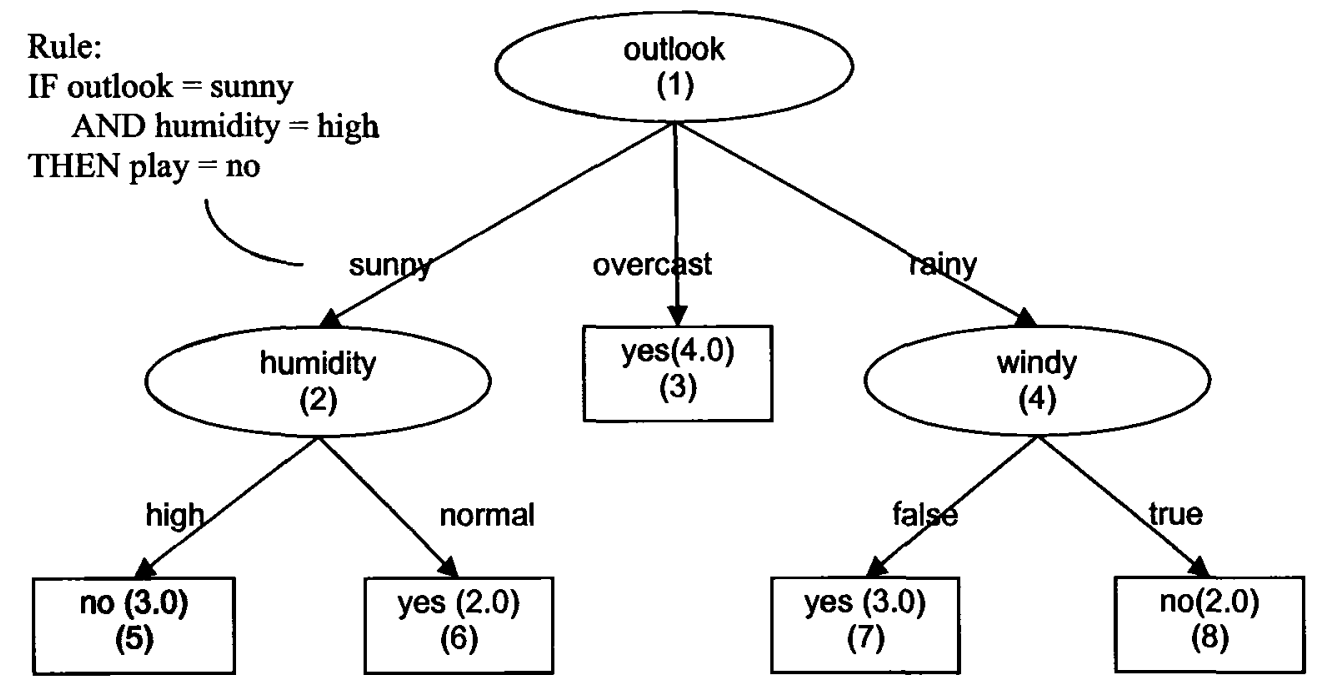

Figure 3-2 Decision tree for the weather data

\subsubsection{Model performance: Ten-fold cross-validation}

For classification problems, a classifier is the model, generated by a rule induction algorithm on a set of instances called training set, to classify the instances. The classifier predicts the class of each instance: if it is correct, that is counted as a success; if not, it is an error. It is natural to measure a classifier's performance in terms of the error rate [33], the proportion of errors made over a whole set of instances. Since the classifier is generated on the training set, it is common to apply the classifier on another set of instances named testing set and assess its error rate on this set. To make the evaluation objective, it is important that the training data was not used in any way to create the classifier [33].

When there are not enough instances for both training set and testing set, cross validation can be applied for rule induction [33]. The standard way of predicting the error rate of a learning technique is to use stratified ten-fold cross-validation [33]. The instances are divided randomly into 10 equal parts. Each part is held out in turn and the classifier is trained on the remaining nine-tenths; then its error rate is calculated on the holdout set. 
Thus the learning procedure is executed a total of 10 times on different training sets. Finally, the 10 error estimates are averaged to yield an overall error estimate.

There are also other ways of measuring the error rate of a learning schema [33]: leaveone-out cross-validation, bootstrap, etc. Since 10-fold cross-validation has become a standard method [33], in our experiments, we will use the ten-fold cross-validation to perform rule induction and evaluate the classifier's performance.

\subsubsection{Model performance: cost of error prediction}

Ten-fold cross validation evaluates the performance of a generated classification model. Besides the performance, the cost of making wrong classifications shall also be taken into account. Take the two-class prediction, which has two possible outputs (e.g. yes and no, pass and fail), as an example. A single prediction has four different possible outcomes:

true positive: the correct prediction of an instance when it is actually positive; true negative: the correct prediction of an instance when it is actually negative; false negative: an instance is incorrectly predicted as negative when it is actually positive;

false positive: an instance is incorrectly predicted as positive when it is actually negative;

To estimate the cost of error prediction, several popular criteria are used: true positive rate (TPR) and false positive rate (FPR) (section 3.2.3.1), ROC area (section 3.2.3.2), precision and recall (section 3.2.3.3), and F-measure (section 3.2.3.4). There are also other measures to be used to characterize performance of a model, such as the meansquared error, relative absolute error, correlation coefficient etc. For more details, please refer to Chapter 5 of [33]. 


\subsubsection{True positive rate and false positive rate}

$T P R$ and FPR are the simplest criteria for evaluating the cost of error-prediction. Let's suppose that $T P, T N, F P, F N$ denote the number of true positive prediction, true negative prediction, false positive prediction, false negative prediction respectively. Then the true positive rate (TPR) is TP divided by the total number of positives, and false positive rate (FPR) is FP divided by the total number of negatives. Please note that the summation of TPR and FPR is not $100 \%$.

$$
\begin{aligned}
& T P R=T P /(T P+F N) \\
& F P R=F P /(F P+T N)
\end{aligned}
$$

For cost estimation, each false prediction (false positive or false negative) will be assigned a cost value. The cost of incorrect predictions can be estimated by multiplying the $F P R$ (resp. $F N R$ ) with the $F P$ cost (resp. the $F N$ cost).

\subsubsection{ROC area}

ROC (Receiver Operating Characteristic) curves are a graphical technique for evaluating classifiers and depict the performance of a classifier without regard to class distribution or error rate [33]. They are usually used in a situation in which the learner is trying to select samples of instances that have a high proportion of positives (e.g. pass). One method to generate a ROC curve is to sort the instances in descending order of the predicted probability of a given class (e.g. pass). The number of positives (resp. negatives) included in the ordered set is plotted on the $\mathrm{Y}$-axis (resp. $\mathrm{X}$-axis), expressed as a percentage of the total number of positives (resp. negative).

Figure 3-3 is a sample of three ROC curves. A point $(x \%, y \%)$ in the ROC curve indicates 
that covering $\mathrm{y} \%$ of the true positives gives a false positive rate of $\mathrm{x} \%$. That is, if a learner want a $y \%$ of the true positives, he would possibly obtain $y^{*}{ }^{*} T P+x \% * F P$ instances. Usually, the accuracy of a test is evaluated by the area under the curve, or ROC area. Roughly, the larger the area is, the better the model is [33]. A rough guide for the accuracy of classification according to ROC area is the traditional academic point system $^{2}$ :

- $R O C$ area $\in[0.90,1]:$ excellent $(A)$

- $R O C$ area $\in[0.80,0.90): \operatorname{good}(B)$

- $\quad R O C$ area $\in[0.70,0.80)$ : fair (C)

- $R O C$ area $\in[0.60,0.70)$ : poor (D)

- $\quad R O C$ area $\in[0.50,0.60)$ : fail $(F)$

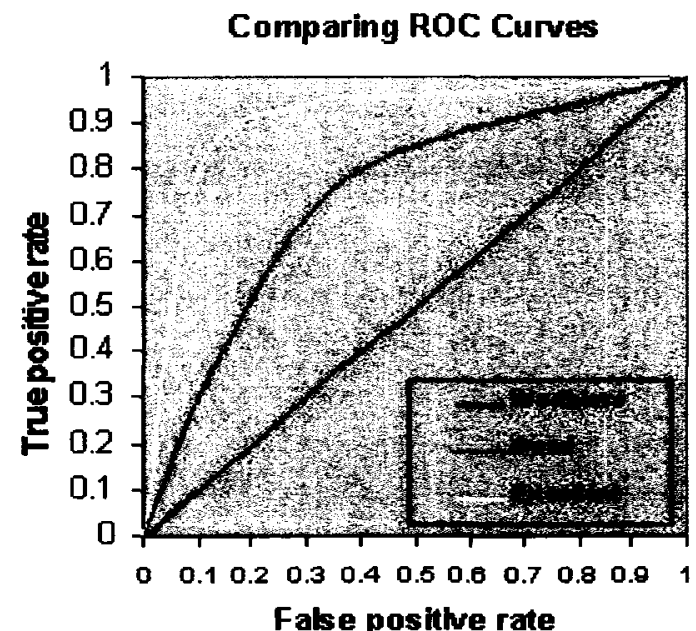

Figure 3-3 A sample ROC curve

\subsubsection{Precision and recall}

The precision and recall are applied to evaluate a model from the view of the relative cost of false positive and false negative. The precision is the percentage of true positives in the positive predictions; the recall is same as TPR. They are defined as:

$$
\text { precision }=T P /(T P+F P)
$$

\footnotetext{
2 http://gim.unmc.edu/dxtests/ROC1.htm
} 
recall $=T P R=T P /(T P+F N)$

\subsubsection{F-measure}

As precision and recall, $F$-measure is another measure used in information retrieval to characterize performance of a model:

$$
F-\text { measure }=\frac{2 \times \text { recall } \times \text { precision }}{\text { recall }+ \text { precision }}=\frac{2 \times T P}{2 \times T P+F P+F N}
$$

\subsection{Statistics background}

Mean value is one of the statistics used to evaluate the variance of a set of samples. Besides the mean values, the confidence intervals of the means are also used, because the estimates of the means vary from sample to sample. The interval estimates give an indication of how much uncertainty there is in our estimates of the true mean. The narrower the interval is, the more precise is our estimate ${ }^{3}$. A confidence interval generates a lower and upper estimated limit for the mean. Confidence limits are expressed in terms of confidence coefficients. Although the choice of confidence coefficient [29] is somewhat arbitrary, in practice $90 \%, 95 \%$, and $99 \%$ intervals are often used, with $95 \%$ being the most commonly used. In this thesis, we used the $95 \%$ confidence coefficient. The confidence intervals [29] are defined as:

$$
\bar{M} \pm t_{\alpha / 2, N-1} \frac{S}{\sqrt{N}} \text { and } S=\sqrt{\frac{1}{N-1} \sum_{l=1}^{N}\left(Y_{i}-\bar{Y}\right)^{2}}
$$

where $\bar{M}$ is the sample mean, $S$ is the sample standard deviation, $N$ is the sample size, $\alpha$ is the desired significance level (here, $\alpha$ equals to $5 \%$ ), and $t_{\alpha / 2, N-1}$ is the quantile of the $t$ distribution with $N-1$ degrees of freedom and can be obtained from the table of " $t$

\footnotetext{
${ }^{3} \mathrm{http}: / /$ www.itl.nist.gov/div898/handbook/eda/section3/eda352.htm
} 
Distribution with $n$ Degrees of Freedom" ${ }^{\text {"4. }}$. Note that the confidence coefficient is 1- $\alpha$.

${ }^{4}$ This table can be found in most of the statistical books. 


\section{CHAPTER 4 STATEMENT RANKING STRATEGIES}

In this chapter, we introduce several statement ranking strategies. We first provide some basic notation (Section 4.1). We then revisit statement coloring according to Tarantula's approach (Section 4.2). We then describe how we adjust Tarantula's ranking based on a C4.5 decision tree (Section 4.3), which is itself based on the notion of abstract test suite or test specification to account for the diversity of execution conditions under which statements are involved in failing test cases.

\subsection{Definition}

In order to facilitate the precise and concise definition of our methodology, we need to define and formalize a number of basic concepts.

The set of executable statements of the program $P$ being tested and corrected is referred to as $S . T$ is the test suite devised for testing program $P$ (set of test cases). $T_{F} \cup T_{P}=T$ where $T_{F}$ is the set of failed test cases $\left(T_{F} \subseteq T\right)$ and $T_{P}$ is the set of passed test cases $\left(T_{P} \subseteq T\right)$ : $T_{F} \cap T_{P}=\varnothing . S(t)$ denotes the statements executed (covered) by test case $t$, where $t \in T$ : $S(t) \subseteq S$

$R_{i}$ denotes a specific rule learned by the C4.5 algorithm. The set of rules is denoted by $R$, where $R_{i} \in R . R_{F}$ (resp. $R_{P}$ ) is the subset of rules that classifies test cases as Fail (resp. Pass): $R_{F} \cap R_{P}=\varnothing, R_{F} \cup R_{P}=R$.

The set of test cases classified by rule $R_{i}$ is referred to as $T\left(R_{i}\right): T\left(R_{i}\right) \subseteq T$. If $i<>j$, $T\left(R_{i}\right) \cap T\left(R_{i}\right)=\varnothing$. The latter constraint is due to the partitioning nature of the $C 4.5$ 
algorithm. Test cases in $T\left(R_{i}\right)$ can pass or fail. Following the same notation as above, $T\left(R_{i}\right)=T_{F}\left(R_{i}\right) \cup T_{P}\left(R_{i}\right)$ where $T_{F}\left(R_{i}\right)$ and $T_{P}\left(R_{i}\right)$ are the actual failing and passing test cases classified by rule $R_{i}$, respectively. The set of statements covered by a rule $R_{i}$, i.e., executed by the test cases classified by $R_{i}$, is referred to as $S\left(R_{i}\right): S\left(R_{i}\right)=\bigcup_{t \in T\left(R_{i}\right)} S(t)$.

\subsection{Tarantula coloring}

Following the above formalism, the Tarantula color of a statement $s$ of program $P$ is Color $(s)=p a s s e d(s) /($ passed $(s)+f a i l e d(s))$ where passed(s) and failed(s) are the percentages of passing and failing test cases that execute statement $s$ respectively $(\operatorname{Color}(s) \in[0,1])$ :

$$
\begin{aligned}
& \operatorname{passed}(s)=\left|T_{P}(s)\right| /\left|T_{P}\right|=\left|\left\{t \in T_{P}, s \in S(t)\right\}\right| /\left|T_{P}\right| \\
& \operatorname{failed}(s)=\left|T_{F}(s)\right| /\left|T_{F}\right|=\left|\left\{t \in T_{F}, s \in S(t)\right\}\right| /\left|T_{F}\right|
\end{aligned}
$$

where $T_{P}(s)$ and $T_{F}(s)$ are the set of passing and failing test cases that execute statement s respectively.

A small value for Color(s) suggests $s$ is a suspicious statement. When statements are not executed by any test case, the authors $[14,15]$ states that a value of zero should be assigned to Color(s). (Note that this is what the description of Tarantula suggests in [14, 15], although the running example used by the authors suggests that, on the contrary, uncovered statements are not ranked.) However, this makes no theoretical sense as there is no information to indicate that the unexecuted statement is likely to be fault-prone. In fact, no fault causing the observed failures can be possibly located in uncovered statements. This situation is of practical importance as the presence of multiple faults often prevents certain statements from being executed. In our case study, we will discuss 
a stepwise debugging process to address this issue of uncovered statements (Chapter 5).

The original Color(s) formula presented above can be re-expressed to demonstrate that the statement ranking only depends on the ratio $\left|T_{P}(s)\right||| T_{F}(s) \mid$.

$$
\frac{1}{\operatorname{Color}(s)}=1+\frac{\left|T_{F}(s)\right|}{\left|T_{F}\right|} * \frac{\left|T_{P}\right|}{\left|T_{P}(s)\right|}=1+\frac{\left|T_{P}\right|}{\left|T_{F}\right|} * \frac{\left|T_{F}(s)\right|}{\left|T_{P}(s)\right|}
$$

Since $\left|T_{P}\right| / T_{F} \mid$ is constant for a given test set and program version, the ranking of statements, which is ultimately used to focus debugging, exclusively depends on the proportion of test cases that fail and pass when executing a specific statement $s$. Though this makes intuitive sense, there are two problems with this formula in a multi-fault environment:

(1) Test cases may fail due to different faults and therefore a statement $s$ may not be relevant to the failing test cases that execute it.

(2) Redundant test cases that execute the system in identical or similar conditions may artificially affect the ranking.

Another issue is related to how Tarantula rankings were evaluated (section 2.1). Ideally, we would like to assess the cost-effectiveness of rankings. In other words, we want to be able to compare some measure of cost and fault detection. To do so we use a scatter-plot of percentages of statements verified (a surrogate measure for cost) versus percentages of faults contained in those statements (effectiveness) to compare various statement-ranking methods in terms of cost-effectiveness. Though this was not done in the original studies $[14,15]$, this will be the basis for our case study analysis.

Also related to evaluation is whether each fault should be considered in isolation or whether all faults should be considered in a single program. Tarantula has originally been 
used on faulty program versions with a single fault $[14,15]$ and found to be effective at pinpointing faulty statements on such faulty programs. When used on multiple-fault programs, Tarantula's effectiveness has shown to decrease [15], probably for the reasons we mentioned above. As acknowledged in [15], this deserves further analysis and is important as it is probably more realistic to perform such studies with multiple faults. This issue is the main focus of the present thesis.

\subsection{Ranking statements based on $\mathrm{C} 4.5$ rules}

In this section we present the rule-based statement ranking (RUBAR) method. We first explain how we apply the Category-Partition technique to generate C4.5 rules (section 4.3.1) and how we select a subset of rules based on all the rules available in the decision tree (section 4.3.2). We then explain the basic principles of using $\mathrm{C} 4.5$ rules for deriving a ranking (section 4.3.3). Finally, we introduce the debugging process to apply the RUBAR fault localization technique (section 4.3.4).

\subsubsection{Generating a $\mathbf{C} 4.5$ classification tree}

In order to obtain meaningful and accurate machine learning rules, we cannot simply use the raw input and output values of a test case. Our experience is that they typically lead to meaningless and inaccurate rules because the machine learning algorithm cannot learn what input or output properties are potentially of interest but only which ones matter once they are defined. In other words, without some additional guidance, the learning algorithm is unlikely to find the precise conditions under which test cases fail. Figure 4-1 is a sample of decision tree generated from the raw test data (original test case) in Table A-1 for the Triangle program (section 3.1, Appendix A). It shows that if side $1\left(s_{1}\right)$ is 
smaller or equal to 2 and side $3\left(s_{3}\right)$ is smaller or equal to 5 , which corresponds to four test cases (leaf node of the left rule), then the program fails, i.e., misclassifies the triangle. Similarly (right rule), if side $1\left(s_{l}\right)$ is strictly greater than 2 , the program fails. It is obvious that the information provided by the classification tree it too close to the raw value. That is, the information is too subjective instead of objective. For example, instead of the second rule discussed earlier (Figure 4-1), we would like to say that when a triangle has one side longer than two (not only side $s_{1}$ ), the triangle program may misjudge the type of the triangle.

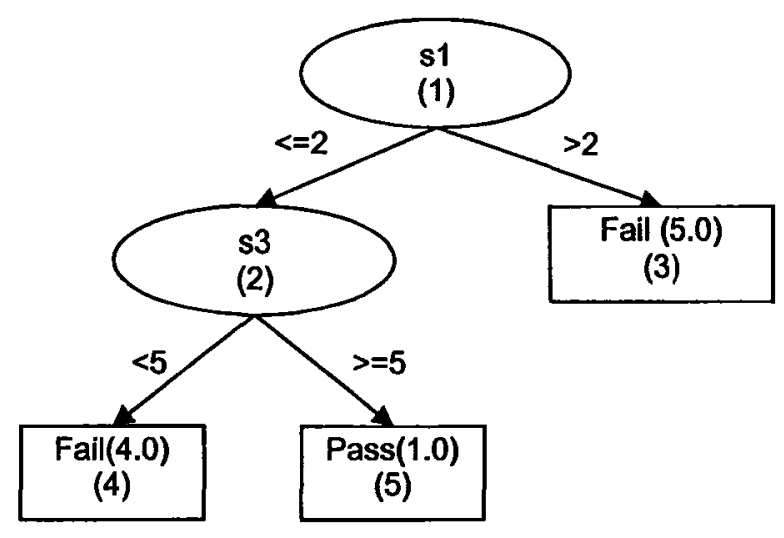

Figure 4-1 Decision tree generated on raw inputs

Therefore, if no black box testing technique is used beforehand to obtain a test specification, test suites (raw test data) need to be transformed into abstract test suites before rule-induction machine learning algorithms can be applied. The abstract test suite contains the abstract test cases which define the test specifications of the original test cases (raw test data). In our method, we apply the Category-Partition (CP) method to define the test specifications (by categories and choices) and then use the categories and choices to transfer the original test suite into an abstract one.

The reason to use Category-Partition (CP) here is that $\mathrm{CP}$ is more general and 
encompassing than equivalence class partitioning (which only partitions input value domains) and it is one of the most well-known black-box techniques. Though applying $\mathrm{CP}$ clearly represents an overhead, in many environments one would be expected to use a systematic functional testing approach. Reverse engineering a test specification (i.e., categories and choices) is also in any case a useful investment in the context of legacy systems with existing test suites.

Let's take the triangle problem (Appendix A) as an example. Using the category partition definitions of Table 3-1, the original test cases in Table A-1 can be defined by the abstract data in Table A-2. Using the abstract test cases, we can generate a decision tree as Figure 4-2. Figure 4-3 is the graph view of Figure 4-2. From the rules of this tree, we can clearly draw out that when the sides $\left(s_{1}, s_{2}, s_{3}\right)$ of a triangle have relationship $s_{1 \leq}\left|s_{2}-s_{3}\right|$, the triangle program would misclassify the triangle. This is exactly what the fault is.

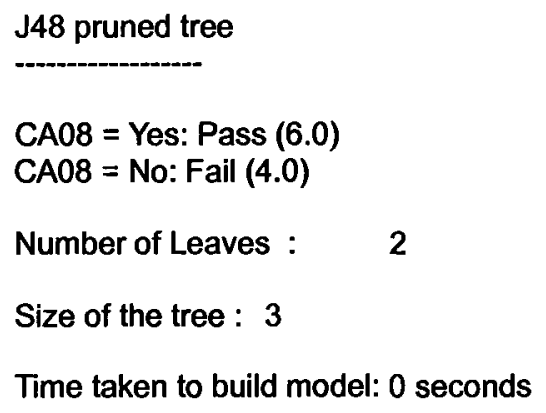

Figure 4-2 Decision tree of Triangle program generated by Weka

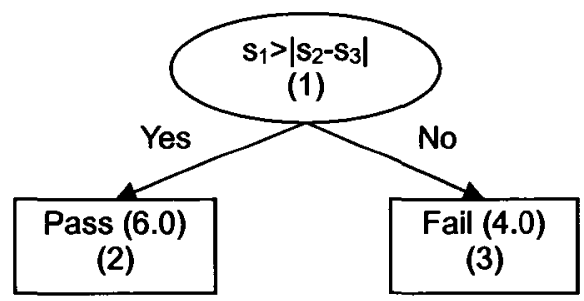

Figure 4-3 Decision tree generated on complete CP definition 
When applying the CP methodology, one must provide a complete CP definition for the program. The completeness of the $\mathrm{CP}$ definition will affect the accuracy of the rules learned directly. Still taking the Triangle program as the sample, suppose that instead of taking all the CP definitions in Table 3-1, we only take the first three categories (CA01, $\mathrm{CA} 02$ and $\mathrm{CA} 03$ ) and related choices as the $\mathrm{CP}$ definitions of the triangle program. Based on the new abstract test cases and test results, the learning algorithm will be able to generate a C4.5 tree in Figure 4-4, which determines that a test case with inputs $s_{1}<s_{2}$ and $s_{3}>s_{1}$ has a $66.7 \%(2 / 3)$ probability to detect a failure (node 5). It is obvious that the decision tree of Figure 4-3 is more accurate and meaningful than that of Figure 4-4. That is, the completeness of the CP definition is one of the key features in generating an accurate classifier.

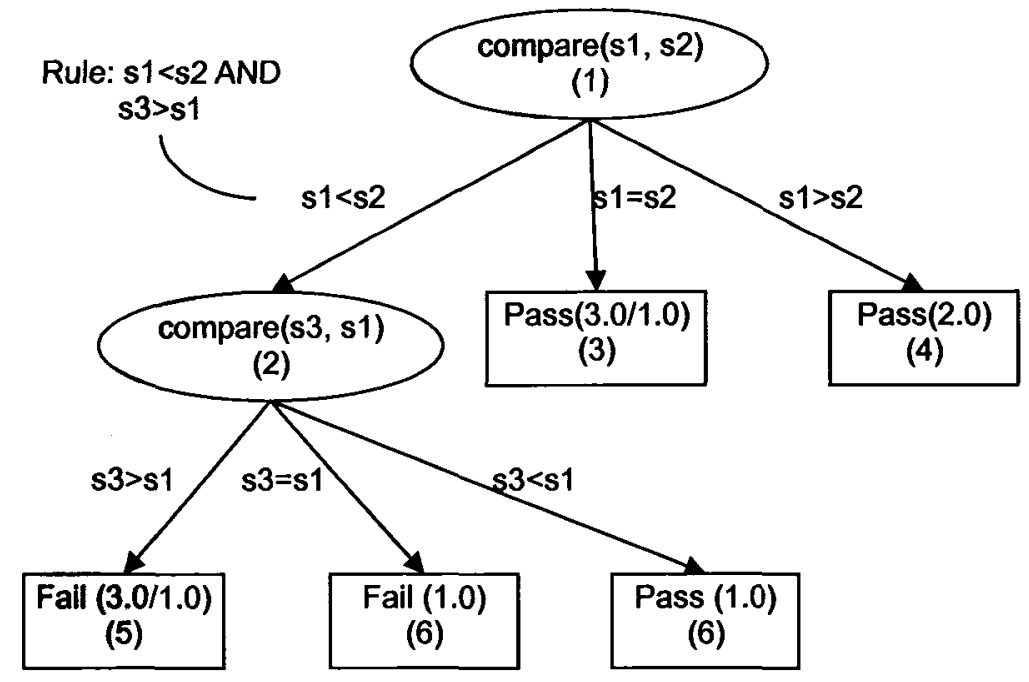

Figure 4-4 Decision tree generated on non-complete CP definition

When applying the J48 classification algorithm of Weka, the parameters of the algorithm should be chosen carefully. Our experience shows that if there is only a small set of instances, it is better to run the J48 algorithm with the default values for the parameters: 
the confidenceFactor (the confidence threshold for pruning) equals to 0.25 , the minNumobj (the minimum number of instances permissible at a leaf) equals to 2 , the numFolds (the size of the pruning set) equals to 3 , etc. But when there is a large set of instances (such as the SPACE program introduced in section 5.2), our experiences reveal that when the minNumobj equals to 10 , and the other parameters equals to their default values, Weka can generate a more accurate classification tree.

\subsubsection{Selecting rules}

A last practical issue is to determine which rules to consider among all the ones identified in a C4.5 decision tree. In order to only account for accurate rules, it seems logical to select rules that are above a certain probability of correct classification (Fail/Pass). We should also consider rules that are based on a large enough number of instances (abstract test cases) in order to avoid the classifications that are due to chance. Deciding about such selection thresholds is of course subjective, but it can be done so as to obtain a reasonable number of rules to base the ranking on and will be dependent on how accurate the rules are overall for the specific test suite under consideration.

\subsubsection{Computing a statement ranking}

\subsubsection{Heuristics}

Assuming we have an abstract test suite, we are able to generate a $\mathrm{C} 4.5$ decision tree where each leaf corresponds to a rule predicting either Pass or Fail. The data set on which to build the tree would then be a set of instances, each corresponding to an abstract test case that is known to pass or fail. The attributes to be selected for the tree construction are pairs (category, choice) characterizing the specification of each test case. Each path 
from the root of the tree to a leaf represents a rule characterized by conditions on (category, choice) pairs. Each leaf in the tree corresponds to a partition of the data set of test case instances as introduced in section 4.3.1. A rule leads to a Fail prediction when the instances in its leaf represent a majority of fail test cases, and Pass otherwise. Therefore, a C4.5 rule classifies a set of test cases with similar input conditions and identical test results.

To understand the heuristic we are going to follow, let us first focus on Fail rules and take the example of a particular rule $R_{i} \in R_{F}$. If we assume the probability of failure associated with $R_{i}$ is very high, then what this means is that (nearly) all the test cases in $T\left(R_{i}\right)$ fail under the same or similar conditions. What this suggests is that (nearly) all of these test cases fail due to similar reasons (faults). This in turn can be used to safely rank statements using a strategy similar to Tarantula as all test cases failing within a rule can be safely assumed, in most cases, to fail due to the same fault(s). We thus obtain a ranking for each Fail rule that must then somehow be combined with the ranking of other rules to obtain a final ranking.

Within a failing rule (e.g. $R_{i}$ ), the higher the number of failing test cases covering a statement, the more suspicious the statement. For instance, assume that $T_{F}\left(R_{i}\right)=\left\{t_{1}, t_{2}, t_{3}\right\}$, and that the statements executed by these three test cases are depicted in Figure 4-5. $D_{1}$ is the set of statements executed by all three test cases; $D_{2}$ is the set of statements executed by two test cases; $D_{3}$ is the set of statements executed by only one test case and $D_{4}$ is the set of statements executed by none of the test case of rule $R_{i}$. According to the above hypothesis, the statements in $D_{1}$ (i.e., covered by all three test cases) should be more suspicious than those in $D_{2}, D_{3}$, or $D_{4}$. On the contrary, the statements in $D_{3}$ should be the 
safest of all statements covered by $t_{1}, t_{2}, t_{3}$. Since no failed test case executes the statements in $D_{4}$, it is hard to point out the degree of suspicion of these statements. Similarly, if $R_{i}$ were a Pass rule, we would obtain the opposite result: the statements in $D_{l}$ would be the safest and those in $D_{3}$ would be the most suspicious. This example illustrates how for each rule we can obtain a partial ordering of statements.

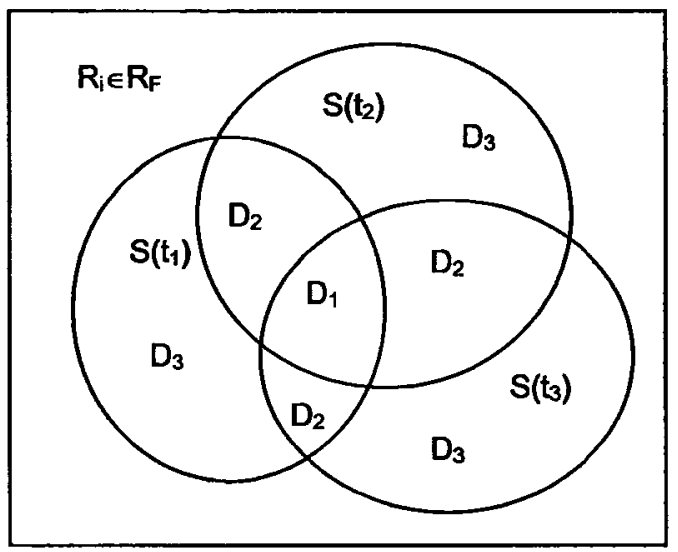

\section{Figure 4-5 Statement divisions by test cases}

\subsubsection{Ranking statements}

To implement the above heuristic, we must define a mechanism to combine the ranking of all rules for a given statement. Proceeding with this goal in mind, a statement will be assigned a negative (resp. positive) weight if it is covered by a Fail (resp. Pass) rule. A high absolute weight value implies that a statement is executed by most of the test cases in the rule. The absolute values of weights are normalized within $[0,1]$ in order to give equivalent weight to all perfect rules, i.e., rules which contain only test cases that either fail or pass. However, if a rule contains test cases with inconsistent Pass/Fail behavior, the maximum absolute weight a rule can contribute will therefore be below 1 and determined by the percentage of test cases it contains in the Fail/Pass category it predicts. The fact that rules should contribute to the extent of the consistency of their test cases' 
behavior should be intuitive: less consistent rules have less influence on the final ranking. We have also considered weighting the rules' contributions according to their number of test cases, but this would make the results sensitive to redundant test cases, as discussed above in the case of Tarantula, and would make rarely executed faults difficult to find. Assuming that Weight $\left(R_{i,}, s\right)$ and Weight(s) denote the weight of statement s for rule $R_{i}$ and the overall weight of statement $s$, respectively, we obtain:

$$
\begin{aligned}
& \text { Weight }\left(R_{i}, s\right)=-\left|T(s) \cap T_{F}\left(R_{i}\right)\right| \backslash T\left(R_{i}\right) \mid, \text { when } R_{i} \in R_{F} \\
& W \text { eight }\left(R_{i s} s\right)=\left|T(s) \cap T_{P}\left(R_{i}\right)\right| / T\left(R_{i}\right) \mid, \text { when } R_{i} \in R_{P} \\
& \text { Weight }(s)=\sum_{R_{i} \in R} \text { Weight }\left(R_{i}, s\right)
\end{aligned}
$$

This can be visualized as a matrix (Table 4-1) where columns are statements and rows are rules. The matrix contains all the weights of each statement for each rule, all within $[-1,1]$ as defined above. The last row represents the sum of all rule weights for a given statement, which can then be used to rank statements as depicted in Figure 4-6: Statements with lower weight are ranked first, to follow Tarantula's convention for Color(s).

Weight(s)

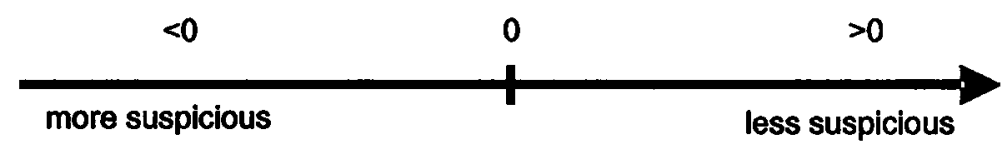

Figure 4-6 Statement Ranking using Weight(s) 
Table 4-1 Matrix of statement weight

\begin{tabular}{c|ccccc}
\multicolumn{1}{c}{} & $\mathrm{s}_{1}$ & $\mathrm{~s}_{2}$ & $\mathrm{~s}_{3}$ & $\ldots$ & $\mathrm{s}_{0}$ \\
\cline { 2 - 6 } $\mathbf{R}_{\mathrm{P} 1}$ & 1 & 0.01 & 1 & $\ldots$ & 0.5 \\
$\ldots$ & $\ldots$ & $\ldots$ & $\ldots$ & $\ldots$ & \\
$\mathbf{R}_{\mathrm{Pm}}$ & 1 & 1 & 0.8 & $\ldots$ & 0.1 \\
$\mathbf{R}_{\mathrm{F} 1}$ & -1 & -1 & -1 & $\ldots$ & -1 \\
$\ldots$ & $\ldots$ & $\ldots$ & $\ldots$ & $\ldots$ & $\ldots$ \\
$\mathbf{R}_{\mathrm{Fn}}$ & -1 & -1 & -0.8 & $\ldots$ & 0 \\
\hline Sum & -22.1 & -18.7 & 0.06 & $\ldots$ & -3.36
\end{tabular}

\subsubsection{Iterative debugging process}

The debugging process of a program with several faults is more complex than that of a program with one fault. The presence of multiple faults often prevents certain statements (possibly faulty themselves) from being executed. As a result, not all faults can be covered in a single run. On the other hand, it is also impossible for a programmer to fix all the faults in the executed statements of a single rule. Therefore, the debugging process of a multi-fault program must follow an iterative process, which can be described by Figure 4-7. Suppose that a set of test cases is defined to meet the test requirement and all faults in the program can be recovered by at least one of those test cases. Diagnosing a multi-fault program using the RUBAR technique entails to:

(1) Define the category partition definitions of the program and apply the "test case transformation tool" to generate an abstract test suite;

(2) Test cases are executed against the system-under-test, which may contain several faults. The test results (Passing or Failing) and execution traces are collected;

(3) A rule generation tool, i.e. Weka, is applied to generate rules with the abstract test cases and the related test results; 
(4) The RUBAR statement-ranking algorithm is applied to generate a ranking of the statements based on the coverage data and the classification rules;

(5) Following a fault removing strategy, one or more faults in the executed statements are removed and a new program version containing the left-over faults becomes available for another debugging process (steps 2 to 5 are repeated).

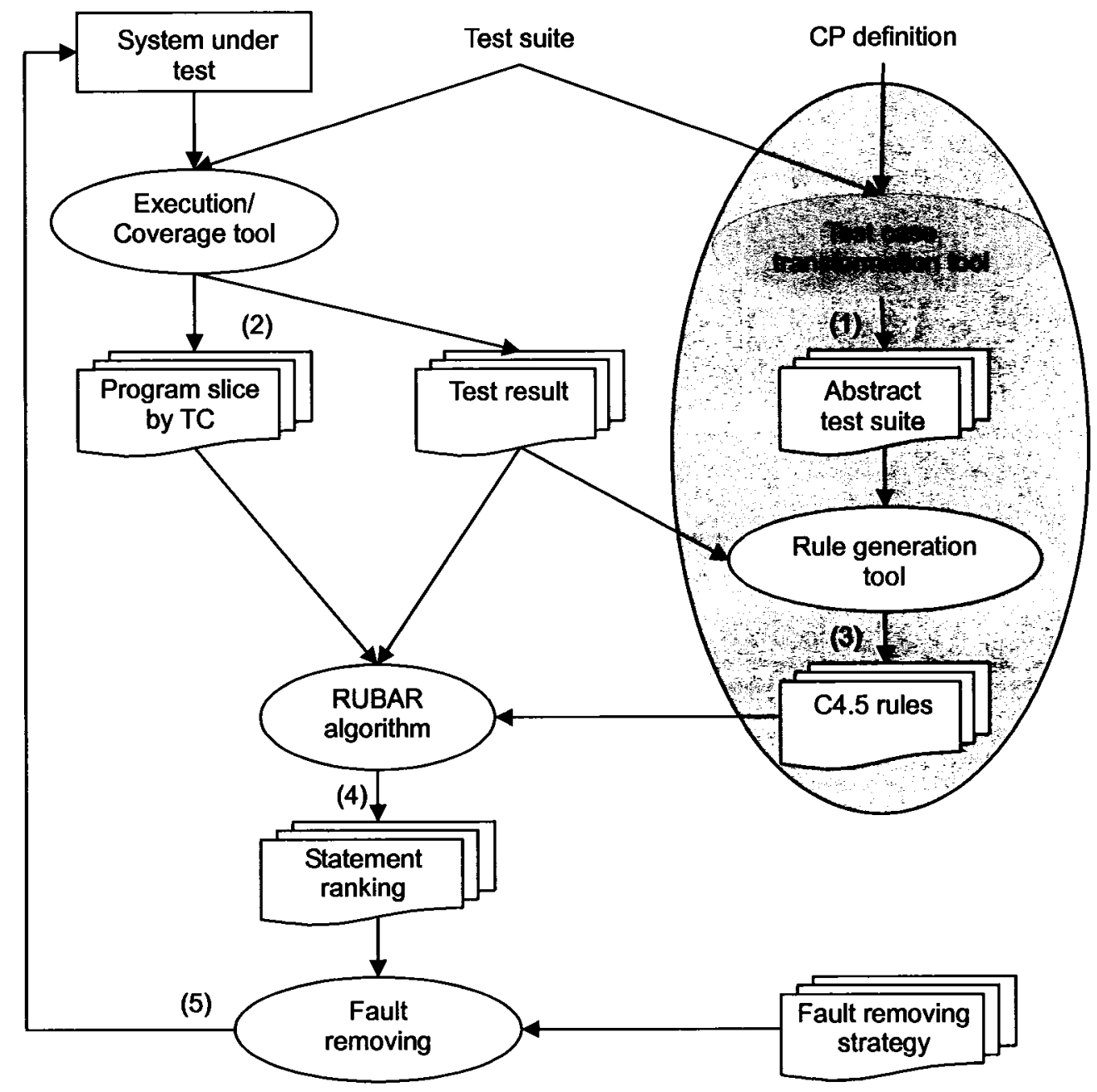

Figure 4-7 Iterative debugging process

The iterative debugging process (Figure 4-7) can be automated by using specific tools. There are lots of commercial and open-source coverage tools that we can rely on to 
record the execution trace of each test case, e.g. Emma ${ }^{5}$ and Clover ${ }^{6}$ for Java, Coverage Validator $^{7}$, Telcordia XSuds ${ }^{8}$ and IBM-Rational PureCoverage (now the Rational purifyPlus) for $\mathrm{C} / \mathrm{C}++$. In our experiments, we used Emma and Coverage Validator as the coverage tools for Java language and C language separately. Weka [33] is an open-source Java-based machine learning package for data mining tasks. Weka contains tools for data pre-processing, classification, regression, clustering, association rules, and visualization. The Tarantula algorithm and RUBAR algorithm were also implemented in Java language (Appendix B is the class diagram of the implementation).

To correct the errors in the executed statements (Step 5) before iterating the process, several strategies can be followed:

(1) All the ranked statements are analyzed and any fault discovered fixed;

(2) Only the first $20 \%$ of the ranked statements are analyzed and any fault discovered fixed. Investigating the first $20 \%$ of the ranked statements has been shown to be a reasonable heuristic with Tarantula [15];

(3) All the ranked statements are analyzed and only the first fault discovered is fixed;

(4) Any other procedure deemed suitable by the tester can be followed.

It is up to the tester to decide which one of the above procedures to follow.

\subsection{Manual Effort and Automation}

From the discussion above, the category partition definitions should be as complete as

\footnotetext{
${ }^{5} \mathrm{http}: / /$ sourceforge.net/projects/emma

${ }^{6} \mathrm{http}: / / \mathrm{www}$.atlassian.com/software/clover/

${ }^{7} \mathrm{http}: / /$ www.softwareverify.com/cpp/coverage/index.html

${ }^{8} \mathrm{http}: / / \mathrm{xsuds}$.argreenhouse.com/
} 
possible. This requires a thorough understanding of the system domain. This is what a tester would typically do when trying to locate faults.

Once the CP specification is defined, the transformation of raw test cases into abstract test cases is easy to be automated. Usually, it takes only a couple of seconds to perform the transformation. For instance, for a large program such as the Space program (section 5.2), for which we defined 83 categories and 582 choices, it took less than a minute to transform the 13,585 test cases into abstract test cases.

The second debugging step, executing test cases and collecting coverage data, may be the most time-consuming activity of the iterative debugging process described by Figure 4-7. The time to collect the coverage data and test results may range from several hours to several days, which depends on the size of the program and the size of the test suite. In practice, most companies use code coverage as one of the criteria to evaluate test performance. Therefore, coverage information becomes a by-product of the testing process. Therefore, the cost of collecting coverage data is not really part of the cost of our strategy.

Debugging step 3 is another automated step. We used the WEKA tool, which implements the C4.5 classification algorithm. For the Space problem, it took eight seconds to generate a tree based on the 13,585 abstract test cases.

As mentioned in section 4.3.4, we developed the RUBAR ranking algorithm, which takes the test results, the coverage data and the rules generated as the inputs. The ranking process can also proceed very quickly. It is obvious that step 5 is a manual process, as this relies on the knowledge and the experience of the programmer. However, as discussed in this chapter, the statement ranking can provide guidance for defect removal. 


\section{CHAPTER 5 CASE STUDIES}

Our experiments include two case studies which aim at validating RUBAR (section 4.3) and at comparing its effectiveness with Tarantula. This chapter starts with a presentation of the rule-based diagnosis process we used (section 5.1). Then we represent the two case study programs we used and the corresponding results (section 5.2 and section 5.3).

\subsection{Case study process}

As mentioned above, we apply the Weka tool to generate the $\mathrm{C} 4.5$ classification trees and then generate the rules indicating failing or passing conditions of a test case. To select the rules generated by the C4.5 algorithm, in our case study, we select rules with a probability of correct classification above 0.8 and a number of instances above 10 .

The case studies followed the iterative process suggested in section 4.3.4. To better evaluate the effectiveness of RUBAR algorithm, we performed the first case study (the Space program) on two scenarios defined in section 4.3.4:

(1) Inspecting all the ranked statements and fixing any fault discovered;

(2) Inspecting the first $20 \%$ of the ranked statements and fixing any fault discovered;

Since not all statements can be executed in a multi-fault environment and the purpose of software debugging was to identify the location of faults generating the observed failures, we only ranked the unfixed statements since other statements could not possibly contain the faults causing these failures.

After all the faults are located and removed, we appended the rankings of all debugging 
iterations and compared the resulting final rankings of all program statements as determined by Tarantula and RUBAR. Note that for each debugging iteration, we only ranked the statements not fixed in the former iteration(s). We then generated a diagram with the statement ranking against the cumulative percentage of faulty statements in the ranking to evaluate the effectiveness of the ranking. We refer to this diagram as the costeffectiveness diagram.

As discussed above, Baudry et al. [3] presented the idea of using the size of DDB (Dynamic basic block) to evaluate the diagnosis accuracy. They validated the relationship by their case study and defined a criterion to measure the accuracy of diagnosis (section 2.2.3). In our case studies, we applied these criteria together with the cost-effectiveness diagram to evaluate the accuracy of the Tarantula and RUBAR methods.

Another important aspect of the case studies is whether the decision tree and rules we generated are accurate. This is of course a prerequisite for such rules to be used for statement ranking purposes but also to assess whether we can characterize failure conditions (Fail rules) in an accurate manner. This will be further discussed below.

\subsection{Case study 1: The Space program}

\subsubsection{Introduction}

The Space program was originally developed by the European Space Agency, and was first used in a software engineering study by Pasquini et al. [23]. It has subsequently been used in other experiments, and in particular in the initial evaluation of Tarantula [15]. Space allows users to describe the configuration of an array of antennas using a specific array definition language (ADL). It reads a text file containing ADL statements, checks 
its conformance to the ADL grammar as well as specific consistency rules, and performs other computations. It is a 5905-NBNC ${ }^{9}$ - LOC C program. (See [34] for further details.) During "testing and operational use" of the program, 33 logical faults were identified and eliminated, and the details of the fault-fixing changes were preserved so that the faults could be selectively re-introduced. Vokolos and Frank1 [30] used the program for a study in which they compared the effectiveness of regression test selection strategies. For that study, they generated 10,000 test cases using a randomized input generation tool. Rothermel et al. [28] later added enough test cases to ensure that each executable decision was covered by at least 30 test cases in each direction; this procedure added 3,585 test cases to the pool. The resulting test pool covers $90,85,85$, and 80 percent of all blocks, decisions, c-uses, and p-uses present in the program, respectively. The total number of blocks, decisions, c-uses, and p-uses are 2995, 1191, 3733, and 1707, respectively.

During the course of their research, Rothermel et al. [28] identified and eliminated five more faults, bringing the total number of versions of the program to 38 ; however, they found that three of the original versions did not exhibit faulty behavior, reducing the number of non-equivalent versions to 35 . We obtained the program, faulty versions and test pool from the Galileo Research Group Subject Infrastructure Repository at the University of Nebraska - Lincoln. In this case study, we used the Space program [23], the mutants and test cases generated by the authors of $[23,28]$ directly.

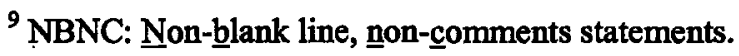




\subsubsection{A multi-fault version of Space}

Among the 35 non-equivalent versions, there are two special versions that contain faults in the initialization statements. Initialization statements are statements to initialize the global variables or to set up the system environments, which will be executed by almost all the test cases. In [15], Jones point out that Tarantula can not correctly color (i.e., rank) those statements. Baudry et al. [3] confirmed this situation in their case study. Our case studies also reveal that RUBAR, similarly to Tarantula, does not work well in locating the defects in the initialization statements. Therefore, in the following case studies, we chose not to introduce faults in the initialization statements as Baudry et al. did in their case study.

We randomly selected 15 of the 33 original logical faults for our study, avoiding the versions not exhibiting any faulty behavior and containing the initialization fault. We then built one faulty version of Space by introducing, in the correct version, the 15 logical faults, i.e., 34 faulty statements. The rationale was to simulate a realistic situation where a program contains more than one fault. At the same time, the rationale for not including all the 33 original faults was to obtain a balanced number of failing and passing test cases. The failing rate of test cases on a faulty version including the 33 faults is $95.5 \%$, whereas the rate is $47 \%$ on the faulty program containing the 15 selected faults. Future work will investigate ways to deal with high failing rates and initialization faults.

When executing the 13,585 test cases on our multi-fault version of Space, we achieve $69.72 \%$ statement coverage (as reported by Coverage Validator). The executed statements contained 15 of the 33 faulty statements. Not reaching $100 \%$ statement coverage and not executing the 33 faulty statements is not surprising since we have several faults in the 
program. Some faults result in the program exiting prematurely, and therefore in some of the statements (containing other faults) not being executed. This, however, can be considered a realistic situation under which to experiment.

\subsubsection{Rule induction based on category partition}

In our method, we used the category partition technique to characterize the test cases instead of using this technique to devise test cases. Therefore, it is not necessary to identify constraints on choices as suggested by the category-partition technique [21]. When applying category-partition on the Space program, we identified 207 parameters, 83 categories, and 582 choices (Appendix C.2). Since the Space program is a language processing program, it is not surprising that we defined such a large number of categories and choices.

By applying the $\mathrm{C} 4.5$ algorithm on the test suite (with test results against the initial version of Space containing all 15 faults), we obtained 285 rules (decision tree leaves). The maximum depth of the tree is 12 . That is, the longest rules generated have 12 decisions (conditions). Overall, when performing the ten-fold-cross-validation procedure [33], we obtain the confusion matrix in Figure 5-1. From the matrix, we learn that the C4.5 algorithm misclassified 335 Fail test cases and 550 Pass test cases as Pass test cases (false positive) and Fail test cases (false negative) respectively. For Fail test cases this corresponds to a $91.7 \%$ precision and a $94.7 \%$ recall, which is very good. Similar percentages are obtained for Pass test cases. What this means is that the rules learned by the decision tree model in a nearly complete and precise manner the conditions under which the Space program fails. This result partly reflects the completeness of our categories and choices. 


\begin{tabular}{llrr} 
& & \multicolumn{2}{c}{ Predicted class } \\
& & Fail & Pass \\
Actual & Fail & 6045 & 335 \\
class & Pass & 550 & 6655
\end{tabular}

Figure 5-1 Cross validation confusion matrix

The accuracy does of course vary across rules as well as the number of test cases they cover. Table 5-1 shows examples of a few Fail rules with their length (\# of conditions), the number of test cases they covered (TC), and the accuracy of prediction. The rules we show in Table 5-1 are 17 of the 34 Fail rules that involve more than 10 test cases and achieve an accuracy of at least $80 \%$. Remember that we need enough instances associated with rules to obtain accurate fail/pass probability estimates. According to the rule selection criteria, we also selected 58 Pass rules from the rules generated by the $\mathrm{C} 4.5$ algorithm.

Table 5-1 Example Fail rules with accuracy and test case coverage

\begin{tabular}{|l|r|r|r|l|r|r|r|}
\hline Rules & length & TC & Accuracy & Rules & length & TC & Accuracy \\
\hline F109 & 1 & 30 & $100.00 \%$ & F004 & 11 & 200 & $94.50 \%$ \\
\hline F025 & 5 & 667 & $99.55 \%$ & F012 & 11 & 97 & $93.81 \%$ \\
\hline F104 & 2 & 240 & $98.75 \%$ & F005 & 11 & 548 & $93.80 \%$ \\
\hline F106 & 1 & 304 & $98.36 \%$ & F070 & 10 & 271 & $93.73 \%$ \\
\hline F105 & 2 & 158 & $97.47 \%$ & F069 & 9 & 116 & $92.24 \%$ \\
\hline F107 & 1 & 114 & $97.37 \%$ & F087 & 9 & 199 & $91.96 \%$ \\
\hline F009 & 12 & 147 & $97.28 \%$ & F016 & 7 & 12 & $91.67 \%$ \\
\hline F010 & 12 & 132 & $96.97 \%$ & F086 & 8 & 700 & $91.57 \%$ \\
\hline F003 & 12 & 37 & $94.59 \%$ & \multicolumn{5}{|c}{} \\
\cline { 1 - 5 } & & &
\end{tabular}

As an example, let us look at the rule F025 (Table 5-1). It has a length of five, i.e., it is made of five conditions. 667 test cases are classified by this rule. The failing probability of a test case satisfying the conditions is $99.55 \%$. Without going into too many details about the specification of the Space program, or a detailed description of our parameters, categories, and choices (i.e., our application of category-partition), rule F025 indicates that if a test case: 
1) defines a triangular grid of antennas (condition 1),

2) defines a uniform amplitude and phase of the antennas (conditions 2 and 3),

3) defines the triangular grid with angle coordinates or Cartesian coordinates, and a value is missing when providing the coordinates (conditions 4 and 5);

then, the test case has a failure probability of $99.55 \%$.

As shown in Table 5-1, many other rules are highly accurate in predicting failure. Note that such information can potentially be useful to support debugging (i.e. simplified inputs) as it characterizes logically and precisely failure conditions. Since the confusion matrix in Figure 5-1 suggests the rules are very accurate, they can therefore be trusted by a programmer trying to understand when and why the program fails.

From the abstract test suite generated, we identified that:

(1) There are a number of duplicated test cases. Remember that Rothermel et al. [28] generated some test cases to make each executable decision covered by at least 30 test cases in each direction. From the discussion above, these duplicated test cases will dramatically affect the effectiveness of the Tarantula fault localization method. We will use a test suite with less redundancy in the second case study.

(2) These 13585 test cases do not cover all the functions of the Space program, although they meet the coverage requirement [28] of Rothermel et al.

\subsubsection{Cost-Effectiveness analysis}

In the first iteration of the RUBAR procedure only $69 \%$, as discussed above, of the statements were reachable. As mentioned in section 5.1, we correct the statements ranked in two strategies: all the faults in the executed statements were found and corrected or only the faults in the first $20 \%$ most fault-prone statements were inspected and corrected. 
After that, in the subsequent debugging iterations, we only focus on the remaining statements that were left out in the former iteration(s). Finally, the statement rankings of all iterations are appended together.

\subsubsection{Strategy one: fix all faults in the ranked set of statements}

By this strategy, it took two debugging iterations to cover all statements, following the iterative process described in Section 5.1. The cost-effectiveness of the ranking of statements based on Tarantula's coloring is shown in Figure 5-2 (a) when combining the rankings of the two debugging processes (separated by the reference line of the $\mathrm{X}$-axis). The separated ranking of each iteration is showed in Appendix C.3.1. The first iteration involves all the 15 faults (34 faulty statements) whereas the second iteration includes only eight faults (18 faulty statements) that are not ranked in the first iteration. Table 5-2 contains the detailed data about the debugging iterations. The $\mathrm{X}$-axis is the percentage of statements that need to be covered to detect the percentage of faulty statements on the $\mathrm{Y}$ axis. Note that the rankings stop before $100 \%$ statement coverage as this is also the case for the original test suite.) Each observation represents a statement which is assigned a color value and the statements are ranked according to the color value. For example (Figure 5-2 (a)), by visiting the first $10 \%$ (resp. 20\%) of the covered statements, a tester will visit $17 \%$ (resp. 17\%) of the faulty statements. Figure 5-2 (b) shows the same curve when using RUBAR for ranking statements: the pattern is visually very different from Figure 5-2 (a), especially in the first iteration. 
Table 5-2 Execution result of multi-fault version

\begin{tabular}{|l|r|r|}
\hline & \multicolumn{1}{|c|}{ Round 1 } & \multicolumn{1}{c|}{ Round 2 } \\
\hline \# of statements & 3627 & 3640 \\
\hline \# of logical faults & 15 & 8 \\
\hline \# of faulty statements & 34 & 18 \\
\hline \# of Passed / Failed test cases & $7160 / 6425$ & $12130 / 1455$ \\
\hline \# of Passing / Failing rules & $171 / 115$ & $136 / 61$ \\
\hline \# (\%) of statements executed & 2529 & 3624 \\
& $(69.72 \%)$ & $(99.56 \%)$ \\
\hline \# of faulty statements covered & 16 & 18 \\
\hline
\end{tabular}

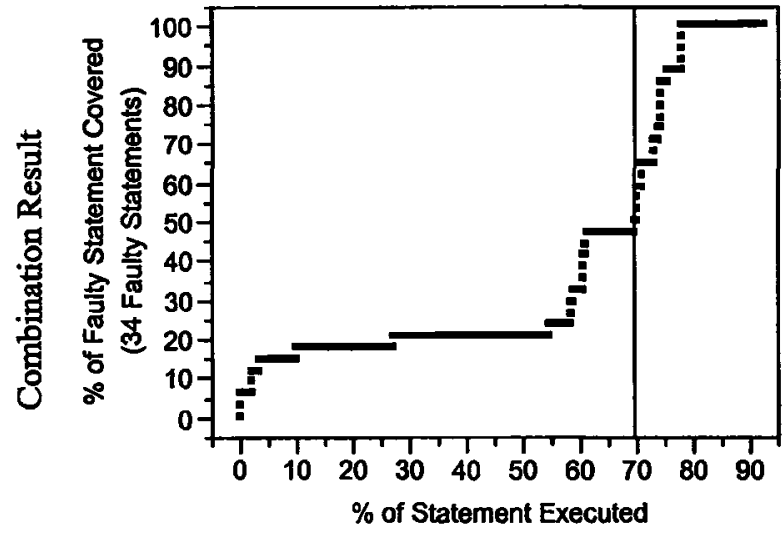

(a) Tarantula

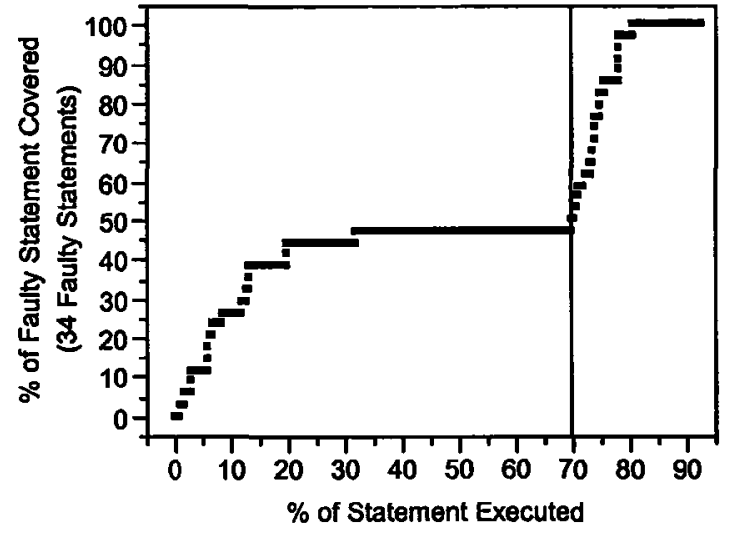

(b) RUBAR

Figure 5-2 Fixing all the faults in the statement(s) executed

In the second debugging iteration, Tarantula and RUBAR generated similar curves (the second part of the curves in Figure 5-2). But when looking into the statement rankings (Table 5-3), we find that the two methods ranked the faults in different orders. Table 5-3 has two parts. The left part shows the defects (bugs), the corresponding line number(s) the number of passing and failing test cases that execute them, and the ratio of $\left|T_{P}(s)\right| \backslash\left|T_{F}(s)\right|$. For instance, defect number 23 (Bug23), which is a fault on line number 6761, and is executed by 497 (resp. 125) failing (resp. passing) test cases. The right part if Table 5-3 shows the Tarantula and RUBAR rankings of the faulty statements listed in the left part of the table. For instance, Tarantula ranks statement 2593 before statement 2617 , before statement $2627 \ldots$ 
From the ranking result of Tarantula, we can see that the statement ranking depends on the ratio $\left|T_{P}(s)\right|\left|T_{F}(s)\right|$ (smaller values of ratio are ranked first), as we discussed in section 4.2: the first 6 statements ranked by Tarantula (Table 5-3) are those with 0 passing test cases; the remaining statements are ranked in increasing order of the ratio value. On the other hand, the ranking result of RUBAR has no direct relationship with $\left|T_{P}(s)\right| \backslash T_{F}(s) \mid$.

When further looking into the program and the location of faults Bug16, Bug22 and Bug23, the execution sequence of those faulty statements can be described as Figure 5-3 (control flow graph). The control flow graph shows that statement 6761 can potentially belong to more faulty behaviors than the other three, and that statement 7626 can potentially belong to more faulty behaviors than the remaining two. As a result, statement 6761 should be considered as the most suspicious and the most dangerous of these four statements. Therefore, it would be meaningful if the four faulty statements were ranked following their execution order, which is the ranking generated by RUBAR: statement 6761 before statement 7626 , followed by statement 3796 and statement 7627 . On the other hand, Tarantula ranked these four statements in an inverse order: e.g., statement 6761 is the last one.

We conclude that the RUBAR ranking is more acceptable and meaningful for debugging purposes than the Tarantula ranking for the Space program and its test suite. 
Table 5-3 Faulty statements and their ranks

\begin{tabular}{|l|l|r|r|r|}
\hline \multirow{2}{*}{ Defect } & \multirow{2}{*}{ Line number } & \multicolumn{3}{|c|}{ \# of test cases executed } \\
\cline { 3 - 5 } & & Fail & Pass & IT $(s) M T_{F}(s) \mid$ \\
\hline Bug13 & 2593 & 687 & 0 & 0 \\
\hline Bug13 & 2617 & 39 & 0 & 0 \\
\hline Bug13 & 2627 & 39 & 0 & 0 \\
\hline Bug16 & 3796 & 243 & 0 & 0 \\
\hline Bug23 & 6761 & 497 & 125 & 0.252 \\
\hline Bug20 & 7568 & 242 & 84 & 0.347 \\
\hline Bug22 & 7626 & 311 & 0 & 0 \\
\hline Bug22 & 7627 & 69 & 0 & 0 \\
\hline Bug07 & 8004 to 8007 & 252 & 157 & 0.623 \\
\hline Bug08 & 8269 to 8272 & 111 & 166 & 1.495 \\
\hline Bug16 & 8416 & 484 & 84 & 0.174 \\
\hline Bug18 & 8535 & 33 & 21 & 0.636 \\
\hline
\end{tabular}

\begin{tabular}{|r|r|}
\hline \multicolumn{2}{|c|}{ Rank } \\
\hline Tarantula & \multicolumn{1}{|c|}{ RUBAR } \\
\hline 2593 & 2593 \\
\hline 2617 & 6761 \\
\hline 2627 & 7626 \\
\hline 3796 & 3796 \\
\hline 7626 & 8416 \\
\hline 7627 & 7627 \\
\hline 8416 & 8004 to 8007 \\
\hline 6761 & 2617 \\
\hline 7568 & 2627 \\
\hline 8004 to 8007 & 8535 \\
\hline 8535 & 8269 to 8272 \\
\hline 8269 to 8272 & 7568 \\
\hline
\end{tabular}

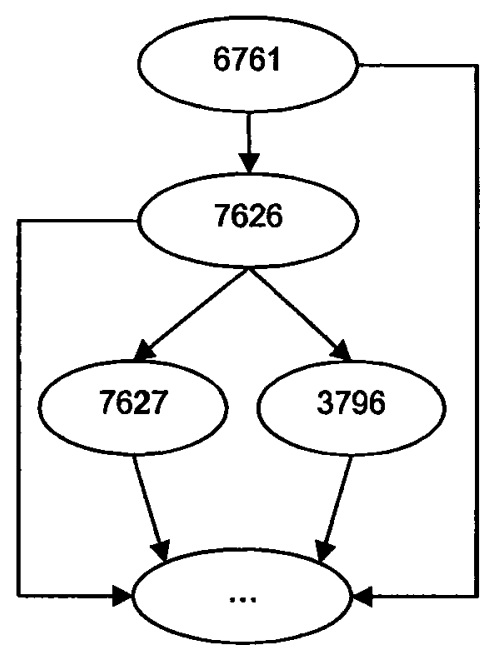

Figure 5-3 Execution sequence of faults: Bug16, Bug22 and Bug23

In general, it is very clear that when comparing Figure 5-2 (a), Figure 5-2 (b) and Table 5-3, Tarantula does not perform well since many of the faulty statements (or the most suspicious statements) seem to be assigned a higher color value and are not ranked among the first statements to be inspected. We believe, for the reasons discussed previously, that this is due to the incapacity of Tarantula to handle multiple faults in a program.

On the other hand, the technique we propose based on $\mathrm{C} 4.5$ rules performs much better 
(Figure 5-2 (b)). For each debugging iteration, RUBAR always ranks the suspicious statements first, whereas Tarantula is likely to rank some suspicious statements (the statements between 55\% and 65\% in Figure 5-2 (a)) late in the list. For RUBAR ranking, we can see a very sharp increase in the number of faulty statements covered in the first $20 \%$ ranked statements, which is roughly the range of practical interest in our situation, as discussed by Jones et al. [15].

As a side discussion, let's focus on the ranking of the (non-faulty) initialization statements of both RUBAR and Tarantula. There are around 310 initialization statements in Space program, which initialize keyword definitions, error messages etc. Since initialization statements are executed by (almost) all test cases, these initialization statements are ranked in the first debugging iteration and spotted in the first segment of both the curves in Figure 5-2. When looking into the statement ranking, we found that each method assigned a similar ranking to these initialization statements, i.e. the range of ranking values of those statements is small: $[0.4998,0.5]$ for Tarantula, [24.8547, 24.9316] for RUBAR. It is not surprising to obtain such a result since these statements are executed by (almost) all the test cases. But for Tarantula, the initialization statements are ranked in the middle (from $18 \%$ to $27 \%$ ) as Jones mentioned in their experiments [15]. On the contrary, RUBAR ranked the initialization statements near to the end (from $60 \%$ to 69\%). Since initialization statements are executed by (almost) all test cases, a fault in those statements would not change these rankings drastically. Note that we have experimented with RUBAR and Tarantula on Space with faulty initialization statements and confirmed this. This confirms that both Tarantula and RUBAR are not adequate enough for finding faulty initialization statements. 
5.2.4.2 Strategy two: fix faults in the first $20 \%$ of the ranked statements

In this second strategy, instead of fixing all the faults in the executed statements, we only fixed the faults in the first $20 \%$ of the ranked statements in the iterative process. Following this scenario, we compare the results for both RUBAR and Tarantula. Each technique leads to one specific debugging process from the original multi-fault version of Space program since not the same $20 \%$ statements are reported in the first iteration. Both the methods took three debugging processes to fix all the faults as showed in Table 5-4.

Table 5-4 Fixing faults in the first $20 \%$ of the ranked statements

\begin{tabular}{|l|r|r|r|r|r|r|}
\hline & \multicolumn{3}{|c|}{ Tarantula } & \multicolumn{3}{c|}{ RUBAR } \\
\cline { 2 - 7 } & Iteration1 & Iteration2 & Iteration3 & Iteration1 & Iteration2 & Iteration3 \\
\hline \# of Statements & 3627 & 3639 & 3645 & 3627 & 3639 & 3657 \\
\hline \# of Faulty statements & 34 & 28 & 18 & 34 & 19 & 1 \\
\hline \# of Passed / Failed Test cases & $7160 / 6425$ & $9339 / 4246$ & $12351 / 1234$ & $7160 / 6425$ & $11986 / 1599$ & $13371 / 214$ \\
\hline \# of Passing / Failing rules & -- & - & -- & $171 / 115$ & $174 / 69$ & $51 / 28$ \\
\hline \# (\%) of Statements executed & 2529 & 2837 & 3344 & 2529 & 3352 & 3367 \\
\hline $\begin{array}{l}\text { \# of Faulty statements covered } \\
\text { \# of Faulty statements covered } \\
\text { in the first 20\% statements } \\
\text { ranked }\end{array}$ & $169.72 \%)$ & $(77.96 \%)$ & $(91.72 \%)$ & $(69.72 \%)$ & $(92.11 \%)$ & $(92.07 \%)$ \\
\hline
\end{tabular}

From Table 5-4, we can see that RUBAR acts much better than Tarantula. Tarantula localized 6,10 , and 18 faulty statements in the three debugging iterations respectively, whereas RUBAR localized 15,18 and one faulty statement(s) in the three debugging iterations. Recall that the iterations focus on $20 \%$ of the ranked statements, so by visiting the same number of statements, RUBAR helps localize faults much faster. We can also draw out the similar result from the separated cost-effectiveness curves for both methods in Appendix C.3.2.

Applying the cost-effectiveness analysis on the statement rankings, we obtained Figure 5-4 for both methods with debugging iterations separated by the separation lines in the X- 
axis. Obviously, RUBAR acts much better in fault localization than Tarantula. More than $95 \%$ faulty statements are ranked in the first $30 \%$ by RUBAR. Only about $45 \%$ faulty statements are ranked in the first $30 \%$ by Tarantula. Appendix C.3.2 gives the statement ranking of each iteration.

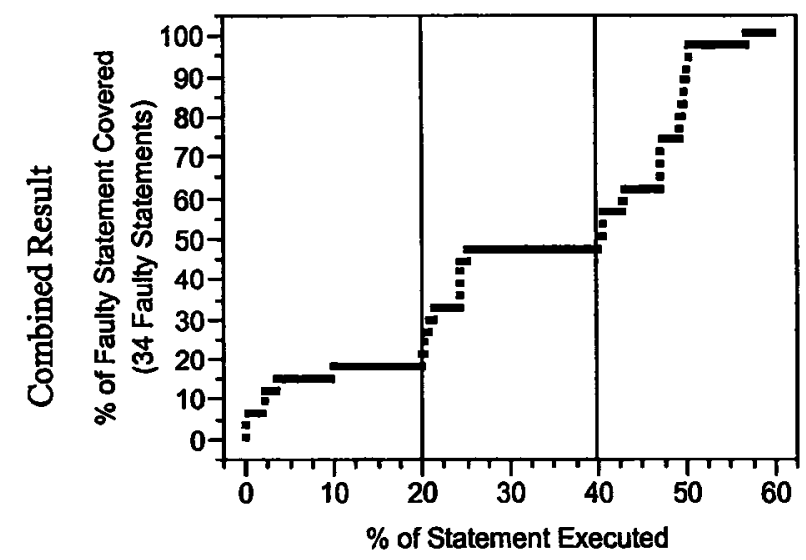

(a) Tarantula

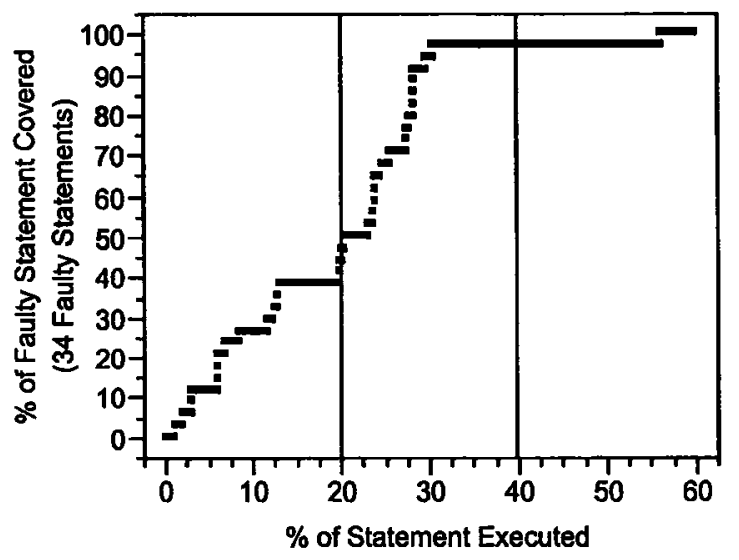

(b) RUBAR

Figure 5-4 Fixing faults in the first $20 \%$ of the ranked statements

When looking into the statement ranking of Tarantula, we found that nine of the ten faulty statements ranked low (after the first 20\%) in the first debugging iteration (Appendix C.3.2) are still ranked lower (after 20\%) in the second iteration, and are finally ranked between $9.5 \%$ and $20 \%$ in the third iteration. In the Space program, eight of these nine faulty statements (belonging to four faults) are in a method named fixsgrel () and distributed in four "switch" branches. This method will be executed by a test case that defines a grid and a rotation sequential phase. Such a test case will execute one of these four branches (according to the given grid type), but not all test cases will trigger one fault. There are only several hundred test cases (a very small percentage of the total test suite) fitting the above conditions and only a small ratio of the test cases fail. According to the Tarantula algorithm, the statements of method fixsgrel() will 
therefore obtain a high color value and be ranked very low, since they are only executed by a small portion of failed test cases: they look suspicious to Tarantula but not very suspicious. When applying the C4.5 classification algorithm, these test cases were grouped into 24 failing rules and 37 passing rules with the same input condition “Grexc_Grpha_Def $=$ ROTATION" and 10 (resp. 14) of these failing (resp. passing) rules were chosen to perform RUBAR analysis (they satisfy the rule selection criteria). As a result, these statements obtained weight values varying from -0.01 to 0.3 and ranked in the first $20 \%$ of the statements. Thanks to 44.5 rules, RUBAR is able to identify those statements more suspiciously than Tarantula. This partly explains the difference between Figure 5-4 (a) and Figure 5-4 (b): some of the statements ranked in the third part of Figure 5-4 (a) are ranked in the first part of Figure 5-4 (b).

The last faulty statement of the nine is very special. It has a low rank in the first two iterations of both methods. After looking into the code, we learnt that this fault lies in a function to initialize the variables of the port definition when no port is defined in the inputs of a test case. This function is somehow like an initialization function and is executed by most of the test cases (except those with a port definition). This is the reason why this faulty statement is ranked very low by both methods. The program version for the third iteration of RUBAR contains only this fault. In the third iteration Weka generated a $\mathrm{C} 4.5$ tree in which all the failing rules identify the failing condition above. This verified, from another perspective, the completeness and correctness of our Category-Partition definitions and the idea of using data mining rules to assist program debugging. 


\subsubsection{Diagnosis accuracy evaluation}

As discussed above (section 2.2.3), DBBs can be applied to evaluate diagnosis accuracy. We select three criteria to evaluate the accuracy of the Tarantula coloring method and our RUBAR algorithm: the number of DBBs, the average size of DBB and the ideal diagnosis accuracy (idealAccuracy $(P)$ in section 2.2.3). Figure 5-4 presents the evaluation results of the experiments we performed in section 5.2.4.1 and section 5.2.4.2. (Each time, we used the final ranking in the formulas of section 2.2.3.) From these results, RUBAR appears to be more accurate than Tarantula. With the assistance of RUBAR (resp. Tarantula) diagnosis algorithm, the average number of statements to be visited to isolate an actual fault is 25.56 (resp. 34.48) and 7.49 (resp. 11.95) for Strategy one and Strategy two respectively.

Although they generated fewer dynamic basic blocks on the second fault-fixing strategy (fixing the faults in the first $20 \%$ the ranking statements) than on the first one (fixing all the faults in the statements ranked), both diagnosis algorithms reduce the average $D B B$ size and the ideal diagnosis accuracy on the second strategy. When applying Strategy two, ideally, a programmer only needs to visit one third of the statements $(11.95 / 34.48$ with Tarantula algorithm and 7.49/25.26 with RUBAR algorithm) as they would do when using Strategy one. That is, when performing fault localization, the second fault-fixing strategy is more efficient than the first one. Just as Jones [14] concluded, the first $20 \%$ ranking statements are more fault-prone. 
Table 5-5 Diagnosis accuracy evaluation

\begin{tabular}{|l|r|r|r|r|}
\hline & \multicolumn{2}{|c|}{ Strategy one } & \multicolumn{2}{c|}{ Strategy two } \\
\cline { 2 - 5 } & Tarantula & RUBAR & Tarantula & \multicolumn{1}{c|}{ RUBAR } \\
\hline \# of DBBs & 417 & 557 & 299 & 480 \\
\hline Average size of DBBs & 8.06 & 6.03 & 7.29 & 4.55 \\
\hline Ideal diagnosis accuracy & 34.48 & 25.26 & 11.95 & 7.49 \\
\hline
\end{tabular}

\subsection{Cast study 2: the PackHexchar program}

\subsubsection{Introduction}

PackHexChar is a Java adaptation of the Sreadhex procedure, used in the GhostScript program and described in [21], to manipulate hexadecimal characters. PackHexChar [5] has three inputs: a string $S$, two integers $R L E N$ and $O D D_{-} D I G I T$. The input $S$ is a string of hexadecimal digits. The main purpose of PackHexChar is to generate a compacted representation of $S$ in binary format, specifically as an array of Bytes: e.g., string "34AB", corresponding to binary values $0011,0100,1010$, and 1011 , is compacted into an array of two Byte values 00110100 and 10101011. In the input string, characters other than hexadecimal ones are ignored. In addition to the array of Bytes, the program returns an integer value. If the input string contains an even number of hexadecimal characters, pairs of hexadecimal characters are compacted, and the returned integer value equals to 1. If the input string contains an odd number of hexadecimal characters, an even number of characters is compacted, and the program returns the remaining hexadecimal character. A user can decide to look at only a sub-string of the input string $S$, by using the input parameter RLEN: the RLEN first characters of S are analyzed and compacted. If RLEN is not a legal value (negative or greater that $S$ 's length), the program returns value -2 . The user can also ask the program to append a hexadecimal character at the beginning of $S$, by using the input parameter $O D D \_D I G I T$. This is useful when a string is split and analyzed 
in pieces with repeated calls to PackHexChar: a call can return a trailing hexadecimal character, which has to be appended at the beginning of the string during the next call. An ODD_DIGIT value of -1 indicates that no character is to be appended. If ODD_DIGIT has an illegal value (below -1 or not a hexadecimal value), the program returns value $-\mathbf{3}$.

In [5], the authors implemented the PackHexChar program in Java. They also applied the Category-Partition technique on the program and identified nine categories and 36 choices. To perform their case study, the authors generated a pool with 230 mutants of class PackHexChar by using MuJava ${ }^{10}$. These 230 mutants can be classified into 10 groups according to the mutation operators used. After applying their test suite refinement technique, they generated a test suite with 221 test cases. As a result, each mutant can be killed by at least one test case of the test suite. In this case study, we used those CP definitions, mutants and test cases to perform the experiment.

\subsubsection{A multi-fault version of PackHexChar}

When performing the cost-effectiveness analysis, instead of comparing Tarantula and RUBAR on one multi-fault program version as we did in the first case study, we made a statistical comparison using more than one multi-fault version. To obtain more comparable rankings, we ensured that each fault version contains the same number of faults. According to the size of the PackHexChar program and our intention of a balanced number ${ }^{11}$ of passing and failing test cases, we used three mutants to create each faulty version. We first randomly select 51 mutants from the mutant pool of 230 PackHexChar mutants, making sure that at least one mutant was selected for each mutant operator.

\footnotetext{
${ }^{10} \mathrm{http}: / /$ ise.gmu.edu/ ofut/mujava/

${ }^{11}$ In this case study, at least $40 \%$ and at most $60 \%$ test cases shall pass under a multi-fault version. That is, the number of passing test cases shall be larger than 88 and no more than 132 out of the 221 test cases.
} 
These 51 mutants were divided into 20 groups according to the statement they modified. That is, mutants that modify the same "correct" statement form a group. Using the 51 mutants we therefore randomly created 135 different PackHexChar program versions ${ }^{12}$, each one containing three faults, and making sure that the three faults belonged to three different mutation groups.

Last, we did not follow the iterative process discuss in section 4.3.4 since this would have been too expensive: we would have obtained 135 different series of rankings.

\subsubsection{Rule Induction Based on Category Partition}

To evaluate the effectiveness of the rule induction technique, we apply the statistics analysis mentioned in section 3.3 . In this case study, we used the $95 \%$ confidence coefficient and since the sample size is $N=135, t_{0.025,134}=1.96$.

By applying the $\mathrm{C} 4.5$ classification algorithm on the selected 135 faulty versions of PackHexChar, we obtained a classification tree for each version (Appendix D.1 are three sample C4.5 trees generated). These trees are accurate in test case classification; for example, the Fail class has an average $93.9 \%$ precision and $89.8 \%$ recall (Table $5-6$ ). The mean ROC area is 0.908 , which is excellent according to the accuracy point discussed in section 3.2.3.2.

\footnotetext{
${ }^{12}$ The original PackHexChar program was instrumented with all 51 mutants to be able to selectively enable them or not (e.g., we added control flow statements to enable them). In the results, we made sure not to rank those instrumentation statements.
} 
Table 5-6 Statistical accuracy of stratified 10-fold cross-validation by class

\begin{tabular}{|c|c|c|c|c|c|c|}
\hline \multirow[b]{2}{*}{ Class } & \multirow[b]{2}{*}{ Items } & \multirow[b]{2}{*}{ Min value } & \multirow[b]{2}{*}{ Max value } & \multirow[b]{2}{*}{ Mean value } & \multicolumn{2}{|c|}{$\mathbf{9 5 \%}$ Confidence Interval } \\
\hline & & & & & Lower limit & Upper limit \\
\hline \multirow{6}{*}{ Fail } & TN Rate & 0.789 & 1.000 & 0.898 & 0.891 & 0.905 \\
\hline & FN Rate & 0.000 & 0.226 & 0.063 & 0.058 & 0.069 \\
\hline & Precision & 0.816 & 1.000 & 0.939 & 0.932 & 0.946 \\
\hline & Recall & 0.789 & 1.000 & 0.898 & 0.891 & 0.905 \\
\hline & F-Measure & 0.815 & 0.995 & 0.916 & 0.912 & 0.919 \\
\hline & ROC Area & 0.877 & 0.995 & 0.908 & 0.903 & 0.912 \\
\hline \multirow{6}{*}{ Pass } & TP Rate & 0.774 & 1.000 & 0.937 & 0.931 & 0.942 \\
\hline & FP Rate & 0.000 & 0.211 & 0.102 & 0.095 & 0.109 \\
\hline & Precision & 0.780 & 1.000 & 0.881 & 0.872 & 0.890 \\
\hline & Recall & 0.774 & 1.000 & 0.937 & 0.931 & 0.942 \\
\hline & F-Measure & 0.831 & 0.996 & 0.906 & 0.902 & 0.910 \\
\hline & ROC Area & 0.877 & 0.995 & 0.908 & 0.903 & 0.912 \\
\hline
\end{tabular}

These classification trees are also helpful in understanding the failing condition(s) of the failing test cases. Take the fault version Ver-71 as an example, which contains mutant AODU4. A test case will kill this mutant when the test case asks for a transformation of an ODD number of hexadecimal characters. From the C4.5 tree in Appendix D.1.1, we can identify that when the input string $s$ contains an EVEN number of hexadecimal characters and the ODD_DIGIT value is not -1 (that is, a character will be appended, which makes it a transformation of an ODD number of hexadecimal characters), the test case has a large probability to fail. This is consistent with the effect of the mutant AODU4.

\subsubsection{The statistical cost-effectiveness analysis}

For this experiment, we ranked the statements on each individual faulty version with the Tarantula method and the RUBAR method respectively. To better understand the costeffectiveness of both methods, we calculate the statistical values (e.g. mean, 95\% confidence interval) of those cost-effectiveness analyses of both methods. Then, we obtained the statistical cost-effectiveness of the ranking of statements based on Tarantula 
and RUBAR as reported in Figure 5-5(a) and Figure 5-5(b). The middle curve represents the estimation of the mean cost-effectiveness, the bottom curve shows the estimated lower limit of the mean and the top curve shows the estimated upper limit of the mean.

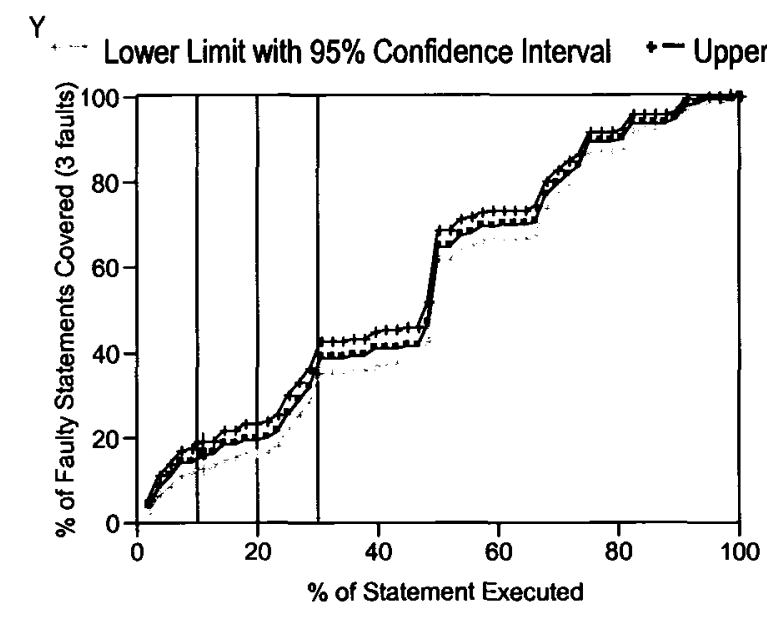

(a) Tarantula

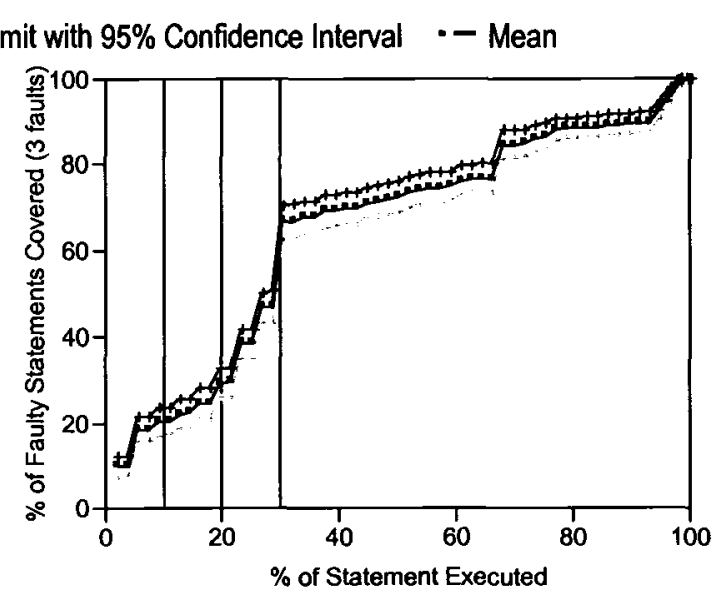

(b) RUBAR

Figure 5-5 Statistical statement ranking of 135 faulty versions

From the graph, we can see that RUBAR acts better than Tarantula. For example, the statement ranked first (the starting point of the curve) by RUBAR has a probability of $9.74 \%$ to be a faulty statement, and that statement ranked by Tarantula has a probability of $3.55 \%$ to be a faulty statement. By visiting $10 \%$ (resp. $30 \%$ ) statements, RUBAR pinpoints to $20.31 \%$ (resp. $66.48 \%$ ) faulty statements whereas Tarantula pinpoints only $15.66 \%$ (resp. $38.89 \%$ ) faulty statements. 


\section{CHAPTER 6 CONCLUSIONS AND FUTURE WORK}

\subsection{Conclusions}

This thesis describes a new strategy (RUBAR) to support debugging by ranking statements according to their likelihood of containing a fault and by identifying in a precise manner the input conditions/properties leading to failure. The general idea is to analyze test case executions using a decision-tree learning algorithm that tries to model which conditions lead to test case failure. Potentially relevant conditions/properties are predefined using a black-box test technique (Category-Partition). Each path in the tree then represents a rule modeling distinct conditions of failures, possibly originating from different faults, and leads to a distinct failure probability prediction. Then, in a way similar to the well-known Tarantula technique, this is used to perform statement ranking by accounting for the statement coverage of failing/passing test cases within each rule, assuming that test cases are likely to fail due to the same faults when belonging to the same rule. The rankings of all Pass and Fail rules is then aggregated to form a final statement ranking to be used by the tester to prioritize his or her search. To summarize, our main objectives are two-fold: (1) deal with programs containing multiple faults and (2) provide testers with precise information regarding the conditions of failure.

Two case studies were presented to demonstrate the improvements generated by our approach when compared to the original Tarantula technique. Results show that many faulty statements would be inspected much earlier when using our approach. The difference is clearly of practical significance. It also confirms, following a procedure 
different from the ones applied in literature, that iteratively looking at (and fixing defects in) the first $20 \%$ ranked statements is a reasonable heuristic to apply during fault localization.

In addition, though it is harder to demonstrate its practical value in quantitative terms, the C4.5 decision tree is also very accurate at modeling the various conditions of failures, thus helping testers understand what could be the causes of these failures. Such conditions are modeled as rules characterizing the input and output properties of test cases.

\subsection{Future work}

First, more research is required to accurately locate faults located in initialization statements. The problem is that those faults are often executed equally by failing and passing test cases, leading to those statements being ranked in the middle of the ranking. Our case studies and other researchers' experiments $[3,15]$ all show that current diagnosis algorithms cannot accurately locate those faults. Different diagnosis algorithms need to be devised.

Most of the existing fault localization algorithms are based on statement coverage. It is usually time-consuming to generate and analyze code coverage for each test case, especially when the test suite and/or the program-under-test are very large. Some research $[9,18]$ focuses on the method level fault localization, but require instrumentation and analysis of execution traces, which can be expensive and may alter (thought slightly) program behavior. Future work will investigate other techniques, such as method coverage, and compare results obtained with statement coverage. 
Another issue is the comparison with existing cluster-based fault localization techniques $[13,38]$ and the debugging-in-parallel method [13]. These methods have been proved to be efficient in a multi-fault scenario. RUBAR is also a cluster-based fault localization method and it uses $\mathrm{C} 4.5$ rules to cluster the failed test cases. Compared with $[13,38]$, RUBAR does not need the execution models to group the failed test cases and the information of the cluster (rule definitions) generated by RUBAR can also help program diagnosis.

Future work should also include additional case studies, and the application of similar ideas to regression testing and software evolution. 


\section{CHAPTER 7 REFERENCE}

[1] Agrawal H., Horgan J. R., London S., et al., "Fault Localization using Execution Slices and Dataflow Tests," International Symposium on Software Reliability Engineering, Toulouse, France, 1995.

[2] Araki K., Furukawa Z., and Cheng J., "A General Framwork for Debugging," IEEE Software, 1991.

[3] Baudry B., Fleurey F., and Traon Y. L., "Inproving Test Suites for Efficient Fault Localization," International Conference on Software Engineering, Shanghai, China, 2006.

[4] Bowring J. F., Rehg J. M., and Harrold M. J., "Active Learning for Automatic Classification of Software Behavior," Proceding of the International Symposium on Software Testing and Analysis, Boston, Massachusetts, USA, 2004.

[5] Briand L. C., Labiche Y., and Bawar Z., "Using Machine Learning to Refine Blackbox Test Specifications and Test Suites," Software Quality Engineering Laboratory, Carleton University TR SCE-07-05, 2007.

[6] Brun Y. and Ernst M. D., "Finding latent code errors via machine learning over program executions," International Conference on Software Engineering, 2004.

[7] Cleve H. and Zeller A., "Locating Causes of Program Failures," Proceeding of International Conference on Software Engineering, St. Louis, Missouri, USA, 2005.

[8] Cohen W. W. and Singer Y., "Simple, Fast, and Effective Rule Learner," AAAI/IAAI, 1999.

[9] Dallmeier V., Lindig C., and Zeller A., "Lightweight Defect Localization for Java," European Conference on Object-Oriented Programming, Glasgow, Scotland, 2005.

[10] Demillo R. A., Pan H., and Spafford E. H., "Cirtical Slicing for Software Fault Localization," ISSTA, San Diego, California, U.S.A., 1996.

[11] Emst M. D., Cockrell J., Griswold W. G, et al., "Dynamically discovering likely program invariants to support program evolution," IEEE Transaction on Software Engineering, vol. 27, pp. 1-25, 2001.

[12] Hutchins M., Froster H., Goradia T., et al., "Experiments on the Effectiveness of Dataflow- and Controlflow-Based Test Adequacy Criteria," $16^{\text {th }}$ IEEE International Conference on Software Engineering, Sorrento (Italy), 1994.

[13] Jones J. A., Bowring J. F., and Harrold M. J., "Debugging in Parallel," ISSTA, London, England, 2007.

[14] Jones J. A. and Harrold M. J., "Empirical Evaluation of the Tarantula Automatic Fault-Localization Technique," International Conference on Automated Software Engineering, Long Beach, California, 2005. 
[15] Jones J. A., Harrold M. J., and Stasko J. T., "Visualization of Test Information to Assist Fault Localization," International Conference on Software Engineering, Orlando, Florida, 2002.

[16] Jorgensen P. C., Software Testing: A Craftsman's Approach, $2^{\text {nd }}$ ed: CRC Press, 1995.

[17] Liblit B., Naik M., Zheng A. X., et al., "Scalable Statistical Bug Isolation," $A C M$ SIGPLAN conference on Programming language design and implementation, 2005.

[18] Liu C., "Mining Control Flow Abnormality for Logic Error Isolation," SIAM International Conference on data Mining, 2006.

[19] Liu C. and Han J., "Failure Proximity: A Fault Localization-Based Approach," ACM SIGSOFT Foundations of Software Engineering, 2006.

[20] Myers G. J., The art of Software Testing, $2^{\text {nd }}$ ed: Hohn Wiley \& Sones, Inc, 1979.

[21] Ostrand T. J. and Balcer M. J., "The Category-Partition Method for Specifying and Generating Functional Test," Communications of the ACM, vol. 31, pp. 676-686, 1988.

[22] Pan H. and Spafford E. H., "Heuristics for Automatic Localization of Software Faults," Purdue Universtiy Technical Report SERC-TR-116-P, 1992.

[23] Pasquini A., Crespo A., and Matrelle P., "Sensitivity of reliability-growth models to operational profiles errors vs testing accuracy," IEEE Transactions on Reliability, vol. 45, pp. 531-540, 1996.

[24] Podgurski A., Leon D., Francis P., et al., "Automated Support for Classifying Software Failure Reports," International Conference on Software Engineering, 2003.

[25] Pressman R. S., Software Engineering: A Practitioner's Approach, $5^{\text {th }}$ ed: McGrawHill Companies, Inc, 2001.

[26] Quinlan J. R., C4.5: Programs for Machine Learning: Morgan Kaufmann, 1993.

[27] Renieris M. and Reiss S. P., "Fault Localization with Nearest Neighbor Queries," International Conference on Automated Software Engineering, Montreal, Quebec, 2003.

[28] Rothermel G., Untch R. H., Chu C., et al., "Prioritizing test cases for regression testing," IEEE Transactions on Software Engineering, vol. 27, pp. 929-948, 2001.

[29] Snedecor G. W. and Cochran W. G., Statistical Methods, Eighth ed: Iowa State University Press, 1989.

[30] Vokolos F. I. and Frankl P. G., "Empirical evaluation of the textual differencing regression testing technique," IEEE International Conference on Software Maintenance, Bethesda, MD, USA, 1998.

[31] Weiser M., "Program slicing," IEEE Transactions on Software Engineering, pp. 10:352--357, 1984. 
[32] Weiser M., "Programmers use slices when debugging," Communications of the $A C M$, vol. 25, pp. 446-452, 1982.

[33] Witten I. H. and Frank E., Data Mining: Practical Machine Learning Tools and Techniques, $2^{\text {nd }}$ ed: Morgan Kaufman, 2005.

[34] Wong W. E., Horgan J. R., Mathur A. P., et al., "Test Set Size Minimization and Fault Detection Effectiveness: A Case Study in a Space Application," Software Engineering Research Center (SERC), Technical Report TR-173-P, 1997.

[35] Xu S. and Rajlich V., "Cognitive Process during Program Debugging," the IEEE International Conference on Cognitive Informatics, Irvine, California, U.S.A., 2004.

[36] Yoon B. and Garcia O. N., "Cognitive activities and support in debugging," Proceedings of Annual Symposia on Human Interaction with Complex System, 1998.

[37] Zeller A., Why Programs Fail: A guide to Systematic Debugging: Morgan Kaufman, 2005.

[38] Zheng A. X., Jordan M. I., Liblit B., et al., "Statistical Debugging: Simultaneous Identification of Multiple Bugs," Proceddings of International Conference on Machine Learning, Pittsburgh, PA, 2006. 


\section{APPENDIX A TRIANGLE PROGRAM}

The triangle program accepts three integers as inputs, which are taken as the sides of a triangle. The output of this program is the type of the triangle determined by the three sides: Equilateral, Isosceles, Scalene, or NotATriangle.

Table A-1 describes the inputs of ten test cases and the type of triangle determined by these inputs. Suppose that the triangle program has bugs to detect NotATriangle when the sizes are illegal (Figure A-1). The test suite will generate a test result in Table A-1 (the last column).

As mentioned above, the category-partition method can be used to identify the inputs of a program. Table $3-1$ is a simple sample of the $\mathrm{CP}$ definition for the triangle program. When applied the CP definition of Table 3-1, the test suite of Table A-1 can be described as Table A-2.

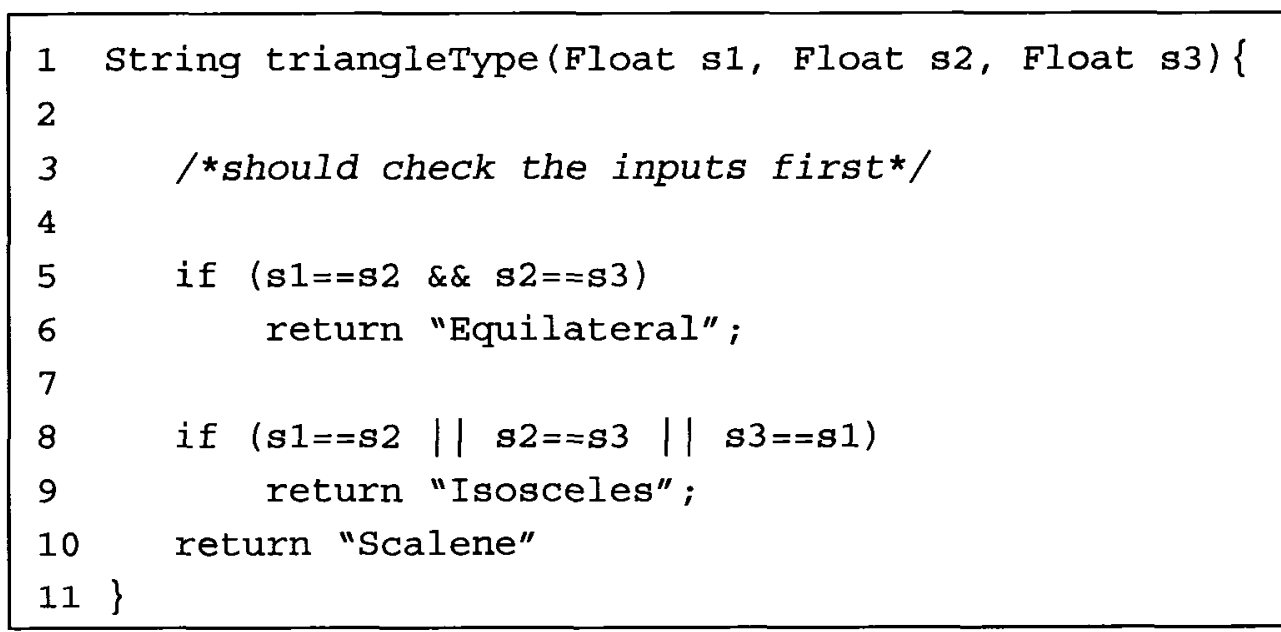

Figure A-1 Sample faulty Triangle program 
Table A-1 A test suite and the test result of the triangle program

\begin{tabular}{|c|c|c|c|c|c|}
\hline $\mathrm{TC}$ & $\mathrm{S}_{1}$ & $\mathrm{~S}_{2}$ & $\mathrm{~S}_{3}$ & Expected Result & Test Result \\
\hline $\mathrm{TC}_{1}$ & 2 & 4 & 5 & Scalene & Pass \\
\hline $\mathrm{TC}_{2}$ & 8 & 10 & 6 & Scalene & Pass \\
\hline $\mathrm{TC}_{3}$ & 6 & 6 & 6 & Equilateral & Pass \\
\hline $\mathrm{TC}_{4}$ & 6 & 5 & 5 & Isosceles & Pass \\
\hline $\mathrm{TC}_{5}$ & 11 & 11 & 3 & Isosceles & Pass \\
\hline $\mathrm{TC}_{6}$ & 3 & 2 & 3 & Isosceles & Pass \\
\hline $\mathrm{TC}_{7}$ & 1 & 2 & 3 & NotATriangle & Fail \\
\hline $\mathrm{TC}_{8}$ & 2 & 5 & 2 & NotATriangle & Fail \\
\hline $\mathrm{TC}_{9}$ & 0 & 2 & 2 & NotATriangle & Fail \\
\hline $\mathrm{TC}_{10}$ & -1 & -1 & -1 & NotATriangle & Fail \\
\hline
\end{tabular}

Table A-2 The test suite described by the CP definition

\begin{tabular}{|c|c|c|c|c|c|c|c|c|c|c|c|}
\hline $\mathrm{TC}$ & $\mathrm{CA01}$ & $\mathrm{CA02}$ & $\mathrm{CA03}$ & CA04 & CA05 & CA06 & CA07 & CA08 & CA09 & CA10 & Test Result \\
\hline $\mathrm{TC}_{1}$ & $\mathrm{~s} 1<\mathrm{s} 2$ & $\mathrm{~s} 2<\mathrm{s} 3$ & $\mathrm{~s} 3>\mathrm{s} 1$ & Yes & Yes & Yes & Yes & Yes & Yes & Yes & Pass \\
\hline $\mathrm{TC}_{2}$ & $\mathrm{~s} 1<\mathrm{s} 2$ & $\mathrm{~s} 2>\mathrm{s} 3$ & $\mathrm{~s} 3<\mathrm{s} 1$ & Yes & Yes & Yes & Yes & Yes & Yes & Yes & Pass \\
\hline $\mathrm{TC}_{3}$ & $\mathrm{~s} 1=\mathrm{s} 2$ & $\mathrm{~s} 2=\mathrm{s} 3$ & $\mathrm{~s} 3=\mathrm{s} 1$ & Yes & Yes & Yes & Yes & Yes & Yes & Yes & Pass \\
\hline $\mathrm{TC}_{4}$ & $\mathrm{~s} 1>\mathrm{s} 2$ & $\mathrm{~s} 2=\mathrm{s} 3$ & $\mathrm{~s} 3<\mathrm{s} 1$ & Yes & Yes & Yes & Yes & Yes & Yes & Yes & Pass \\
\hline $\mathrm{TC}_{5}$ & $\mathrm{~s} 1=\mathrm{s} 2$ & $\mathrm{~s} 2>\mathrm{s} 3$ & $\mathrm{~s} 3<\mathrm{s} 1$ & Yes & Yes & Yes & Yes & Yes & Yes & Yes & Pass \\
\hline $\mathrm{TC}_{6}$ & $\mathrm{~s} 1>\mathrm{s} 2$ & $\mathrm{~s} 2<\mathrm{s} 3$ & $\mathrm{~s} 3=\mathrm{s} 1$ & Yes & Yes & Yes & Yes & Yes & Yes & Yes & Pass \\
\hline $\mathrm{TC}_{7}$ & $\mathrm{~s} 1<\mathrm{s} 2$ & $\mathrm{~s} 2<\mathrm{s} 3$ & $\mathrm{~s} 3>\mathrm{s} 1$ & Yes & Yes & Yes & No & No & No & Yes & Fail \\
\hline $\mathrm{TC}_{8}$ & $\mathrm{~s} 1<\mathrm{s} 2$ & $\mathrm{~s} 2>\mathrm{s} 3$ & $\mathrm{~s} 3=\mathrm{s} 1$ & No & Yes & No & Yes & No & Yes & No & Fail \\
\hline $\mathrm{TC}_{9}$ & $\mathrm{~s} 1<\mathrm{s} 2$ & $\mathrm{~s} 2=\mathrm{s} 3$ & $\mathrm{~s} 3>\mathrm{s} 1$ & Yes & Yes & No & No & No & No & No & Fail \\
\hline $\mathrm{TC}_{10}$ & $\mathrm{~s} 1=\mathrm{s} 2$ & $\mathrm{~s} 2=\mathrm{s} 3$ & $\mathrm{~s} 3=\mathrm{s} 1$ & No & No & No & No & No & No & No & Fail \\
\hline
\end{tabular}




\section{APPENDIX B CLASS DIAGRAM OF RUBAR ALGORITHM}

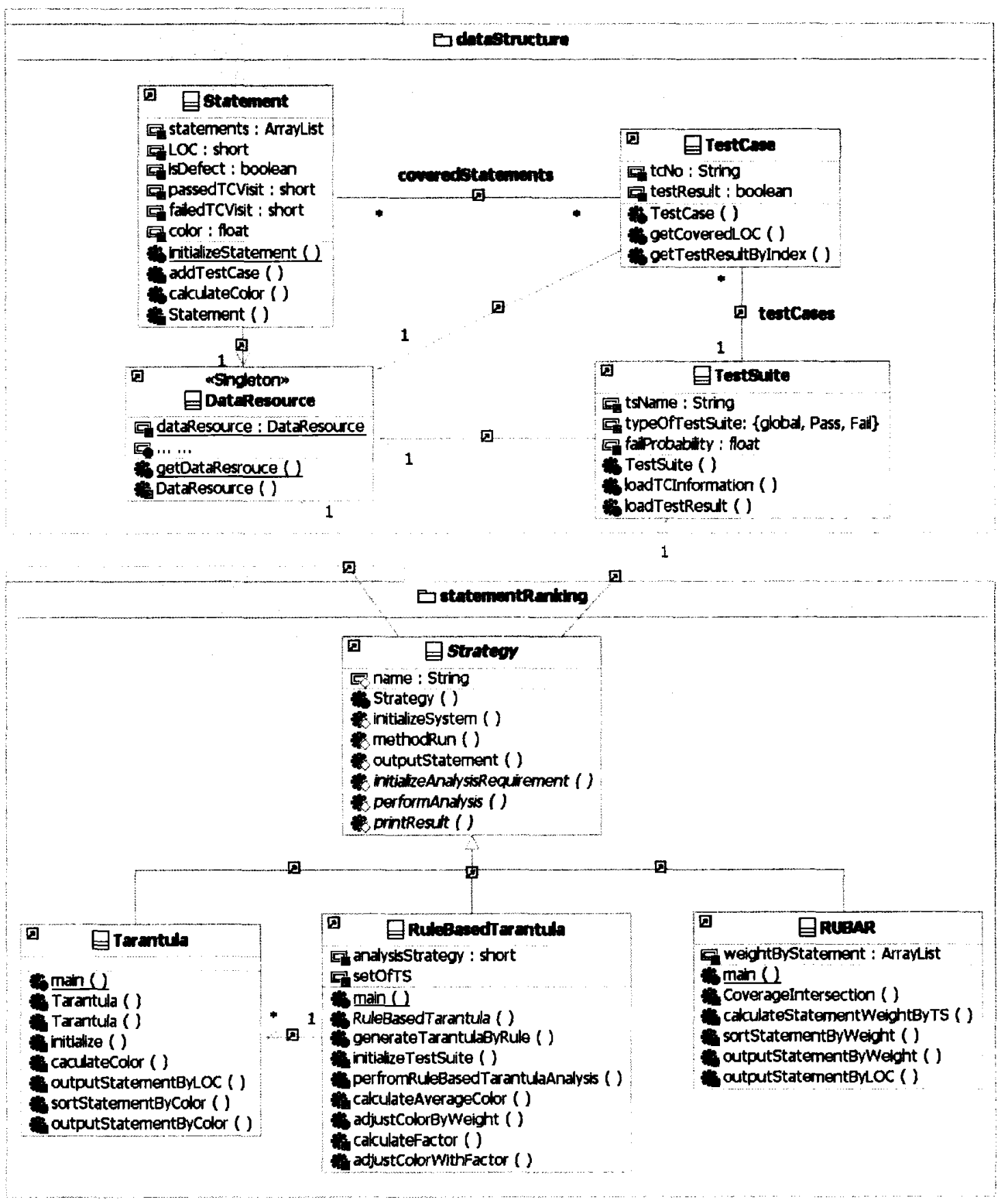




\section{APPENDIX C SPACE PROGRAM}

\section{C.1 The Space program}

The following table is a brief classification of the mutations of the SPACE program.

\begin{tabular}{|l|l|}
\hline Equivalent mutations & mutant01, mutant02, mutant32 \\
\hline Initialization mutations & mutant29, mutant34 \\
\hline Selected mutations & $\begin{array}{l}\text { mutant07, mutant08, mutant11, mutant13, mutant14, mutant15, mutant16, } \\
\text { mutant17, mutant18, mutant20, mutant22, mutant23, mutant24, mutant31, } \\
\text { mutant37 }\end{array}$ \\
\hline
\end{tabular}

\section{C.2 Category partition definition}

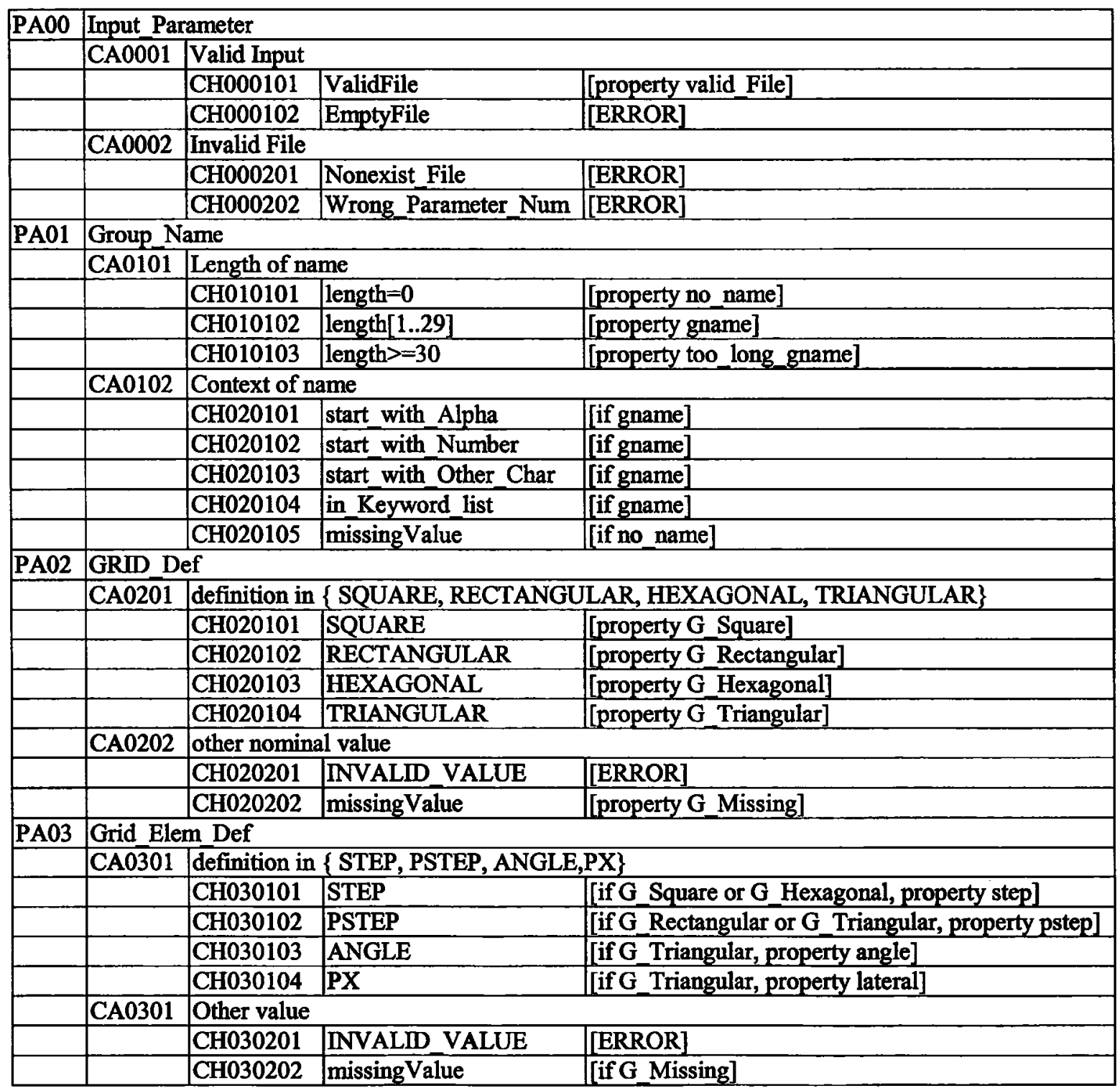




\begin{tabular}{|c|c|c|c|c|}
\hline \multicolumn{5}{|c|}{\begin{tabular}{l|l} 
PA04 & Grid Elem Vall
\end{tabular}} \\
\hline & \multicolumn{4}{|c|}{\begin{tabular}{|l|l|} 
CA0401 valid value \\
\end{tabular}} \\
\hline & & CH040101 & $=0$ & [if step or pstep or angle] \\
\hline & & CH040102 & $>0$ & [if step or pstep or angle] \\
\hline & & CH040103 & missingValue & [if G_Missing or lateral] \\
\hline & CA0402 & invalid value & & \\
\hline & & CH040201 & $<0$ & [ERROR][if step or pstep or angle] \\
\hline & & CH040202 & non-number & [ERROR][if step or pstep or angle] \\
\hline & & CH040203 & ErrorMissing & [ERROR][if step or pstep or angle] \\
\hline \multirow[t]{9}{*}{ PA05 } & \multicolumn{4}{|c|}{ Grid Elem Val2 } \\
\hline & \multicolumn{4}{|c|}{ CA0501 valid value } \\
\hline & & $\mathrm{CH} 050101$ & $=0$ & [if pstep or angle] \\
\hline & & CH050102 & $>0$ & [if pstep or angle] \\
\hline & & CH050103 & missingValue & [if step or lateral] \\
\hline & CA0502 & invalid valu & & \\
\hline & & CH050201 & $<0$ & [ERROR][if pstep or angle] \\
\hline & & СH050202 & non-number & [ERROR][if pstep or angle] \\
\hline & & $\mathrm{CH} 050203$ & ErrorMissing & [ERROR][if G Square or lateral] \\
\hline \multirow[t]{9}{*}{ PA06 } & \multicolumn{4}{|c|}{ Grid_Elem_Triang_Vall } \\
\hline & \multicolumn{4}{|c|}{ CA0601 number } \\
\hline & & CH060101 & $=\mathbf{0}$ & [if angle or literal] \\
\hline & & CH060102 & $>0$ & [if angle or literal] \\
\hline & & CH060103 & $<0$ & [if angle or literal] \\
\hline & & CH060104 & missingValue & [if not (angle or literal)] \\
\hline & CA0602 & non number & & \\
\hline & & СH060201 & non-number & [ERROR][if angle or literal] \\
\hline & & CH060202 & ErrorMissing & [ERROR][if angle or literal] \\
\hline \multirow{9}{*}{ PA07 } & \multicolumn{4}{|c|}{ Grid Elem Triang Val2 } \\
\hline & \multicolumn{4}{|c|}{\begin{tabular}{|l|l} 
CA0701 number \\
\end{tabular}} \\
\hline & & CH070101 & $=0$ & [if literal] \\
\hline & & CH070102 & $>0$ & [if literal] \\
\hline & & CH070103 & $<0$ & [if literal] \\
\hline & & CH070104 & missingValue & [if not literal] \\
\hline & CA0702 & non number & & \\
\hline & & CH070201 & non-number & [ERROR][if literal] \\
\hline & & CH070202 & ErrorMissing & [ERROR][if literal] \\
\hline \multirow[t]{9}{*}{ PA08 } & \multicolumn{4}{|c|}{ Grid_Elem_Triang_Val3 } \\
\hline & \multicolumn{4}{|c|}{ CA0801 number } \\
\hline & & CH080101 & $=0$ & [if literal] \\
\hline & & CH080102 & $>0$ & [if literal] \\
\hline & & CH080103 & $<0$ & [if literal] \\
\hline & & CH080104 & missingValue & [if not literal] \\
\hline & CA0802 & non number & & \\
\hline & & CH080201 & non-number & [ERROR][if literal] \\
\hline & & CH080202 & ErrorMissing & [ERROR][if literal] \\
\hline \multirow[t]{8}{*}{ PA09 } & \multicolumn{4}{|c|}{ Grid Elem Triang_Val3 } \\
\hline & CA0901 & number & & \\
\hline & & CH090101 & $=0$ & [if literal] \\
\hline & & CH090102 & $>0$ & [if literal] \\
\hline & & CH090103 & $<0$ & [if literal] \\
\hline & & CH090104 & missingValue & [if not literal] \\
\hline & CA0902 & non number & & \\
\hline & & CH090201 & non-number & [ERROR][if literal] \\
\hline
\end{tabular}




\begin{tabular}{|c|c|c|c|c|}
\hline & & CH090202 & ErrorMissing & [ERROR][if literal] \\
\hline \multirow[t]{9}{*}{ PA10 } & \multicolumn{4}{|c|}{ Grid_Elem_Unit } \\
\hline & CA1001 & \multicolumn{3}{|c|}{ Definition in $\{\mathrm{dm}, \mathrm{cm}, \mathrm{mm}, \mathrm{m}\}$} \\
\hline & & CH100101 & $\mathrm{dm}$ & [if step or pstep] \\
\hline & & CH100102 & $\mathrm{cm}$ & [if step or pstep] \\
\hline & & $\mathrm{CH} 100103$ & $\mathrm{~mm}$ & [if step or pstep] \\
\hline & & CH100104 & $\mathbf{m}$ & [if step or pstep] \\
\hline & CA1002 & \multicolumn{3}{|l|}{ Other value } \\
\hline & & CH100201 & INVALID VALUE & [if step or pstep] \\
\hline & & \multicolumn{3}{|c|}{\begin{tabular}{l|l} 
CH100202 missingValue \\
Angle Unit
\end{tabular}} \\
\hline \multirow[t]{7}{*}{ PA11 } & \multirow{2}{*}{\multicolumn{4}{|c|}{\begin{tabular}{|l|l|} 
Grid Elem_Angle_Unit \\
CA1101 Definition in $\{$ deg, rad $\}$
\end{tabular}}} \\
\hline & & & & \\
\hline & & \multicolumn{3}{|c|}{\begin{tabular}{|l|l|l} 
CH1 10101 & deg \\
\end{tabular}} \\
\hline & & CH110102 & $\mathrm{rad}$ & [if angle] \\
\hline & CA1102 & \multicolumn{3}{|l|}{ Other value } \\
\hline & & CH110201 & INVALID_VALUE & [if angle] \\
\hline & & CH110202 & missingValue & \\
\hline \multirow[t]{12}{*}{ PA12 } & Element & \multicolumn{3}{|l|}{ Name } \\
\hline & CA1201 & \multicolumn{3}{|c|}{ Definition in \{GEOMETRY\} } \\
\hline & & CH120101 & GEOMETRY & [property geometry] \\
\hline & CA1202 & \multicolumn{3}{|c|}{ length of string } \\
\hline & & CH120201 & length $=0$ & [if not geometry, property missing Name] \\
\hline & & CH120202 & length $[1 . .29]$ & [if not geometry, property E_Name] \\
\hline & & CH120203 & length $>=30$ & [if not geometry, property long Name] \\
\hline & CA1203 & \multicolumn{3}{|c|}{ context of name } \\
\hline & & CH120301 & start_with_Alpha & [if E Name] \\
\hline & & CH120302 & start with Number & [if E Name] \\
\hline & & CH120303 & start with_Other_Char & [if E_Name] \\
\hline & & CH120304 & in_Keyword_list & [if E_Name] \\
\hline \multirow[t]{7}{*}{ PA13 } & \multicolumn{4}{|c|}{ Elem_Geometry Def } \\
\hline & CA1301 & \multicolumn{3}{|c|}{ Definition in \{CIRCULAR, RECTANGULAR } \\
\hline & & CH130101 & CIRCULAR & [if geometry, property E_Circular] \\
\hline & & CH130102 & RECTANGULAR & if geometry, property E Rectangular] \\
\hline & CA1302 & Other value & & \\
\hline & & CH130201 & INVALID_VALUE & [if geometry, property wrong_E_Def] \\
\hline & & CH130202 & missingValue & if missing_Name] \\
\hline \multirow[t]{8}{*}{ PA14 } & \multicolumn{4}{|c|}{ Elem Geometry Val1 } \\
\hline & CA1401 & \multicolumn{3}{|c|}{ unsigned real number } \\
\hline & & CH140101 & $=0$ & [if E Circular or E Rectangular] \\
\hline & & CH140102 & $>0$ & [if E Circular or E Rectangular] \\
\hline & CA1402 & wrong value & & \\
\hline & & CH140201 & $<0$ & [ERROR] [if $\mathrm{E} \cdot$ Circular or $\mathrm{E}$ _Rectangular] \\
\hline & & CH140202 & non-number & [ERROR][if E Circular or E Rectangular] \\
\hline & & CH140203 & missingValue & [if wrong_E_Def or not geometry] \\
\hline PA15 & Elem_Ge & eometry_Val2 & & \\
\hline & CA1501 & unsigned re: & 1 number & \\
\hline & & CH150101 & $=0$ & [if E Rectangular] \\
\hline & & CH150102 & $>0$ & [if E_Rectangular] \\
\hline & CA1502 & wrong value & & \\
\hline & & CH150201 & $<0$ & [ERROR][if E_Rectangular] \\
\hline & & CH150202 & non-number & [ERROR][if E Rectangular] \\
\hline & & CH150203 & ErrorMissing & [ERROR][if E Rectangular] \\
\hline & & CH150204 & missingValue & [if not E Rectangular] \\
\hline
\end{tabular}




\begin{tabular}{|c|c|c|c|c|}
\hline A16 & Elem_Ge & eometry_Unit & & \\
\hline & CA1601 & Definition in & $\{\mathrm{dm}, \mathrm{cm}, \mathrm{mm}, \mathrm{m}\}$ & \\
\hline & & CH160101 & $\mathrm{dm}$ & [if E_Circular or E_Rectangular] \\
\hline & & CH160102 & $\mathbf{c m}$ & [if E Circular or E Rectangular] \\
\hline & & CH160103 & $\mathrm{mm}$ & [if E_Circular or E Rectangular] \\
\hline & & CH160104 & $\mathrm{m}$ & [if E Circular or E Rectangular] \\
\hline & CA1602 & Other value & & \\
\hline & & CH160201 & INVALID VALUE & [ERROR][ifE_Circular or E_Rectangular] \\
\hline & & CH160202 & missingValue & \\
\hline PA17 & Elem Po & olarization $\mathrm{Sp}$ & & \\
\hline & CA1701 & Definition ir & a LINEAR, CIRCUL & \\
\hline & & CH170101 & LINEAR & [if geometry, property E P linear] \\
\hline & & CH170102 & CIRCULAR & [if geometry, property E P circular] \\
\hline & CA1702 & Other value & & \\
\hline & & CH170201 & INVALID_VALUE & [ERROR] \\
\hline & & CH170202 & missingValue & \\
\hline PA18 & Elem_Po & lar_Def & & \\
\hline & CA1801 & Definition in & n \{ORIENTATION, L\} & H \\
\hline & & CH180101 & ORIENTATION & [if E P linear, property E P orientation] \\
\hline & & CH180102 & LH & [if E_P_circular] \\
\hline & & CH180103 & RH & [if E_P_circular] \\
\hline & CA1802 & Other value & & \\
\hline & & CH180201 & |NVALID VALUE & [ERROR][if E P linear or E P circular] \\
\hline & & CH180202 & missingValue & \\
\hline PA19 & Elem Po & olar_LinePol & Val & \\
\hline & CA1901 & Definition ir & $n\{X, Y\}$ & \\
\hline & & CH190101 & $\mathrm{x}$ & [if E P orientation] \\
\hline & & CH190102 & $\mathbf{Y}$ & [if E P orientation] \\
\hline & CA1902 & real number & & \\
\hline & & CH190201 & $=0$ & [if E P orientation] \\
\hline & & CH190202 & $>0$ & [if E P orientation] \\
\hline & & CH190203 & $<0$ & [if E P linear] \\
\hline & CA1903 & Other value & & \\
\hline & & CH190301 & INVALD VALUE & [ERROR][if E P orientation] \\
\hline & & CH190302 & errorMissing & [ERROR][if E P orientation] \\
\hline & & CH190302 & missingValue & \\
\hline PA20 & Elem Po & olar_LinePol & Unit & \\
\hline & CA2001 & Definition in & $n\{\mathrm{deg}, \mathrm{rad}\}$ & \\
\hline & & CH200101 & deg & [if E_P_orientation] \\
\hline & & CH200102 & $\mathrm{rad}$ & [if E P orientation] \\
\hline & CA2002 & null value & & \\
\hline & & CH200201 & INVALID_VALUE & ][ERROR][if $\mathrm{E}$ P orientation] \\
\hline & & $\mathrm{CH} 200202$ & missingValue & \\
\hline PA21 & Elem Po & ort Number & & \\
\hline & CA2101 & Natural num & nber & \\
\hline & & CH210101 & $=0$ & [if geometry, property no_Port] \\
\hline & & CH210102 & {$[1,998]$} & [if geometry, property medium_Size_Port] \\
\hline & & CH210103 & $=999$ & [if geometry, property large Size Port] \\
\hline & CA2102 & other numbe & & \\
\hline & & $\mathrm{CH} 210201$ & $>999$ & ][ERROR][ if geometry] \\
\hline & & CH210202 & $<0$ & [ERROR][ if geometry] \\
\hline & & CH210203 & real_number & [ERROR][ if geometry] \\
\hline & CA2103 & other value & & \\
\hline
\end{tabular}




\begin{tabular}{|c|c|c|c|c|}
\hline & & CH210301 & INVALID VALUE & [ERROR][ if geometry] \\
\hline & & $\mathrm{CH} 210302$ & missingValue & \\
\hline \multirow[t]{5}{*}{ PA22 } & \multicolumn{4}{|c|}{ Elem Port_Set_ENum } \\
\hline & \multicolumn{4}{|c|}{ CA2201 number of the element } \\
\hline & & CH220101 & $=0$ & [if no Port, property missingPortDef] \\
\hline & & CH220102 & $=1$ & [if medium_Size_Port, property onePortDef] \\
\hline & & CH220103 & $>1$ & [if medium Size Port or large_Size_Port] \\
\hline \multirow[t]{11}{*}{ PA23 } & \multicolumn{4}{|c|}{ Elem Port_Orient_Val } \\
\hline & CA2301 & \multicolumn{3}{|c|}{ content of the set } \\
\hline & & CH230101 & $\mathbf{X}$ & [if not missingPortDef] \\
\hline & & CH230102 & $\mathbf{Y}$ & [if not missingPortDef] \\
\hline & & CH230103 & $>0$ & [if not missingPortDef] \\
\hline & & CH230104 & $=0$ & [if not missingPortDef] \\
\hline & & CH230105 & $<0$ & [if not missingPortDef] \\
\hline & & CH230106 & combination & [if not (missingPortDef or onePortDef)] \\
\hline & & CH230107 & ContainInvalid & [if not (missingPortDef or onePortDef)] \\
\hline & & CH230108 & allinvalid & [if not missingPortDef] \\
\hline & & CH230109 & missingValue & \\
\hline \multirow[t]{8}{*}{ PA24 } & \multicolumn{4}{|c|}{ Elem_Port Orient_UNIT } \\
\hline & CA2401 & \multicolumn{3}{|c|}{ content of the set } \\
\hline & & CH240101 & deg & [if not missingPortDef] \\
\hline & & CH240102 & $\mathrm{rad}$ & [if not missingPortDef] \\
\hline & & CH240103 & combination & [if not (missingPortDef or onePortDef)] \\
\hline & & $\mathrm{CH} 240104$ & mixedValue & [if not (missingPortDef or onePortDef)] \\
\hline & & $\mathrm{CH} 240105$ & allinvalid & [if not missingPortDef] \\
\hline & & CH240106 & missingValue & \\
\hline \multirow[t]{9}{*}{ PA25 } & \multicolumn{4}{|c|}{ Elem Port Amp Val } \\
\hline & CA2501 & \multicolumn{3}{|c|}{ content of the set } \\
\hline & & CH250101 & $>0$ & [if not missingPortDef] \\
\hline & & CH250102 & $=0$ & [if not missingPortDef] \\
\hline & & CH250103 & $<0$ & [if not missingPortDef] \\
\hline & & CH250104 & combination & [if not (missingPortDef or onePortDef)] \\
\hline & & CH250105 & ContainInvalid & [if not (missingPortDef or onePortDef)] \\
\hline & & CH250106 & allinvalid & [if not missingPortDef] \\
\hline & & CH250107 & missingValue & \\
\hline \multirow[t]{13}{*}{ PA26 } & \multicolumn{4}{|c|}{ Elem Port Amp Unit } \\
\hline & CA2601 & \multicolumn{3}{|c|}{ content of the set } \\
\hline & & CH260101 & $\mathrm{dm}$ & [if not missingPortDef] \\
\hline & & CH260102 & $\mathbf{m m}$ & [if not missingPortDef] \\
\hline & & CH260103 & $\mathrm{cm}$ & [if not missingPortDef] \\
\hline & & CH260104 & $\mathbf{m}$ & [if not missingPortDef] \\
\hline & & CH260105 & $\mathrm{dB}$ & [if not missingPortDef] \\
\hline & & CH260106 & POWER & [if not missingPortDef] \\
\hline & & CH260107 & LINEAR & [if not missingPortDef] \\
\hline & & CH260108 & combination & [if not (missingPortDef or onePortDef)] \\
\hline & & CH260109 & ContainInvalid & [if not (missingPortDef or onePortDef)] \\
\hline & & CH260110 & allinvalid & [if not missingPortDef] \\
\hline & & CH260111 & missingValue & \\
\hline PA27 & \multicolumn{4}{|c|}{ Elem_Port_Shdef_Val } \\
\hline & CA2701 & \multicolumn{3}{|c|}{ content of the set } \\
\hline & & CH270101 & $>0$ & [if not missingPortDef] \\
\hline & & CH270102 & $=0$ & [if not missingPortDef] \\
\hline & & CH270103 & $<0$ & [if not missingPortDef] \\
\hline
\end{tabular}




\begin{tabular}{|c|c|c|c|c|}
\hline & & CH270104 & combination & [if not (missingPortDef or onePortDef)] \\
\hline & & CH270105 & ContainInvalid & [if not (missingPortDef or onePortDef)] \\
\hline & & $\mathrm{CH} 270106$ & allinvalid & [if not missingPortDef] \\
\hline & & CH270107 & missingValue & \\
\hline \multirow[t]{8}{*}{ PA28 } & \multicolumn{4}{|c|}{ Elem_Port_Shdef_Unit } \\
\hline & \multicolumn{4}{|c|}{ CA2801 content of the set } \\
\hline & & CH280101 & deg & [if not missingPortDef] \\
\hline & & CH280102 & $\mathrm{rad}$ & [if not missingPortDef] \\
\hline & & $\mathrm{CH} 280103$ & combination & [if not (missingPortDef or onePortDef)] \\
\hline & & CH280104 & ContainInvalid & [if not (missingPortDef or onePortDef)] \\
\hline & & $\mathrm{CH} 280105$ & allinvalid & [if not missingPortDef] \\
\hline & & CH280106 & missingValue & \\
\hline \multirow[t]{9}{*}{ PA29 } & \multicolumn{4}{|c|}{ Elem Port_Scdef Val } \\
\hline & \multicolumn{4}{|c|}{ CA2901 content of the set } \\
\hline & & CH290101 & $>0$ & [if not missingPortDef] \\
\hline & & CH290102 & $=0$ & [if not missingPortDef] \\
\hline & & CH290103 & $<0$ & [if not missingPortDef] \\
\hline & & CH290104 & combination & [if not (missingPortDef or onePortDef)] \\
\hline & & CH290105 & ContainInvalid & [if not (missingPortDef or onePortDef)] \\
\hline & & CH290106 & allInvalid & [if not missingPortDef] \\
\hline & & CH290107 & missingValue & \\
\hline \multirow{7}{*}{ PA30 } & \multicolumn{4}{|c|}{ Elem Port Scdef Unit } \\
\hline & \multicolumn{4}{|c|}{ CA3001 content of the set } \\
\hline & & CH300101 & deg & [if not missingPortDef] \\
\hline & & CH300102 & $\mathrm{rad}$ & [if not missingPortDef] \\
\hline & & CH300103 & combination & [if not (missingPortDef or onePortDef)] \\
\hline & & CH300104 & ContainInvalid & [if not (missingPortDef or onePortDef)] \\
\hline & & CH300105 & allinvalid & [if not missingPortDef] \\
\hline \multirow[t]{9}{*}{ PA31 } & \multicolumn{4}{|c|}{ Elem Port Padef_Val } \\
\hline & \multicolumn{4}{|c|}{ CA3101 content of the set } \\
\hline & & CH310101 & $>0$ & [if not missingPortDef] \\
\hline & & CH310102 & $=0$ & [if not missingPortDef] \\
\hline & & $\mathrm{CH} 310103$ & $<0$ & [if not missingPortDef] \\
\hline & & CH310104 & combination & [if not (missingPortDef or onePortDef)] \\
\hline & & CH310105 & ContainInvalid & [if not (missingPortDef or onePortDef)] \\
\hline & & CH310106 & allinvalid & [if not missingPortDef] \\
\hline & & CH310107 & missingValue & \\
\hline \multirow[t]{7}{*}{ PA32 } & \multicolumn{4}{|c|}{ Elem_Port_Padef_Unit } \\
\hline & \multicolumn{4}{|c|}{ CA3201 content of the set } \\
\hline & & CH320101 & deg & [if not missingPortDef] \\
\hline & & CH320102 & $\mathrm{rad}$ & [if not missingPortDef] \\
\hline & & CH320103 & combination & [if not (missingPortDef or onePortDef)] \\
\hline & & CH320104 & ContainInvalid & [if not (missingPortDef or onePortDef)] \\
\hline & & CH320105 & allinvalid & [if not missingPortDef] \\
\hline \multirow[t]{9}{*}{ PA33 } & \multicolumn{4}{|c|}{ Grgeo_Add_Def } \\
\hline & CA3301 & Definition in & A \{HEXAGON, BLOC & JODE, POLYGON \\
\hline & & CH330101 & NODE & [property node] \\
\hline & & CH330102 & HEXAGON & [property hexagon] \\
\hline & & CH330103 & BLOCK & [property block] \\
\hline & & CH330104 & POLYGON & [property polygon] \\
\hline & CA3302 & Other value & & \\
\hline & & CH330201 & INVALID VALUE & [ERROR] \\
\hline & & CH330202 & errorMissing & [ERROR] \\
\hline
\end{tabular}




\begin{tabular}{|c|c|c|c|c|}
\hline \multicolumn{5}{|c|}{\begin{tabular}{|l|l|} 
PA34 & Grgeo Add Int Val1
\end{tabular}} \\
\hline & $\mathrm{CA3401}$ & integer & & \\
\hline & & CH340101 & $<0$ & [if node or hexagon or block or polygon] \\
\hline & & CH340102 & $=0$ & [if node or hexagon or block or polygon] \\
\hline & & CH340103 & $>0$ & [if node or hexagon or block or polygon] \\
\hline & CA3402 & Other value & & \\
\hline & & CH340201 & real number & [ERROR][if node or hexagon or block or polygon] \\
\hline & & CH340202 & non-number & [ERROR][if node or hexagon or block or polygon] \\
\hline & & $\mathrm{CH} 340203$ & errorMissing & [ERROR][if node or hexagon or block or polygon] \\
\hline \multirow[t]{9}{*}{ PA35 } & Grgeo_A & dd_Int_Val2 & & \\
\hline & CA3501 & integer & & \\
\hline & & $\mathrm{CH} 350101$ & $<0$ & [if node or hexagon or block or polygon] \\
\hline & & CH350102 & $=0$ & [if node or hexagon or block or polygon] \\
\hline & & CH350103 & $>0$ & [if node or hexagon or block or polygon] \\
\hline & CA3502 & Other value & & \\
\hline & & CH350201 & real number & [ERROR][if node or hexagon or block or polygon] \\
\hline & & CH350202 & non-number & [ERROR][if node or hexagon or block or polygon] \\
\hline & & CH350203 & errorMissing & [ERROR][if node or hexagon or block or polygon] \\
\hline \multirow[t]{10}{*}{ PA36 } & Grgeo_A & dd_Int_Val3 & & \\
\hline & CA3601 & integer & & \\
\hline & & CH360101 & $<0$ & [if block or polygon] \\
\hline & & CH360102 & $=0$ & [if block or polygon] \\
\hline & & CH360103 & $>0$ & [if block or polygon] \\
\hline & CA3602 & Other value & & \\
\hline & & CH360201 & real number & [ERROR][if block or polygon] \\
\hline & & CH360202 & non-number & [ERROR][if block or polygon] \\
\hline & & $\mathrm{CH} 360203$ & errorMissing & [ERROR][if block or polygon] \\
\hline & & CH360204 & missingValue & [if not(block or polygon)] \\
\hline \multirow[t]{10}{*}{ PA37 } & Grgeo_A & dd Int_Val4 & & \\
\hline & CA3701 & integer & & \\
\hline & & CH370101 & $<0$ & [if block or polygon] \\
\hline & & CH370102 & $=0$ & [if block or polygon] \\
\hline & & $\mathrm{CH} 370103$ & $>0$ & [if block or polygon] \\
\hline & CA3702 & Other value & & \\
\hline & & CH370201 & real number & [ERROR][if block or polygon] \\
\hline & & CH370202 & non-number & [ERROR][if block or polygon] \\
\hline & & CH370203 & errorMissing & [ERROR][if block or polygon] \\
\hline & & CH370204 & missingValue & [if not(block or polygon)] \\
\hline \multirow[t]{10}{*}{ PA38 } & Grgeo_A & dd Int Val5 & & \\
\hline & CA3801 & integer & & \\
\hline & & CH380101 & $<0$ & [if polygon] \\
\hline & & CH380102 & $=0$ & [if polygon] \\
\hline & & CH380103 & $>0$ & [if polygon] \\
\hline & CA3802 & Other value & & \\
\hline & & $\mathrm{CH} 380201$ & real number & [ERROR][if polygon] \\
\hline & & CH380202 & non-number & [ERROR][if polygon] \\
\hline & & CH380203 & errorMissing & [ERROR][if polygon] \\
\hline & & CH380204 & missingValue & [if not(polygon)] \\
\hline \multirow[t]{5}{*}{ PA39 } & Grgeo_A & dd_Int_Val6 & & \\
\hline & CA3901 & integer & & \\
\hline & & CH390101 & $<0$ & [if polygon] \\
\hline & & CH390102 & $=0$ & [if polygon] \\
\hline & & $\mathrm{CH} 390103$ & $>0$ & [if polygon] \\
\hline
\end{tabular}




\begin{tabular}{|c|c|c|c|c|}
\hline & CA3802 & Other value & & \\
\hline & & CH390201 & real number & [ERROR][if polygon] \\
\hline & & CH390202 & non-number & [ERROR][if polygon] \\
\hline & & CH390203 & errorMissing & [ERROR][if polygon] \\
\hline & & CH390204 & missingValue & [if not(polygon)] \\
\hline PA40 & Grgeo Ac & dd_Real_Val & & \\
\hline & CA4001 & real number & & \\
\hline & & CH400101 & $<0$ & [if node] \\
\hline & & CH400102 & $=0$ & [if node] \\
\hline & & CH400103 & $>0$ & [if node] \\
\hline & CA4002 & Other value & & \\
\hline & & CH400201 & non-number & [ERROR][if node] \\
\hline & & CH400202 & missingValue & \\
\hline PA41 & Grgeo Ar & dd Real Val & & \\
\hline & CA4101 & real number & & \\
\hline & & CH410101 & $<0$ & [if node] \\
\hline & & CH410102 & $=0$ & [if node] \\
\hline & & CH410103 & $>0$ & [if node] \\
\hline & CA4102 & Other value & & \\
\hline & & CH410201 & non-number & [ERROR][if node] \\
\hline & & CH410202 & missingValue & \\
\hline PA42 & Grgeo A & dd Real Val & & \\
\hline & CA4201 & real number & & \\
\hline & & CH420101 & $<0$ & [if node] \\
\hline & & CH420102 & $=0$ & [if node] \\
\hline & & CH420103 & $>0$ & [if node] \\
\hline & CA4202 & Other value & & \\
\hline & & CH420201 & non-number & [ERROR][if node] \\
\hline & & CH420202 & missingValue & \\
\hline PA43 & Grgeo_A & dd_Unit & & \\
\hline & CA4301 & Definition ir & a $\{\mathrm{deg}, \mathrm{rad}\}$ & \\
\hline & & CH430101 & deg & [if node] \\
\hline & & CH430102 & $\mathrm{rad}$ & [if node] \\
\hline & CA4302 & other value & & \\
\hline & & CH430201 & INVALID VALUE & [ERROR][if node] \\
\hline & & CH430202 & missingValue & \\
\hline PA44 & Grgeo A & dditional def & & \\
\hline & CA4401 & number of $s$ & et element & \\
\hline & & CH440101 & $=0$ & [property missingAddSet] \\
\hline & & CH440102 & $=1$ & [property singleAddSet] \\
\hline & & CH440103 & $>1$ & [property moreAddElem] \\
\hline & CA4402 & content of th & e set & \\
\hline & & CH440201 & NODE & [if singleAddSet] \\
\hline & & $\mathrm{CH} 440202$ & BLOCK & [if singleAddSet] \\
\hline & & CH440203 & HEXAGON & [if singleAddSet] \\
\hline & & CH440204 & combination & [if moreAddElem] \\
\hline & & CH440205 & missingValue & [if missingAddSet] \\
\hline PA45 & Grgeo A & dditional Int & Val1 & \\
\hline & CA4501 & content of $t$ & he set & \\
\hline & & CH450101 & $>0$ & [if not missingAddSet] \\
\hline & & CH450102 & $<0$ & [if not missingAddSet] \\
\hline & & CH450103 & $=0$ & [if not missingAddSet] \\
\hline & & CH450104 & combination & [if not missingAddSet] \\
\hline
\end{tabular}




\begin{tabular}{|c|c|c|c|c|}
\hline & & CH450105 & InvalidValue & [if not missingAddSet] \\
\hline & & CH450106 & missingValue & \\
\hline \multirow[t]{8}{*}{ PA46 } & \multirow{2}{*}{\multicolumn{4}{|c|}{ Grgeo_Additional Int Val2 }} \\
\hline & CA4601 & & & content of the set \\
\hline & & CH460101 & $>0$ & [if not missingAddSet] \\
\hline & & CH460102 & $<0$ & [if not missingAddSet] \\
\hline & & CH460103 & $=0$ & [if not missingAddSet] \\
\hline & & $\mathrm{CH} 460104$ & combination & [if not missingAddSet] \\
\hline & & CH460105 & InvalidValue & [if not missingAddSet] \\
\hline & & CH460106 & missingValue & \\
\hline \multirow[t]{8}{*}{ PA47 } & \multicolumn{4}{|c|}{ Grgeo_Additional Int_Val3 } \\
\hline & \multicolumn{4}{|c|}{ CA4701 content of the set } \\
\hline & & CH470101 & $>0$ & [if not missingAddSet] \\
\hline & & CH470102 & $<0$ & [if not missingAddSet] \\
\hline & & $\mathrm{CH} 470103$ & $=0$ & [if not missingAddSet] \\
\hline & & CH470104 & combination & [if not missingAddSet] \\
\hline & & CH470105 & InvalidValue & [if not missingAddSet] \\
\hline & & CH470106 & missingValue & \\
\hline \multirow[t]{8}{*}{ PA48 } & \multicolumn{4}{|c|}{ Grgeo_Additional Int Val4 } \\
\hline & \multicolumn{4}{|c|}{ CA4801 content of the set } \\
\hline & & CH480101 & $>0$ & [if not missingAddSet] \\
\hline & & CH480102 & $<0$ & [if not missingAddSet] \\
\hline & & CH480103 & $=0$ & [if not missingAddSet] \\
\hline & & CH480104 & combination & [if not missingAddSet] \\
\hline & & CH480105 & InvalidValue & [if not missingAddSet] \\
\hline & & CH480106 & missingValue & \\
\hline \multirow[t]{8}{*}{ PA49 } & \multirow{2}{*}{\multicolumn{4}{|c|}{ Grgeo Additional Int Val5 }} \\
\hline & & & & \begin{tabular}{|l|l|} 
CA4901 content of the set \\
\end{tabular} \\
\hline & & CH490101 & $>0$ & [if not missingAddSet] \\
\hline & & CH490102 & $<0$ & [if not missingAddSet] \\
\hline & & CH490103 & $=0$ & [if not missingAddSet] \\
\hline & & CH490104 & combination & [if not missingAddSet] \\
\hline & & CH490105 & InvalidValue & [if not missingAddSet] \\
\hline & & CH490106 & missingValue & \\
\hline \multirow[t]{8}{*}{ PA50 } & \multicolumn{4}{|c|}{ Grgeo_Additional_Int_Val6 } \\
\hline & \multicolumn{4}{|c|}{ CA5001 content of the set } \\
\hline & & CH500101 & $>0$ & [if not missingAddSet] \\
\hline & & CH500102 & $<0$ & [if not missingAddSet] \\
\hline & & CH500103 & $=0$ & [if not missingAddSet] \\
\hline & & CH500104 & combination & [if not missingAddSet] \\
\hline & & CH500105 & InvalidValue & [if not missingAddSet] \\
\hline & & CH500106 & missingValue & \\
\hline \multirow[t]{8}{*}{ PA51 } & \multicolumn{4}{|c|}{ Grgeo_Additional_Real_Val1 } \\
\hline & \multicolumn{4}{|c|}{\begin{tabular}{|l|l|} 
CA5101 & content of the set \\
\end{tabular}} \\
\hline & & CH510101 & $>0$ & [if not missingAddSet] \\
\hline & & CH510102 & $<0$ & [if not missingAddSet] \\
\hline & & CH510103 & $=0$ & [if not missingAddSet] \\
\hline & & CH510104 & combination & [if not missingAddSet] \\
\hline & & CH510105 & InvalidValue & [if not missingAddSet] \\
\hline & & CH510106 & missingValue & \\
\hline \multirow[t]{3}{*}{ PA52 } & \multicolumn{4}{|c|}{ Grgeo_Additional_Real_Val2 } \\
\hline & CA5201 & content of th & set & \\
\hline & & CH520101 & T>0 & [if not missingAddSet] \\
\hline
\end{tabular}




\begin{tabular}{|c|c|c|c|c|}
\hline & & CH520102 & $<0$ & [if not missingAddSet] \\
\hline & & CH520103 & $=0$ & [if not missingAddSet] \\
\hline & & CH520104 & combination & [if not missingAddSet] \\
\hline & & CH520105 & InvalidValue & [if not missingAddSet] \\
\hline & & CH520106 & missingValue & \\
\hline \multirow[t]{8}{*}{ PA53 } & \multicolumn{4}{|c|}{ Grgeo_Additional Real Val3 } \\
\hline & \multicolumn{4}{|c|}{ CA5301 content of the set } \\
\hline & & CH530101 & $>0$ & [if not missingAddSet] \\
\hline & & CH530102 & $<0$ & [if not missingAddSet] \\
\hline & & CH530103 & $=0$ & [if not missingAddSet] \\
\hline & & CH530104 & combination & [if not missingAddSet] \\
\hline & & CH530105 & InvalidValue & [if not missingAddSet] \\
\hline & & CH530106 & missingValue & \\
\hline \multirow[t]{7}{*}{ PA54 } & \multicolumn{4}{|c|}{ Grgeo_Additional_Unit } \\
\hline & \multicolumn{4}{|c|}{ CA5401 content of the set } \\
\hline & & CH540101 & deg & [if not missingAddSet] \\
\hline & & CH540102 & $\mathrm{rad}$ & [if not missingAddSet] \\
\hline & & CH540103 & combination & [if not missingAddSet] \\
\hline & & CH540104 & InvalidValue & [if not missingAddSet] \\
\hline & & CH540105 & missingValue & \\
\hline \multirow{6}{*}{ PA55 } & \multicolumn{4}{|c|}{ Group Grexc Def } \\
\hline & CA5501 & \multicolumn{3}{|c|}{ Definition in \{GROUP EXCITATION\} } \\
\hline & & CH550101 & GROUP EXCITATION & [property G_Excitation] \\
\hline & CA5502 & \multicolumn{3}{|l|}{ other value } \\
\hline & & CH550201 & INVALID VALUE & [ERROR] \\
\hline & & CH550202 & errorMissing & [ERROR] \\
\hline \multirow[t]{6}{*}{ PA56 } & \multicolumn{4}{|c|}{ Grexc_Gramp_Exec } \\
\hline & CA5601 & \multicolumn{3}{|c|}{ Definition in \{ AMPLITUDE \} } \\
\hline & & CH560101 & AMPLITUDE & [[property G Armplitude] \\
\hline & CA5602 & \multicolumn{3}{|l|}{ other value } \\
\hline & & CH560201 & INVALID VALUE & [ERROR] \\
\hline & & CH560202 & errorMissing & [ERROR] \\
\hline \multirow[t]{7}{*}{ PA57 } & \multicolumn{4}{|c|}{ Grexc_Gramp_Exec_Def } \\
\hline & CA5701 & \multicolumn{3}{|c|}{ Definition in \{UNIFORM, SECOND\} } \\
\hline & & CH570101 & UNIFORM & [if G_Amplitude, property gramp_uniform] \\
\hline & & CH570102 & SECOND & [if G_Amplitude, property gramp_second] \\
\hline & CA5702 & other value & & \\
\hline & & CH570201 & INVALID VALUE & [ERROR][if G_Amplitude] \\
\hline & & CH570202 & errorMissing & [ERROR][if G_Amplitude] \\
\hline \multirow[t]{9}{*}{ PA58 } & \multicolumn{4}{|c|}{ Grexc_Gramp_Exec_Vall } \\
\hline & CA5801 & \multicolumn{3}{|l|}{ real number } \\
\hline & & CH580101 & $=0$ & [if gramp_uniform or gramp_second] \\
\hline & & CH580102 & $>0$ & [if gramp_uniform or gramp_second] \\
\hline & & CH580103 & $<0$ & [if gramp uniform or gramp_second] \\
\hline & CA5802 & Other value & & \\
\hline & & CH580201 & non-number & [ERROR][if gramp_uniform or gramp_second] \\
\hline & & CH580202 & errorMissing & [ERROR][if gramp_second] \\
\hline & & CH580203 & missingValue & [if gramp uniform] \\
\hline PA59 & Grexc_G & ramp_Exec D & ef2 & \\
\hline & CA5901 & Definition in & $\{$ P1_VAL $\}$ & \\
\hline & & CH590101 & P1 VAL & [if gramp_second] \\
\hline & CA5902 & Other value & & \\
\hline & & CH590201 & INVALID VALUE & [ERROR][if gramp_second] \\
\hline
\end{tabular}




\begin{tabular}{|c|c|c|c|c|}
\hline & & CH590202 & errorMissing & [ERROR][if gramp second] \\
\hline & & CH590203 & missingValue & \\
\hline \multirow[t]{9}{*}{ PA60 } & \multicolumn{4}{|c|}{ Grexc_Gramp_Exec_Val2 } \\
\hline & CA6001 & \multicolumn{3}{|l|}{ real number } \\
\hline & & CH600101 & $=0$ & [if gramp second] \\
\hline & & CH600102 & $>0$ & [if gramp_second] \\
\hline & & CH600103 & $<0$ & [if gramp second] \\
\hline & CA6002 & \multicolumn{3}{|l|}{ Other value } \\
\hline & & CH600201 & non-number & [ERROR][if gramp second] \\
\hline & & CH600202 & errorMissing & [ERROR][if gramp second] \\
\hline & & CH600203 & missingValue & \\
\hline \multirow[t]{7}{*}{ PA61 } & \multicolumn{4}{|c|}{ Grexc_Gramp_Exec_Def3 } \\
\hline & CA6101 & \multicolumn{3}{|c|}{ Definition in $\{$ P2 VAL $\}$} \\
\hline & & CH610101 & P2 VAL & [if gramp_second] \\
\hline & CA6102 & \multicolumn{3}{|l|}{ Other value } \\
\hline & & $\mathrm{CH} 610201$ & INVALID VALUE & [ERROR][if gramp_second] \\
\hline & & CH610202 & errorMissing & [ERROR][if gramp second] \\
\hline & & CH610203 & missingValue & \\
\hline \multirow[t]{9}{*}{ PA62 } & \multicolumn{4}{|c|}{ Grexc_Gramp_Exec_Val3 } \\
\hline & CA6201 & \multicolumn{3}{|l|}{ real number } \\
\hline & & CH620101 & $=0$ & [if gramp_second] \\
\hline & & CH620102 & $>0$ & [if gramp_second] \\
\hline & & CH620103 & $<0$ & [if gramp_second] \\
\hline & CA6202 & \multicolumn{3}{|l|}{ Other value } \\
\hline & & CH620201 & non-number & [ERROR][if gramp second] \\
\hline & & CH620202 & errorMissing & [ERROR][if gramp_second] \\
\hline & & CH620203 & missingValue & \\
\hline \multirow[t]{7}{*}{ PA63 } & \multicolumn{4}{|c|}{ Grexc_Gramp_Exec_Def4 } \\
\hline & CA6301 & \multicolumn{3}{|c|}{ Definition in $\{\mathrm{Q} 1 \mathrm{VAL}\}$} \\
\hline & & CH630101 & Q1 VAL & [if gramp_second] \\
\hline & CA6302 & \multicolumn{3}{|c|}{ Other value } \\
\hline & & CH630201 & INVALID VALUE & [ERROR][if gramp_second] \\
\hline & & CH630202 & errorMissing & [ERROR][if gramp_second] \\
\hline & & CH630203 & missingValue & \\
\hline \multirow[t]{9}{*}{ PA64 } & \multicolumn{4}{|c|}{ Grexc_Gramp_Exec Val4 } \\
\hline & CA6401 & \multicolumn{3}{|l|}{ real number } \\
\hline & & CH640101 & $=0$ & [if gramp second] \\
\hline & & CH640102 & $>0$ & [if gramp second] \\
\hline & & CH640103 & $<0$ & [if gramp_second] \\
\hline & CA6402 & \multicolumn{3}{|l|}{ Other value } \\
\hline & & CH640201 & non-number & [ERROR][if gramp_second] \\
\hline & & CH640202 & errorMissing & [ERROR][if gramp_second] \\
\hline & & CH640203 & missingValue & \\
\hline PA65 & Grexc G & ramp Exec I & Def5 & \\
\hline & CA6501 & Definition in & $n\{Q 2-V A L\}$ & \\
\hline & & CH650101 & Q2 VAL & [if gramp_second] \\
\hline & CA6502 & Other value & & \\
\hline & & CH650201 & INVALID VALUE & [if gramp_second] \\
\hline & & CH650202 & missingValue & \\
\hline PA66 & Grexc_G & ramp Exec & Val5 & \\
\hline & CA6601 & real number & & \\
\hline & & CH660101 & $=0$ & [if gramp_second] \\
\hline & & CH660102 & $>0$ & [if gramp_second] \\
\hline
\end{tabular}




\begin{tabular}{|c|c|c|c|c|}
\hline & & $\mathrm{CH} 660103$ & $<0$ & [if gramp_second] \\
\hline & CA6602 & \multicolumn{3}{|l|}{ Other value } \\
\hline & & CH660201 & non-number & [ERROR][if gramp second] \\
\hline & & CH660202 & errorMissing & [ERROR][if gramp second] \\
\hline & & CH660203 & missingValue & \\
\hline \multirow[t]{12}{*}{ A67 } & \multicolumn{4}{|c|}{ Grexc Gramp_Exec Unit } \\
\hline & CA6701 & \multicolumn{3}{|c|}{ Definition in $\{\mathrm{m}, \mathrm{mm}, \mathrm{dm}, \mathrm{cm}$ LINEAR, POWER, $\mathrm{dB}\}$} \\
\hline & & CH670101 & LINEAR & [if gramp uniform] \\
\hline & & CH670102 & POWER & [if gramp uniform] \\
\hline & & CH670103 & $\mathrm{dB}$ & [if gramp_uniform] \\
\hline & & CH670104 & $\mathrm{mm}$ & [if gramp_second] \\
\hline & & CH670105 & $\mathrm{dm}$ & [if gramp_second] \\
\hline & & CH670106 & $\mathbf{c m}$ & [if gramp_second] \\
\hline & & CH670107 & $\mathbf{m}$ & [if gramp_second] \\
\hline & CA6702 & Other value & & \\
\hline & & CH670201 & INVALID VALUE & [ERROR][if gramp uniform or gramp second] \\
\hline & & CH670202 & missingValue & \\
\hline \multirow{5}{*}{ PA68 } & \multicolumn{4}{|c|}{ Grexc Grpha Exec } \\
\hline & CA6801 & \multicolumn{3}{|c|}{ Definition in \{ PHASE \} } \\
\hline & & CH680101 & PHASE & [property phase] \\
\hline & CA6802 & \multicolumn{3}{|l|}{ other value } \\
\hline & & CH680201 & INVALID_VALUE & [ERROR] \\
\hline \multirow[t]{8}{*}{ PA69 } & \multicolumn{4}{|c|}{ Grexc_Grpha Def } \\
\hline & CA6901 & \multicolumn{3}{|c|}{ Definition in \{ UNIFORM, SECOND, ROTATION, POINTING \} } \\
\hline & & CH690101 & UNIFORM & [property grpha_uniform] \\
\hline & & CH690102 & SECOND & [property grpha second] \\
\hline & & CH690103 & ROTATION & [property grpha rotation] \\
\hline & & CH690104 & POINTING & [property grpha_pointing] \\
\hline & CA6902 & \multicolumn{3}{|c|}{ other value } \\
\hline & & \multicolumn{3}{|c|}{\begin{tabular}{|l|l|} 
CH690201 & INVALID VALUE \\
\end{tabular}} \\
\hline \multirow[t]{9}{*}{ PA70 } & \multicolumn{4}{|c|}{ Grexc_Grpha_Exec_Def } \\
\hline & CA7001 & \multicolumn{3}{|c|}{ Definition in $\{\mathrm{CW}, \mathrm{CCW}, \mathrm{U}, \mathrm{THETA}\}$} \\
\hline & & CH700101 & CW & [if grpha rotation] \\
\hline & & CH700102 & $\mathrm{CCW}$ & [if grpha_rotation] \\
\hline & & CH700103 & $\mathbf{U}$ & [if grpha pointing, property pointing_axis] \\
\hline & & $\overline{\mathrm{CH} 700104}$ & THETA & [if grpha pointing, property pointing angle] \\
\hline & CA7002 & \multicolumn{3}{|l|}{ other value } \\
\hline & & CH700201 & INVALID_VALUE & [ERROR] \\
\hline & & CH700202 & missingValue & \\
\hline PA71 & \multicolumn{4}{|c|}{ Grexc_Grpha_Exec Vall } \\
\hline & CA7101 & \multicolumn{3}{|c|}{ values in[-1.1] } \\
\hline & & CH710101 & $=0$ & [if not grpha uniform] \\
\hline & & CH710102 & $=-1$ & [if pointing_axis] \\
\hline & & $\mathrm{CH} 710103$ & $=1$ & [if pointing_axis] \\
\hline & & CH710104 & $(-1,0)$ & [if pointing axis] \\
\hline & & CH710105 & $(0,1)$ & [if pointing_axis] \\
\hline & CA7102 & \multicolumn{3}{|l|}{ real number } \\
\hline & & CH710201 & $>0$ & [if not (grpha_uniform or pointing_axis)] \\
\hline & & CH710202 & $<0$ & [if not (grpha_uniform or pointing axis)] \\
\hline & CA7103 & other value & & \\
\hline & & CH710301 & $<-1$ & [ERROR][if pointing_axis] \\
\hline & & CH710302 & $>1$ & [ERROR][if pointing_axis] \\
\hline & & CH710303 & non-number & [ERROR][if not grpha_uniform] \\
\hline
\end{tabular}




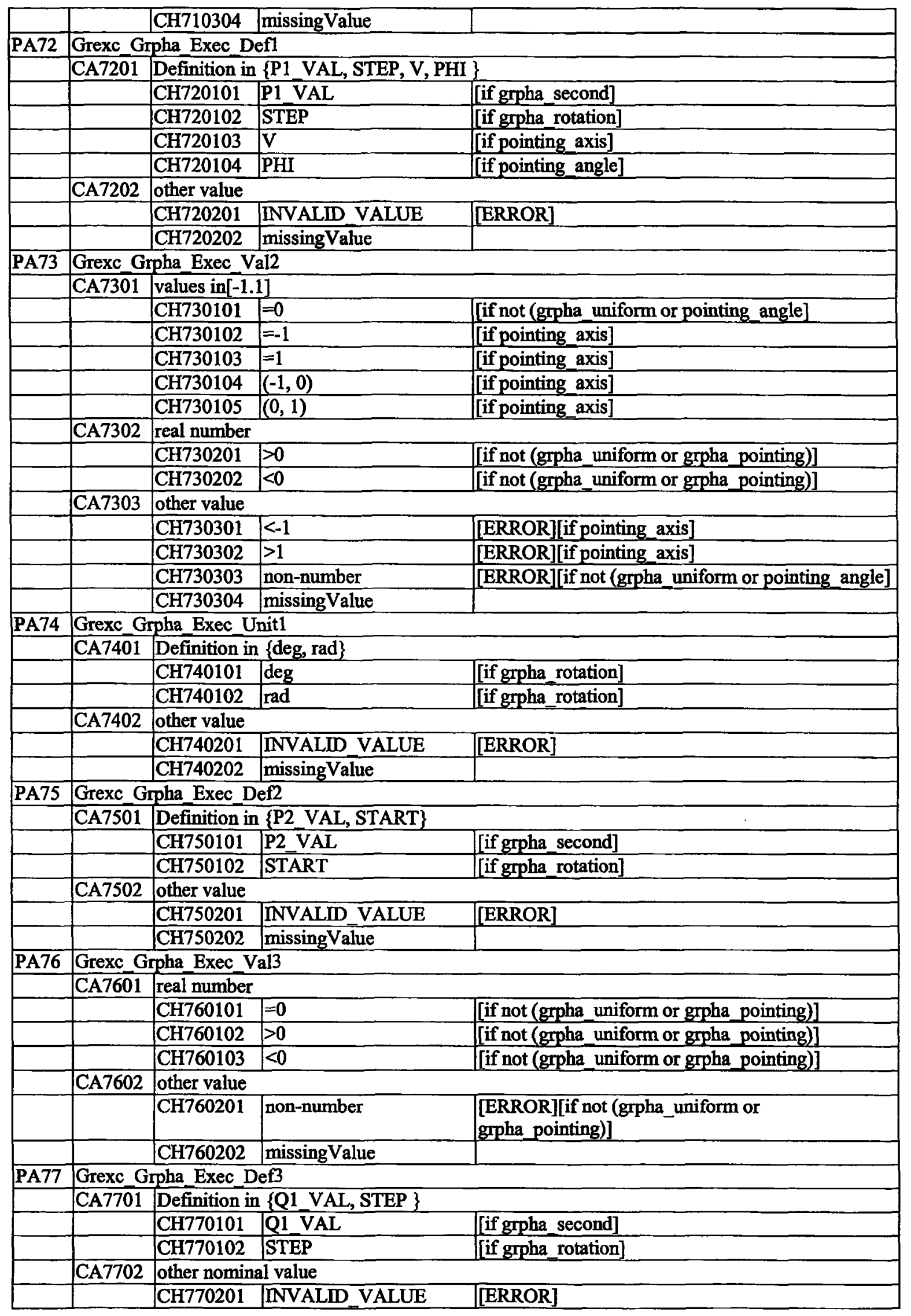




\begin{tabular}{|c|c|c|c|c|}
\hline & \multicolumn{3}{|c|}{\begin{tabular}{|l|l|} 
CH770202 & missingValue \\
\end{tabular}} & \\
\hline \multirow[t]{8}{*}{ PA78 } & \multicolumn{4}{|c|}{ Grexc_Grpha_Exec_Val4 } \\
\hline & CA7801 & \multicolumn{3}{|l|}{ real number } \\
\hline & & CH780101 & $=0$ & $\begin{array}{l}\text { [if not (grpha_uniform or grpha_rotation or } \\
\text { grpha_pointing)] }\end{array}$ \\
\hline & & $\mathrm{CH} 780102$ & $>0$ & $\begin{array}{l}\text { [if not (grpha_uniform or grpha_rotation or } \\
\text { grpha_pointing)] }\end{array}$ \\
\hline & & $\mathrm{CH} 780103$ & $<0$ & $\begin{array}{l}\text { [if not (grpha_uniform or grpha_rotation or } \\
\text { grpha_pointing)] }\end{array}$ \\
\hline & CA7802 & other value & & \\
\hline & & $\mathrm{CH} 780201$ & non-number & $\begin{array}{l}\text { [ERROR][if not (grpha_uniform or grpha_rotation } \\
\text { or grpha_pointing)] }\end{array}$ \\
\hline & & CH780202 & missingValue & \\
\hline \multirow[t]{6}{*}{ PA79 } & \multicolumn{4}{|c|}{ Grexc Grpha Exec Def4 } \\
\hline & CA7901 & \multicolumn{3}{|c|}{ Definition in $\{\mathrm{Q} 2 \mathrm{VAL}\}$} \\
\hline & & CH790101 & Q2 VAL & [if grpha_second] \\
\hline & CA7902 & \multicolumn{3}{|l|}{ other value } \\
\hline & & CH790201 & INVALID VALUE & [ERROR] \\
\hline & & \multicolumn{3}{|c|}{\begin{tabular}{|l|l} 
CH790202 & missingValue \\
\end{tabular}} \\
\hline \multirow[t]{8}{*}{ PA80 } & \multicolumn{4}{|c|}{ Grexc_Grpha_Exec_Val5 } \\
\hline & CA8001 & \multicolumn{3}{|l|}{ real number } \\
\hline & & CH800101 & $=0$ & [if not pointing_axis] \\
\hline & & CH800102 & $>0$ & [if not pointing_axis] \\
\hline & & CH800103 & $<0$ & [if not pointing axis] \\
\hline & CA8002 & \multicolumn{3}{|l|}{ other value } \\
\hline & & CH800201 & non-number & [ERROR][if not pointing axis] \\
\hline & & CH800202 & missingValue & \\
\hline \multirow[t]{7}{*}{ PA81 } & \multicolumn{4}{|c|}{ Grexc_Grpha_Exec_Unit2 } \\
\hline & CA8101 & \multicolumn{3}{|c|}{ Definition in $\{\mathrm{deg}, \mathrm{rad}\}$} \\
\hline & & CH810101 & deg & [if not pointing_axis] \\
\hline & & CH810102 & $\mathrm{rad}$ & [if not pointing_axis] \\
\hline & CA8102 & \multicolumn{3}{|l|}{ other value } \\
\hline & & CH810201 & |INVALID VALUE & [ERROR] \\
\hline & & CH810202 & missingValue & \\
\hline PA82 & \multicolumn{4}{|c|}{ Recursive Def } \\
\hline & CA8201 & \multicolumn{3}{|c|}{$\bar{D}$ Definition in \{NONE, TRIANG, POLAR, POL ORIENT, PHASE\} } \\
\hline & & CH820101 & NONE & \\
\hline & & CH820102 & TRIANG & \\
\hline & & CH820103 & POLAR & \\
\hline & & CH820104 & POL_ORIENT & \\
\hline & & CH820105 & PHASE & \\
\hline & CA8202 & \multicolumn{3}{|l|}{ other value } \\
\hline & & CH820201 & INVALID VALUE & [ERROR] \\
\hline & & CH820202 & missingValue & \\
\hline PA83 & \multicolumn{4}{|c|}{ Test_Result } \\
\hline & CA8301 & \multicolumn{3}{|c|}{ Test result of the case } \\
\hline & & CH830101 & $\mathbf{P}$ & \\
\hline & & CH830102 & $\mathbf{F}$ & \\
\hline
\end{tabular}




\section{C.3 Detailed analysis result}

\section{C.3.1 Strategy one: diagnose all statements executed}
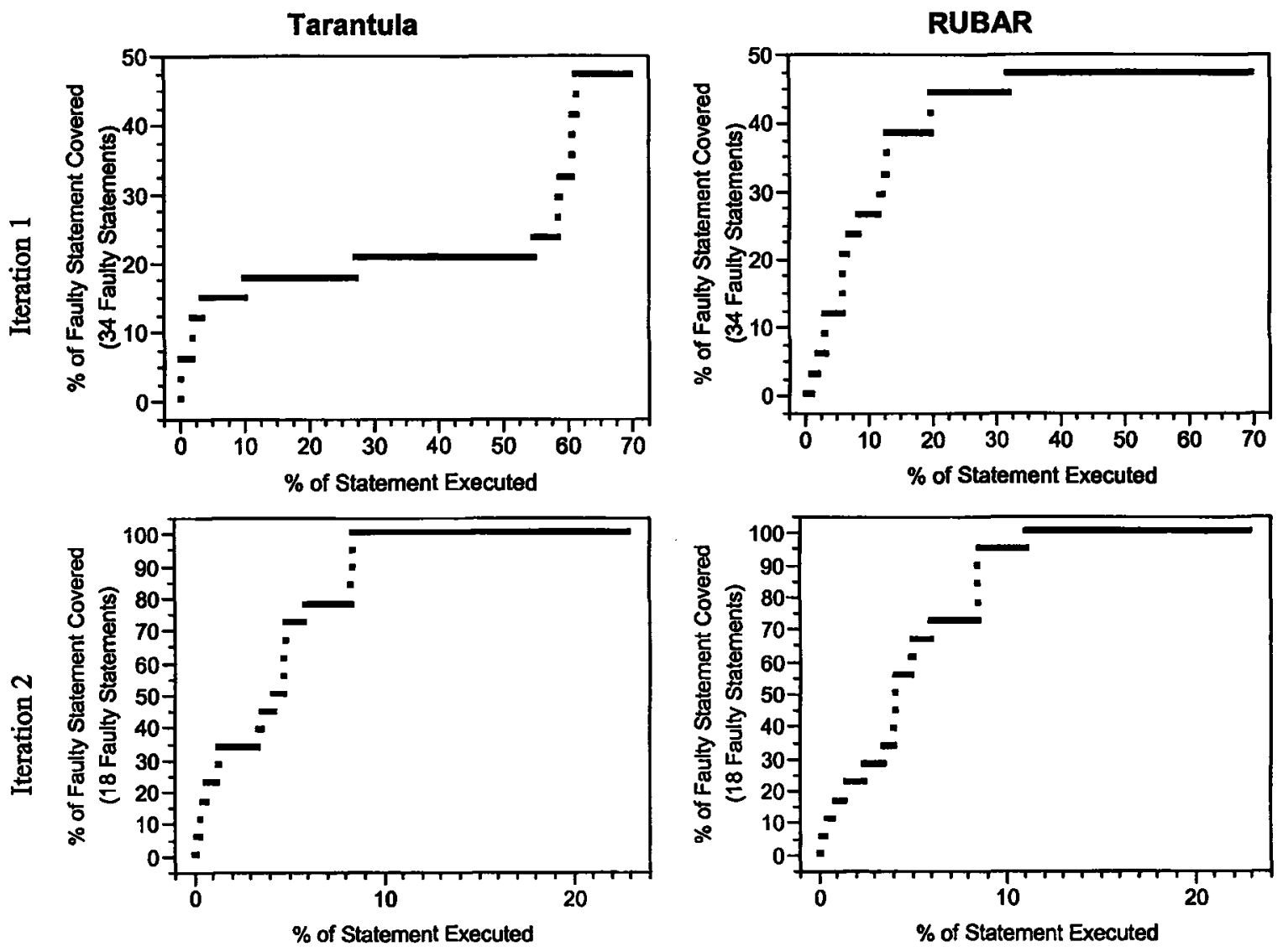

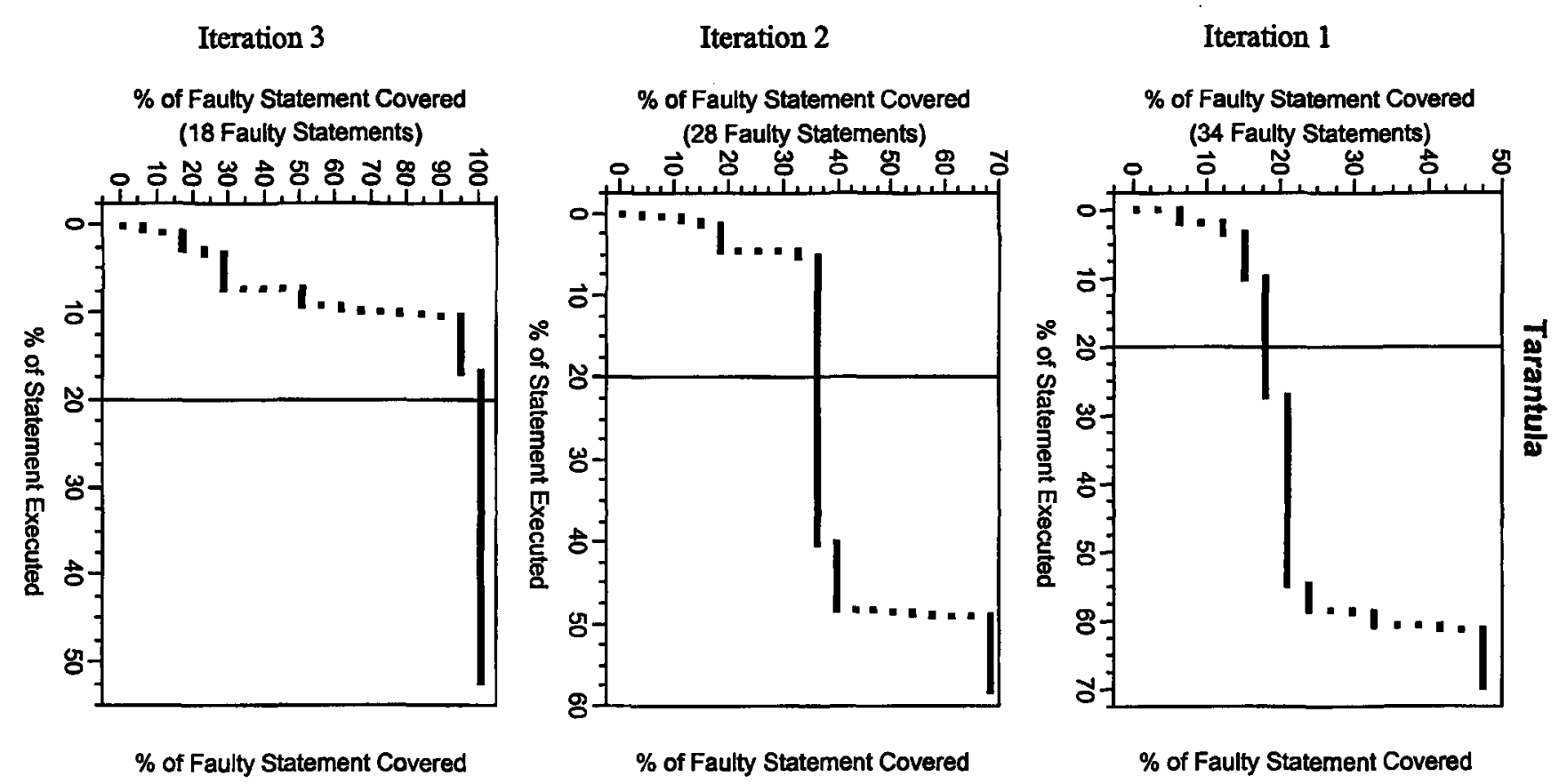

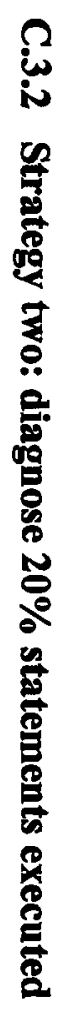
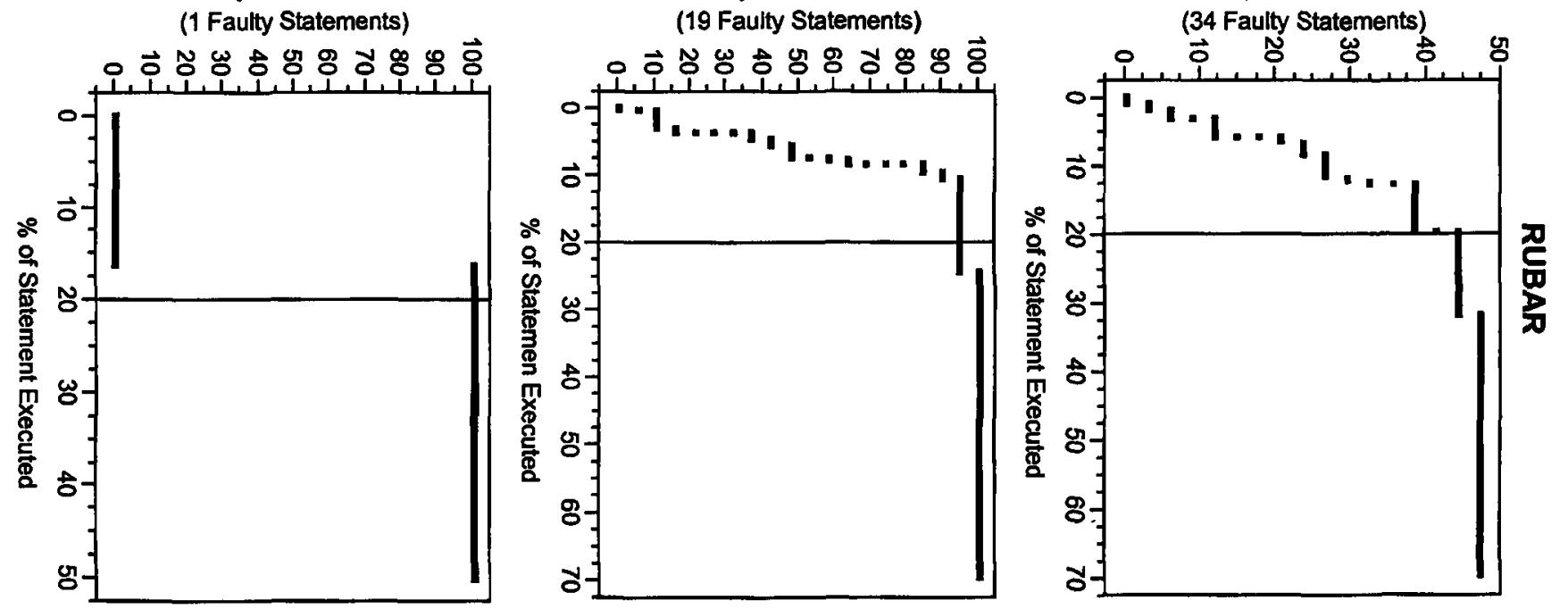


\section{APPENDIX D THE PACKHEXCHAR PROGRAM}

\section{D.1 Sample C4.5 Trees}

\section{D.1.1 Ver-71}

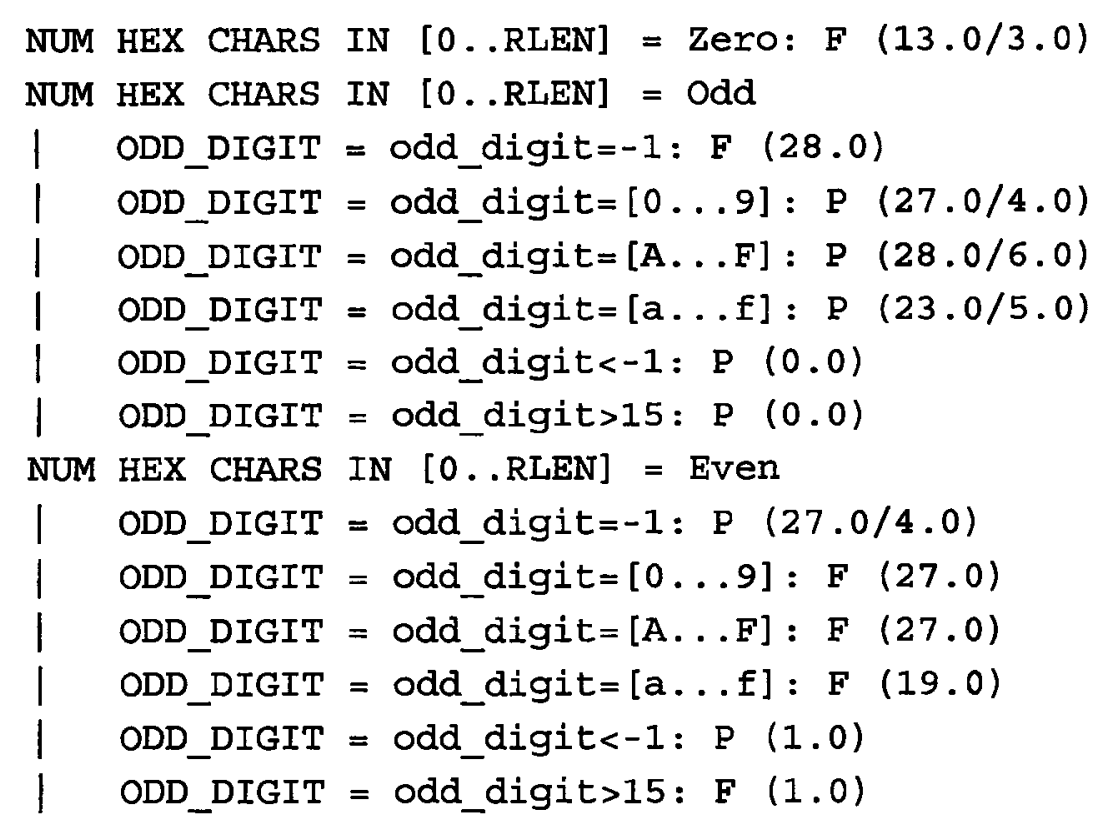

Number of Leaves : 13

Size of the tree : 16

$===$ Stratified cross-validation $===$

Correctly Classified Instances 19

Incorrectly Classified Instances 23

Kappa statistic

$89.5928 \%$

Mean absolute error

0.7899

0.1729

Root mean squared error

0.3034

Relative absolute error

$35.8075 \%$

Root relative squared error

Total Number of Instances

$61.751 \%$

221

$===$ Detailed Accuracy By Class ===

$\begin{array}{rrrrrrc}\text { TP Rate } & \text { FP Rate } & \text { Precision } & \text { Recall } & \text { F-Measure } & \text { ROC Area } & \text { Class } \\ 0.956 & 0.145 & 0.819 & 0.956 & 0.882 & 0.889 & \text { P } \\ 0.855 & 0.044 & 0.966 & 0.855 & 0.907 & 0.889 & \text { F }\end{array}$


$===$ Confusion Matrix $===$

a b <-- classified as

\begin{tabular}{ll|l}
86 & 4 & $\mathrm{a}=\mathrm{P}$
\end{tabular}

$19112 \mid \mathrm{b}=\mathrm{F}$

\section{D.1.2 Ver-4872}

CHAR CASE $=N / A: P(13.0)$

CHAR CASE = allNumbers: P $(52.0)$

CHAR CASE = allLowerCase: $\mathrm{F}(52.0)$

CHAR CASE = allCaps: $P(52.0)$

CHAR CASE $=$ mixedCase $: F(52.0 / 3.0)$

Number of Leaves : 5

size of the tree : 6

$===$ Stratified cross-validation $===$

Correctly Classified Instances 218

Incorrectly Classified Instances

3

0.9727

Kappa statistic

Mean absolute error

Root mean squared error

Relative absolute error

Root relative squared error

Total Number of Instances
0.0259

0.1165

$5.227 \%$

$23.3818 \%$

221

$===$ Detailed Accuracy By Class ===

$\begin{array}{ccccrrr}\text { TP Rate } & \text { FP Rate } & \text { Precision } & \text { Recall } & \text { F-Measure } & \text { ROC Area } & \text { Class } \\ 0.975 & 0 & 1 & 0.975 & 0.987 & 0.989 & \text { P } \\ 1 & 0.025 & 0.971 & 1 & 0.985 & 0.989 & \text { F }\end{array}$

$===$ Confusion Matrix $===$

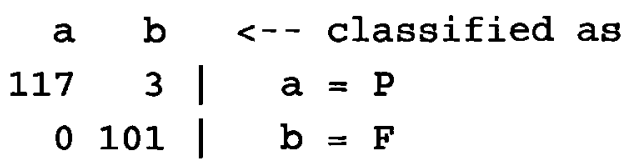

\section{D.1.3 Ver-13849}

CHAR CASE $=$ N/A: P $(13.0)$

CHAR CASE $=$ allNumbers

$$
\begin{aligned}
& \text { ODD_DIGIT }=\text { odd_digit }=-1: \text { F }(12.0) \\
& \text { ODD_DIGIT }=\text { odd_digit }=[0 \ldots 9]: \text { P }(14.0) \\
& \text { ODD_DIGIT }=\text { odd_digit }=[\text { A } \ldots F]: P(14.0) \\
& \text { ODD_DIGIT }=\text { odd_digit }=[\text { a } \ldots \text { f }]: P(12.0)
\end{aligned}
$$




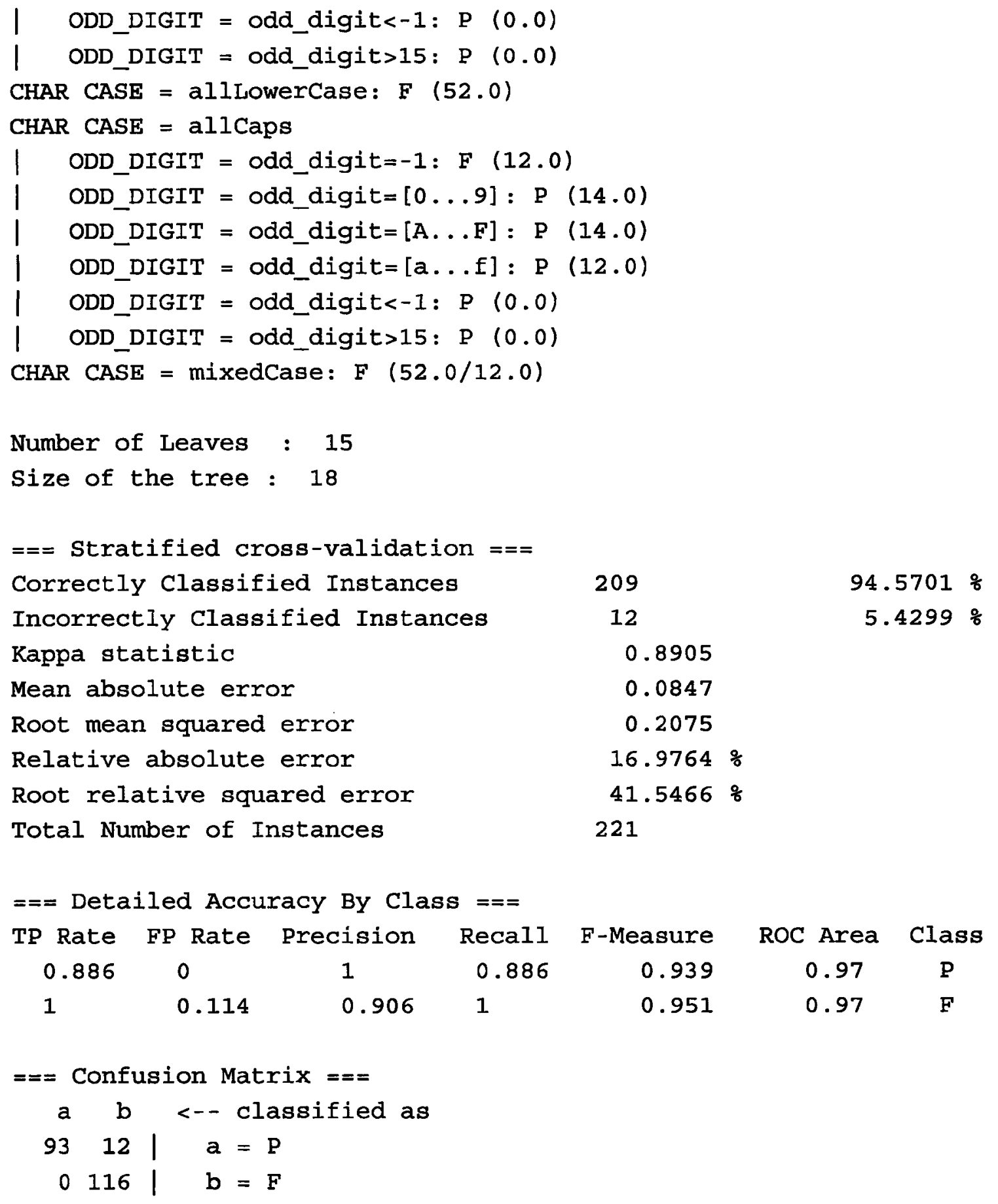

